\title{
Thermal Oscillations in Sea Ice and Soils
}

by

Nitay Ben Shachar

\author{
A thesis \\ submitted to the Victoria University of Wellington \\ in fulfilment of the \\ requirements for the degree of \\ Master of Science \\ in Mathematics. \\ Victoria University of Wellington





\begin{abstract}
We present mathematical analysis of temperature oscillations in depth-dependent media by investigating the thermodynamics of sea ice and of soils. Time-series temperature measurements from thermistor strings are common in both sea ice and soils and are used to study their properties, evolution, seepage flux and a host of interactions with their environment. We use numerical tools and perturbation theory to study the propagation of high frequency, small amplitude temperature oscillations through the in-homogeneous media using one dimensional models. Analytical tools for studying such thermal waves are derived.

In sea ice the absorption of solar radiation and oscillating air temperatures result in two distinct thermal wave propagation behaviours. At depths, stationary waves associated with in place solar heating are observed, whereas near the surface, travelling thermal waves are present due to the quick decay in the absorbed solar radiation and the oscillatory air temperatures. These are observed in thermistor string data taken in McMurdo Sound, Antarctica between 1996-2003. Using a variety of mathematical tools, the leading order behaviour of the diurnal temperature oscillation is approximated in terms of elementary functions and is compared with results from numerical simulations.

The thermodynamics of soils differ from sea ice in that all the solar radiation is absorbed at the upper boundary and water movement within the soil carries heat. Macroscale in-homogeneity in the advection-diffusion equation is considered and the thermal wave propagation characteristics are studied using a WKB approximation. The leading order behaviour is shown to reduce exactly to the Stallman equations, being the solution to the thermal wave propagation in a homogeneous soil with constant, uniform water flow. We use the leading order WKB expansion to estimate errors in the homogeneous soil assumption commonly made to estimate the seepage velocity and soil diffusivity. It is shown that the diffusivity estimations are relatively stable and provide reasonably accurate results, but the seepage velocity estimations incur significant errors that should be considered. A frequency dependence in the error leads us to suggest multi-frequency analysis for detection and further studies of the effects of in-homogeneous soil thermodynamics.
\end{abstract}




\section{Acknowledgments}

The support I have received during the thesis year far exceeds anything I could summarize in a few paragraphs, although every bit of it has been instrumental in making this project as fun and educational as it was. First and foremost thank you to my supervisors, Mark McGuinness and Joe Trodahl, who provided me with guidance, expertise, and valuable teachings throughout the year. To Mark, thank you for your wise advice and careful guidance. It made it a pleasure to study and learn. Thank you also for the valuable feedback and proofreading of the thesis. And to Joe, thank you for your patience, expertise, and resilience throughout the year, our chats never failed to steer me to understand details and take care where necessary. I would also like to thank Inga Smith and Shaun Eaves for allowing me to attend and present at the NZ Sea Ice Symposium and the SIRG Annual Meeting. These experiences were instrumental for me to understand the breadth of work being done in the field.

Thank you also to my friends and cohort for their support, a special thank you to Linus Richter, Malcolm Jones, Ellen Hammatt, Thomas Berry and Lilian Stott. 


\section{Contents}

Abstract i

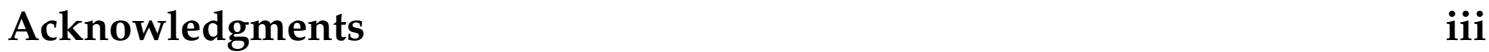

List of Figures $\quad$ x

List of Tables $\quad$ xi

\begin{tabular}{lll}
\hline & Introduction & 1
\end{tabular}

1.1 Importance of Sea Ice . . . . . . . . . . . . . . . . . . . . 1

1.1 .1 Climate. . . . . . . . . . . . . . . . 2

1.1 .2 Ecological Importance . . . . . . . . . . . . . . . . . . . . 2

1.2 Structure and the Growth Process of Sea Ice . . . . . . . . . . . . . . 3

1.3 Periodic Forcing . . . . . . . . . . . . . . . . . 5

1.4 Soils and Sediments . . . . . . . . . . . . . . . . 7

1.5 Outline of Thesis . . . . . . . . . . . . . . . 8

\begin{tabular}{|lll}
2 & Transient Temperature Simulator for Sea Ice & 11
\end{tabular}

2.1 Background . . . . . . . . . . . . . . . . 11

2.2 Sea Ice Thermodynamics . . . . . . . . . . . . . . . . . . . . . . 13

2.3 Discretization and Freezing the Boundary . . . . . . . . . . . . . . 16

$2.4 \quad$ Validating the Model . . . . . . . . . . . . . . . . . . . 19

$2.4 .1 \quad$ Ice Thickness Estimation . . . . . . . . . . . . . . . . . . . . 19

2.4 .2 Estimating Oceanic Heat Flux . . . . . . . . . . . . . . 20

2.4 .3 Numerical Simulation of Ice Temperatures . . . . . . . . . . 20

2.5 Further Application: Snow Cover . . . . . . . . . . . . . . . . 26

2.6 Summary . . . . . . . . . . . . . . . . 27

3 Oscillatory Temperatures in Sea Ice 29

3.1 Background . . . . . . . . . . . . . . . . 30 
3.2 Differential Equation for Thermal Waves . . . . . . . . . . . 34

3.2 .1 Parameters. . . . . . . . . . . . . . . . . . . . . . . . . 39

3.2 .2 Oscillatory Air Temperatures . . . . . . . . . . . . . . . . 41

3.2 .3 Non-constant Conductivity Terms . . . . . . . . . . . . . . 43

3.3 Numerical and Data Comparison . . . . . . . . . . . . . . . . 44

4 Mathematical Approximations 49

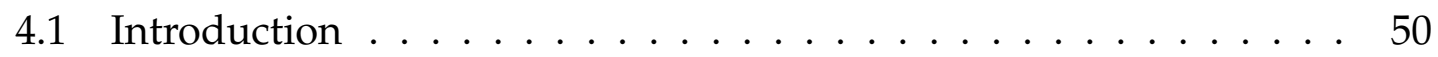

4.1 .1 Matched Asymptotics $\ldots \ldots \ldots$. . . . . . . . . 50

$4.1 .2 \quad$ WKB Theory $\ldots \ldots \ldots \ldots \ldots$. . . . . . . . . . 50

4.1 .3 Laplace's Method . . . . . . . . . . . . . . . . . . . . . 52

4.1 .4 Method of Steepest Descent . . . . . . . . . . . . . . . . 53

4.2 Boundary Layer Analysis $\ldots \ldots \ldots \ldots \ldots$

4.2 .1 Matched Asymptotics . . . . . . . . . . . . . . . . . . 54

4.2 .2 Interpretations and Numerical Comparisons . . . . . . . 56

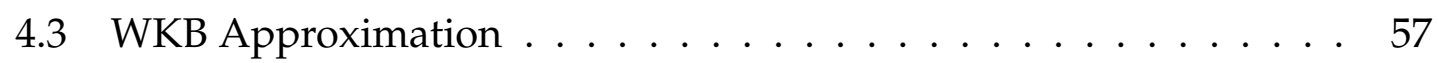

4.3 .1 Homogeneous System . . . . . . . . . . . . . . . . 60

4.3 .2 Non-Homogeneous Case - Distributed Source . . . . . . . 63

$4.3 .3 \quad$ Asymptotic form in the limit $\delta \rightarrow 0 \ldots \ldots \ldots \ldots$. . . . . 69

4.4 Extensions to any Time-Dependent Diurnal Source. . . . . . . . . 79

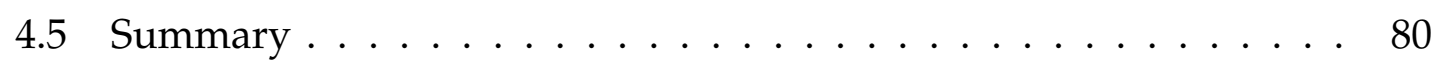

$\begin{array}{llr}5 & \text { Soil Thermodynamics } & 81\end{array}$

5.1 Introduction . . . . . . . . . . . . . . . . . . . . . . 82

5.1.1 Temperature Time Series Analysis Methods. . . . . . . . . 82

5.1 .2 Data Analysis . . . . . . . . . . . . . . . . . 83

5.1 .3 Non-Uniform Soils . . . . . . . . . . . . . . . . . . . . . . 84

5.2 Differential Equation for Thermal Waves in Soils . . . . . . . . . 86

5.2 .1 General Approximate Solutions . . . . . . . . . . . . . 91

5.2.2 Reduction to Homogeneous Soils with Constant, Uniform Seepage Velocity . . . . . . . . . . . . . . . 93

5.3 Numerical Validation . . . . . . . . . . . . . . . . . . . . . . 93

5.4 In-Homogeneous Soils with No Seepage . . . . . . . . . . . . . 97

5.5 In-Homogeneous Soils with Seepage $\ldots \ldots \ldots \ldots \ldots$

$5.5 .1 \quad$ Numerical Examples . . . . . . . . . . . . . . . . . . . . . 98

5.6 Case Study - Linear Thermal Conductivity . . . . . . . . . . . . . 99

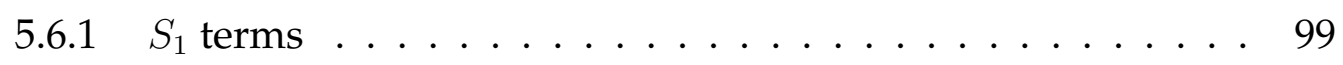

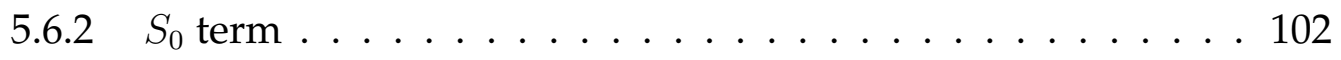


5.6 .3 Errors in $\kappa_{e, \text { measured }}$ and $\xi_{\text {measured }} \ldots \ldots$. . . . . . . 104

\begin{tabular}{lll}
\hline & Conclusions & 107
\end{tabular}

\begin{tabular}{lr}
\hline Bibliography & 122
\end{tabular}

\begin{tabular}{|ll}
\hline A Physical Parameters and Constants & 123
\end{tabular}

A.1 Ice Properties . . . . . . . . . . . . . . . . . . . . . . 123

A.2 Snow Properties . . . . . . . . . . . . . . . . . . . . . . . . 124

A.3 Common Soil Properties . . . . . . . . . . . . . . . . . . . . 124 
$4.1 \quad$ Inner and outer solutions compared with numerical solutions . . . 58

4.2 Inner and outer solutions compared with numerical solutions . . . 59

4.3 Homogeneous WKB approximation and numerical solutions . . . . 64

4.4 WKB approximation and numerical solutions . . . . . . . . . . . . . . . 67

4.5 WKB approximation and numerical solutions . . . . . . . . . . . . . 68

$4.6 \quad$ Real and imaginary components of the phase function, $\phi(s)$. . . 72

$4.7 \quad$ Elementary function approximation of the WKB approximation . . 77

4.8 Elementary function approximation of the WKB approximation . . 78

5.1 Soil thermistor string measurements . . . . . . . . . . . . . . . 85

5.2 Amplitude decay and phase shift of the diurnal signal . . . . . . . . 86

5.3 Amplitude decay and phase shift vs frequency . . . . . . . . . . . . . . 87

$5.4 \quad$ Estimations of $\kappa$ and $v_{t}$ vs frequency $\ldots \ldots \ldots$. . . . . . . . 88

5.5 Numerical simulation of the advection-diffusion equation . . . . . 94

5.6 Numerical simulations and WKB approximation vs depth . . . . . 95

$5.7 \quad$ WKB approximation vs frequency for linearly depth varying and constant thermal conductivity . . . . . . . . . . . . . . . 100

5.8 real and imaginary parts of $S_{1}$ term in the WKB approximation . . 101

5.9 Error ration of $S_{0}$ term for an average thermal conductivity as-

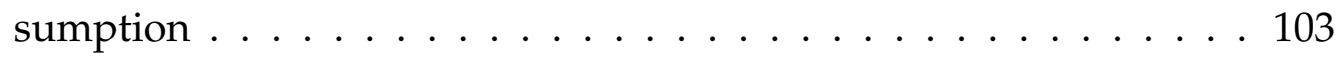

$5.10 \kappa$ and $v_{t}$ estimates from numerical simulations WKB approximation 106 


\section{List of Tables}

$3.1 \quad$ Parameters of distributed heating source term in sea ice . . . . . . . 41

3.2 Small parameter, $\delta$, values . . . . . . . . . . . . . 42 


\section{Chapter 1}

\section{Introduction}

Sea ice is formed by the freezing of sea water in the colder months, typically in high latitude regions, and forms one of the largest annual cycle on the planet. For example, Antarctic sea ice extent ranges from 19 million $\mathrm{km}^{2}$ to 3 million $\mathrm{km}^{2}$ at its extremes [16]. Sea ice extent has been studied thoroughly with the aid of satellite imagery [82] but models fail to capture the observed decline in sea ice extent [106]. Sea ice models form key components in forecasting the global climate and its response to perturbations in the climate.

A large fraction of the sea ice is first year sea ice which grows in the colder months to a thickness of up to about $2 \mathrm{~m}$ and melts in the warmer months. Some sea ice survives the warmer months to become second year or multi year ice. The properties of the multi year ice differ from that of first year ice due to its age and history, such as its density and thermal properties [75, 100]. Further, a snow cover on the ice can become flooded to form layers of sea ice with varying properties as the snow properties themselves are highly dynamic due to compression, redistribution by winds and meltpond formation.

\subsection{Importance of Sea Ice}

Sea ice plays a key factor in the global climate dynamics and is an indicator of climate change. Further, sea ice harbours ecosystems and its presence and absence plays a key role in the life cycle of these organisms. Hence studying sea ice is of great importance, as will be discussed in the following sub-sections. 


\subsubsection{Climate}

The properties of sea ice are extremely important for climate modelling as sea ice forms a physical, optical and thermodynamic barrier between the ocean and the atmosphere, mediating the heat exchange between the two [97]. Sea ice is inherently coupled to the ocean and atmosphere due to its interactions with them. For example, the energy budget of the ocean is highly dependent on the amount of sun light entering the ocean. A thin layer of sea ice reflects a significant portion of the incoming radiation back into the atmosphere [55], and hence sea ice extent, depth, surface albedo and many other factors will dictate the solar energy flux into the ocean. The albedo effect has the greatest implications on the global climate and has been the first term to be incorporated in global models, for which sea ice was simply included by 'painting the sea white'. Further effects include the insulation sea ice provides between the ocean and the atmosphere, brine rejection from freezing sea ice into the ocean affecting ocean circulation, heat storage and release in the freezing/melting of the sea ice into the ocean, sea ice breakup and movement and many more.

It is important to note that the effects of sea ice on the global climate form both positive and negative feedback loops. The albedo effect forms a positive feedback loop, where greater growth of sea ice leads to less solar radiation absorbed in high latitude regions and so more sea ice growth can occur. The thermal barrier that sea ice forms constitutes a negative feedback loop, where more sea ice cover insulates the oceans, reducing the heat loss and the amount of sea ice that can form.

The thermodynamic interactions between sea ice, atmosphere and the ocean are inherently linked to the physical properties and physical interactions that occur, and vice versa. Ice extent is regulated by ice freeze in dates, ice breakups, ridges and all other ice dynamics, all of which play a crucial role in coupling to the ice cover and its effects. As will be discussed later, the physical and thermal properties of sea ice are inherently linked due to its complex structure.

\subsubsection{Ecological Importance}

Algae and other biological organisms get trapped in the freezing sea ice. The vertical distribution of the algae depends on the growth rate of the sea ice and the currents in the water column below. The presence of sea ice forms a physical boundary for organisms to live on, and can protect the organisms from lethal UV radiation, however thick sea ice may block radiation necessary for photosyn- 
thesis [72]. During the melt of sea ice organic material is released into the water column, with some fish migrating underneath melting sea ice to make use of the increased food availability. Studying sea ice behaviour is necessary to develop our understanding of the interactions between these organisms and their unique habitat.

\subsection{Structure and the Growth Process of Sea Ice}

Sea ice is a complex material due to the inclusion of brine as well as other impurities. Fundamentally, it is modelled as a mushy layer [21,36]; a solid matrix of ice, bathed in liquid water with impurities (brine). Brine is a patch of highly saline sea water, the salt being rejected by nearby freezing ice crystals. Brine has significantly different thermal properties to pure ice and hence the composite material of the two has varying and dynamic thermal and physical properties. Brine pockets expand and contract with temperature as well as migrating through the sea ice [56]. These brine pockets can become elongated and connect and form brine tubes which when connected to the ocean flush out carrying heat and salts, as well as other impurities with them [75]. The dynamic nature of the brine pockets and tubes adds a dynamic aspect to the physical and thermal properties of sea ice. Sea ice is inherently a complex 3 dimensional substance due to these brine pockets and impurities. However, with increasing measurements, accurate bulk thermal and physical properties have been calculated and account for brine and other impurities.

The presence of brine pockets and other impurities induce complex optical interactions in the sea ice via scattering and absorption [19,69]. The optical properties of sea ice are necessary to understand the sea ice's effects on the oceanatmosphere interaction and further the thermodynamics that occur in the sea ice.

Around Antarctica, sea ice initially freezes by forming frazil ice, many small ice crystals sloshed by waves, currents and winds. These are stirred and mixed until a layer with thickness of around $20-50 \mathrm{~cm}$ of frazil ice crystals is present on the surface, at which point the ice is able to solidify together and form a slab. After a solid ice slab is achieved, further heat conduction allows crystals with their fastest growth directed vertically to dominate the growth process, and relatively clear, columnar ice is formed [103]. The top layer of frazil ice comprising randomly oriented crystals has distinctively different optical properties to the columnar ice grown at deeper depths.

Super-cooled water currents near ice shelves lead to platelet ice crystals to 
form in the supercooled water. These are buoyant and float up to the ice slab, sticking to the bottom of the ice. As the columnar ice grows further the platelet crystals get incorporated into the ice. These have been observed extensively in locations such as McMurdo Sound, Antarctica and can contribute significantly to the ice thickness [95]. The platelet crystals can be observed in crystal cross sections from ice cores under cross-polarized filters [103].

As well as affecting the optical and thermodynamic properties of sea ice, the mechanical properties of sea ice yield interesting ice dynamics. Sea ice dynamics can result in both sea ice breakup and growth. Ocean waves have been shown to be a good predictor for sea ice breakup in certain regions of the Antarctic [50], as well as other factors such as ocean currents and winds. The effects of these interactions are mediated by the thickness of the sea ice [50,67]. An accurate understanding of the breakup of sea ice is therefore reliant on the prediction of sea ice thickness, a poorly understood and hard to measure quantity. Further, the inclusion of the brine tubes and pockets in the sea ice compromises its mechanical strength when a temperature of about $-5{ }^{\circ} \mathrm{C}[23]$ is reached. At this temperature the brine tubes get large enough to connect and flush into the ocean, allowing for warm ocean water to rise up within the sea ice. The sea ice turns into a slush and melts rapidly.

From the above discussion we see that the thermodynamics and dynamics of sea ice are inherently linked. Better understandings of both is necessary to inform large scale models and predict the effects that various forcing factors will have on sea ice behaviour.

Forcing factors are the conditions imposed on the sea ice and snow. For example, the air temperatures near the surface of the sea ice/snow, the amount of radiation falling on the sea ice/snow and the ocean swells and currents. Inherently all these processes are linked together with the sea ice and affect one another. For modelling purposes, a model for each component (such as a sea ice model, ocean model, atmospheric model etc.) is usually simulated independently with forcing factors being parameterized; simulating the entire climate together and its intricate inter-dependencies is not practical with the computational tools available. A great emphasis has been put on analysing measurements to get accurate parameterizations of prominent forcing factors. For example, projects such as SHEBA (Surface HEat Budget of the Arctic Ocean) [105] have aimed to parameterize the sea ice albedo and more with measurements. This sea ice - radiation interaction has been linked to varying amounts of sea ice melt [107] and internal puddle formation [1, 43] with measurements of the radiation characteristics being essential 
for such studies [109].

\subsection{Periodic Forcing}

Understanding the effects of periodic driving forces on sea ice is of great importance due to the periodic nature of air temperatures, solar irradiance, ocean currents, tides and many more natural processes that interact with the sea ice. Resolving the details of daily variations is important for accurate predictions to be made, even within one season [27]. The two periodic interactions we will focus on are the air temperature and the short wave solar radiation penetrating the sea ice and snow. The periodic air temperatures dictate the boundary condition (the energy balance at the surface of the sea ice), whereas the solar irradiance affects the energy balance within the sea ice.

Thermistor string measurements taken near McMurdo Sound, Antarctica show daily temperature oscillations, as has been examined by [66, 103] and as can be seen in Figure 1.1. The daily oscillations are of smaller amplitude and faster frequency to the amplitude and frequency of the sea ice's thermal response to weather systems (occurring over weeks and months).

The thermodynamic effects of oscillatory boundary conditions on sea ice have been studied by [108], but without the availability of good parameterizations of the physical and thermal properties the analysis was limited. An estimation for the thermal conductivity of sea ice was attained by analysis of measured thermal wave propagation, however, it was shown that solar radiation, being unaccounted for rigorously, complicated the calculations. The analysis of the thermal wave propagation required the removal of the effect of the solar radiation absorption which was done via trial and error fittings or by analysing data where insignificant solar radiation was present. Based on this work and that of [53], a more comprehensive model was developed by [18], predicting the temperature field of a homogeneous translucent medium and refining estimates of the thermal diffusivity by [53] of an in-homogeneous medium when temperature and heat flux measurements are available. More recently, numerical analysis of the solar heating of sea ice, ice sheets, snow and other semi-transparant media have become widely used [5, 19, 66, 92, 101]. Heat propagation through porous, fibrous or in general an-isotropic and in-homogeneous materials has become an interest point due to their technological applications, amongst these are functionally graded materials [98]. In Chapters 3 and 4 we provide an analytical approximation for the propagation behaviour of thermal waves in sea ice due to solar heat- 

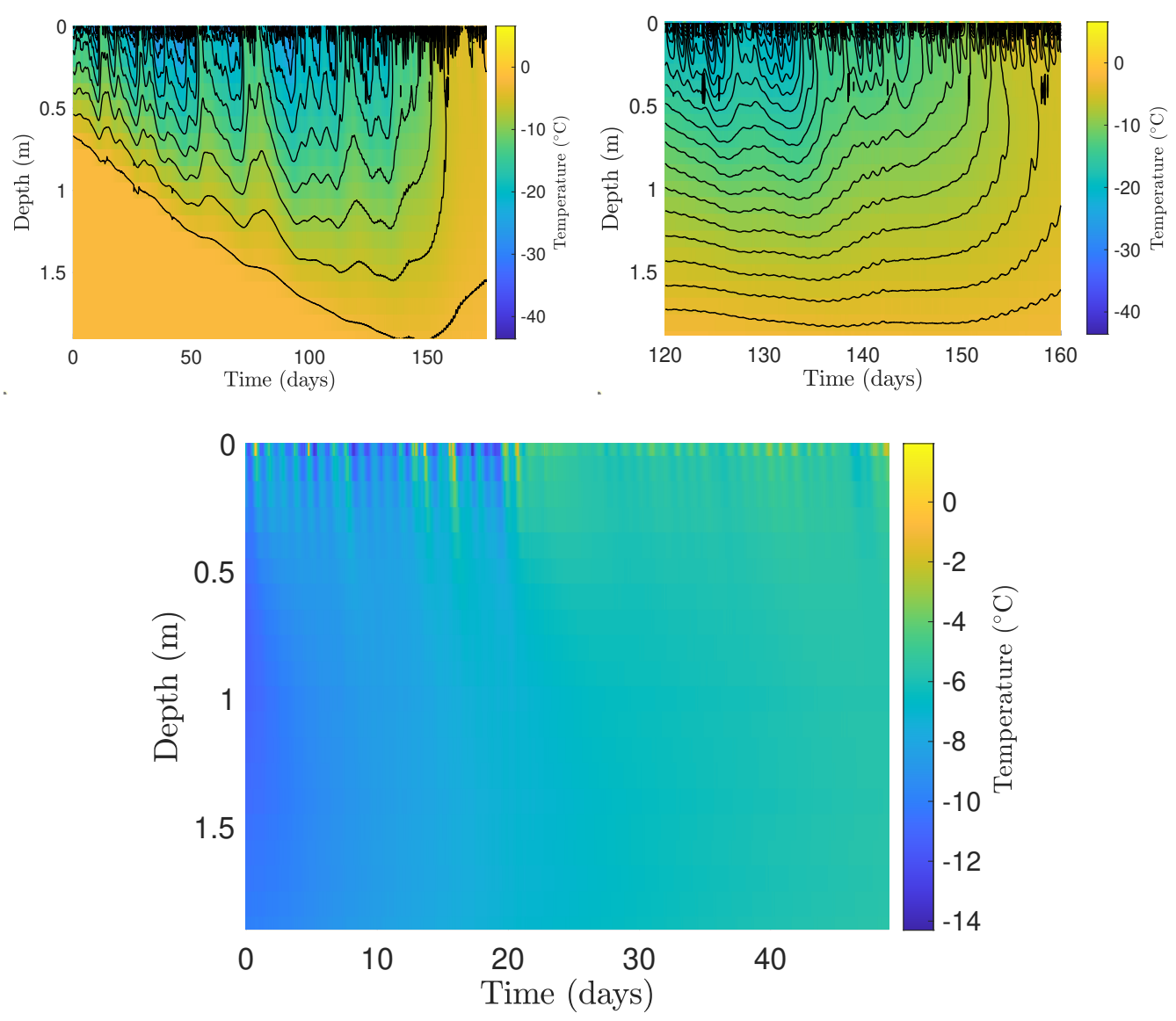

Figure 1.1: Thermistor string data from first year Antarctic sea ice 1996 (top) and multiyear Antarctic sea ice 2003 (bottom). The colours indicate temperature in ${ }^{\circ} \mathrm{C}$ according to the colour bars to the right of each plot. The top left plot shows the 1996 measurements over the entire range that they were taken and on the right the same plot is cropped slightly with further contours added to highlight the daily oscillations present in the data. The thermistors were deployed (day 0 in the plots) on the 12th of June, 1996 and 7th of November, 2003. We see the amplitude of the daily oscillations is much smaller and oscillates much faster than the weather systems. The thermistor string deployed in 2003 into multiyear sea ice exhibits strong daily oscillations too, with a storm dumping about $20 \mathrm{~cm}$ of snow on the ice after $\approx 20$ days from the beginning of the measurement as can be seen by the sudden temperature rise. 
ing and small amplitude oscillations in the air temperature following the work presented in [66] and further build on it with asymptotic analysis.

\subsection{Soils and Sediments}

In addition to studying sea ice thermodynamics, a project to study soil thermodynamics was undertaken. We provide an introduction to soil thermodynamics below and present the remainder of the work associated with this project in Chapter 5 .

The study of soil and sediment thermodynamics is of great importance to various fields of study such as hydrology and geology, with applications in building science, agriculture and ecology. Most notably, soil thermodynamics are used in large scale modelling for IPCC reports in the study of climate change [97]. However, it is also found in studies estimating groundwater movement, river seepage, soil characteristics and Earth-air heat exchangers. It has become common to use heat as a tracer for groundwater movement due to the water's movement carrying heat. Further, temperature measurements are relatively cheap and easy and do not pollute the environment like chemical tracers. Although heat pulse injection may be used to make such measurements, many authors have opted for using natural heat as a tracer in the form of temperature measurements. Common analysis methods track the amplitude and phase of various temperature oscillations as they propagate into the soil. For these methods careful measurements, analysis and theory for the amplitude and phase propagation of a thermal wave must be undertaken.

Soils are modelled as a heterogeneous, porous medium composed of a solid matrix of various compounds with fluid flow occurring between the pores (both water vapour). Soils and sediments are typically composed of various materials with varying grain sizes and structures. Intricate models must be undertaken in order to determine the bulk thermal properties of such a medium. The study of the effects of soil heterogeneity ranges on scales of microscopic (the sizes of the pores) to macroscopic, such as various sediment layers or impurity inclusions. Following [80], early suggestions included estimating the bulk thermal conductivity of a two phase medium by the geometric mean of a two phases weighted by their volume fraction. Theoretical models bound the bulk thermal conductivity [29] of such materials. Homogenization of the heat equation [31] indicates that the microscopic variations may be averaged over in the leading order behaviour of the thermal waves, although the average taken may not be the 
average expected. For example, the effective thermal conductivity is calculated as $k_{\text {ave }}=\left\langle k^{-1}\right\rangle^{-1}$ where $<$. $>$ indicates averaging over the small scale dependence. The homogenized thermodynamics model used for soils is the advectiondiffusion equation [2,39].

$$
\rho c \frac{\partial T}{\partial t}=\frac{\partial}{\partial z}\left(k \frac{\partial T}{\partial z}\right)-q \rho_{w} c_{w} \frac{\partial T}{\partial z}
$$

where $\rho$ and $c$ are the bulk density and specific heat capacity of the soil, $\rho_{w}$ and $c_{w}$ are the density and specific heat capacity of water, $q$ is the vertical seepage velocity (water flow) and $k$ is the effective thermal conductivity. Exact solutions to this equation were derived by [96] in the case of a homogeneous soil with a constant, uniform seepage velocity, with most analytical and numerical tools using these assumptions [30, 44, 62, 65]. However, there is a growing interest in analysing the effects of heterogeneous soils [48, 86], non-constant [81] and nonuniform [68] seepage fluxes as well as other theory assumptions [41], all of which have only been studied numerically.

\subsection{Outline of Thesis}

The remainder of this thesis is presented as follows. A numerical simulator of sea ice transient temperatures is presented in Chapter 2 and validated against a number of sea ice thermistor strings. The simulator is designed to aid in the studies presented in the remainder of the thesis as well as being an easy to use tool for further sea ice thermodynamics research. This material will be submitted shortly to the Journal of Oceanic and Atmospheric Technology. In Chapter 3 we introduce a two time analysis to derive a differential equation for the leading order behaviour of quick, small amplitude thermal waves due to solar heating and oscillating air temperatures. Data analysis is presented along with numerical solutions and simulation results. In Chapter 4 we use boundary layer theory to construct the leading order behaviour of solutions to the thermal waves in sea ice using matched asymptotics and a WKB approximation and further approximate these in terms of elementary functions using the method of steepest descent. Chapter 5 contains the soil thermodynamics project, in which we analyse the effects of the heterogeneity of soils on the resulting thermal wave propagation using perturbation methods. This is used to derive errors in common tools used to infer soil diffusivity and seepage fluxes which are based on thermal waves in 
homogeneous soils. Conclusions of the two projects are summarised in Chapter 6 as well as possible future work. 


\section{Chapter 2}

\section{Transient Temperature Simulator for Sea Ice}

\subsection{Background}

Many thermistor string measurements have been taken of sea ice temperature, with data repositories available online, such as in [71]. Some successes of the thermistor string projects include an improved parameterization of the thermal conductivity of sea ice [77], detection of supercooled water currents beneath the sea ice [95], tracking snow and ice depths / thickness over time [54], parameterizing the albedo [105] and many more. To further analyse such data, comparisons between the data and numerical modelling aid in quantifying contributions of various parameters and processes. For example, simulations with various oceanic flux's or salinity profiles can be compared to each other and the data set.

A rigorous model of the sea ice thermodynamics is no easy task due to the multi-phase nature of sea ice and its complicated, three dimensional structure (see section 1.2. . Mushy layer models of sea ice [21,36] have indicated that during the growth phase the sea ice is well modelled by neglecting horizontal heat transfer and assuming the thermodynamics are represented by the bulk properties of sea ice, which have been measured extensively (see Appendix A). This allows for the development of a much simpler model of sea ice thermodynamics being a one dimensional, non-linear heat equation. Numerical models for studying the temperature transients in sea ice in this fashion have been developed [51, 63, 83, 99], each being geared towards various applications such as multi-year modelling of multi-year ice, slush layer inclusion, first year sea ice growth or the inclusion of biological species, with great success. Numerical schemes are used where discretizations in time and space allows for a numerical approximation of the non- 


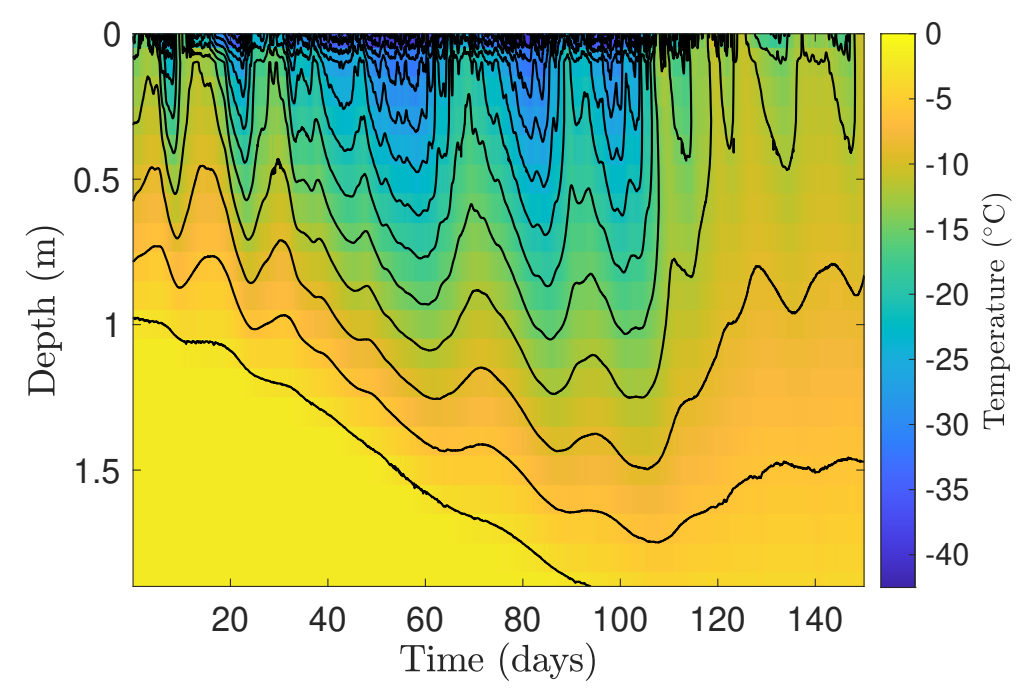

Figure 2.1: Thermistor string measurements of first year sea ice from McMurdo Sound, Antarctica in the winter months of 1997.

linear heat equation to be iterated, coupled with the selected boundary conditions.

A drawback of some previous models is the expensive computations required for transient temperature calculations, hindering the coupling of such models to large scale climate models where they must be evaluated on many grid cells. There has been an emphasis on developing suitable, lightweight models that would couple well with full climactic models [35, 110]. The lightweight models must be verified against rigorous models and data to ensure the assumptions made do not lead to significant biases developing. For example, studies have found that significant errors develop when diurnal (daily) cycles of forcing data is neglected [27].

In this chapter we develop in MATLAB the existing one-dimensional thermodynamics model discussed in section 2.2 for the growth of sea ice and compare it to thermistor string data. Features such as ice depth and snow thickness have been analysed by previous models and are recreated well [54]. The numerical solver will be used to analyse the finer details present in the thermistor string data, such as the temperature transients and ice growth rate. This numerical tool will aid in analysing the thermal waves in response to the solar radiation absorption in later chapters, as well as further numerical studies of sea ice. 


\subsection{Sea Ice Thermodynamics}

The heat equation has been studied thoroughly in various applications [14, 28, 40]. The solutions can only be expressed in terms of elementary functions in very specific formulations of the equation. For this reason it is very common to study the solutions numerically [15, 20,34,51,110, 114] especially when comparing to sea ice temperature data.

We consider the processes that take place after stable sea ice has formed, and assume a one dimensional model following [51,63]. The model is not designed to simulate the melting of sea ice, or the formation of slushy layers at the snow /ice interface.

Let the thickness of the ice be given by $H(t)$ and the thickness of the snow cover be given by $h(t)$, as illustrated in Figure 2.2. We assume a sharp interface between the ice and the ocean, meaning that the ice/ocean interface is at $z=$ $-H(t) . z=0$ is set to be the ice/snow interface. At all times we have $H(t) \leq 0$ and $h(t) \geq 0$. Inside the ice we would like to satisfy the heat equation,

$$
\rho c \frac{\partial T}{\partial t}=\frac{\partial}{\partial z}\left(k \frac{\partial T}{\partial z}\right)+P(z, t), z \in[-H, 0],
$$

where $\rho$ is the density, $c$ is the heat capacity, $k$ is the thermal conductivity of sea ice and $P(z, t)$ is the power absorbed per $m^{3}$ due to penetrating short wave radiation. The absorbed power is parameterized as an exponential decay in three regions (in the snow, the upper $20 \mathrm{~cm}$ of the sea ice and the remainder of the sea ice), $P(z, t)=P_{0}(t) A e^{-\alpha z}$, with a constant extinction coefficient, $\alpha$, in each region. The respective extinction coefficients used are 20, 20 and $2 \mathrm{~m}^{-1}$ following [55, 58, 69, 73]. $P(z, t)$ is ensured to be continuous across the boundaries of these regions with relevant prefactors, $A$, in each region. Hence, $A$ and $\alpha$ are piecwise constant functions of depth. The amount of solar radiation entering the ice/snow top layer, $P_{0}$, is given by [91] as below. Other parameterizations of incident power and cloud cover parameterization such as detailed in [46] may be adopted.

$$
P_{0}=(1-\alpha)(1-0.52 c) S_{0} \frac{\cos ^{2}(Z)}{1.2 \cos (Z)+10^{-3} V(2.7+\cos (Z))+0.1},
$$

where $V$ is the vapour pressure, $\alpha$ is the albedo, $Z$ is the solar zenith angle, $S_{0}$ is the solar constant of $1362 \mathrm{~W} / \mathrm{m}^{2}$ and $c$ is a cloudiness factor employed by [10]. The cloudiness factor is provided by the user while the solar zenith angle is computed using the package provided in [47] and the user specified lati- 

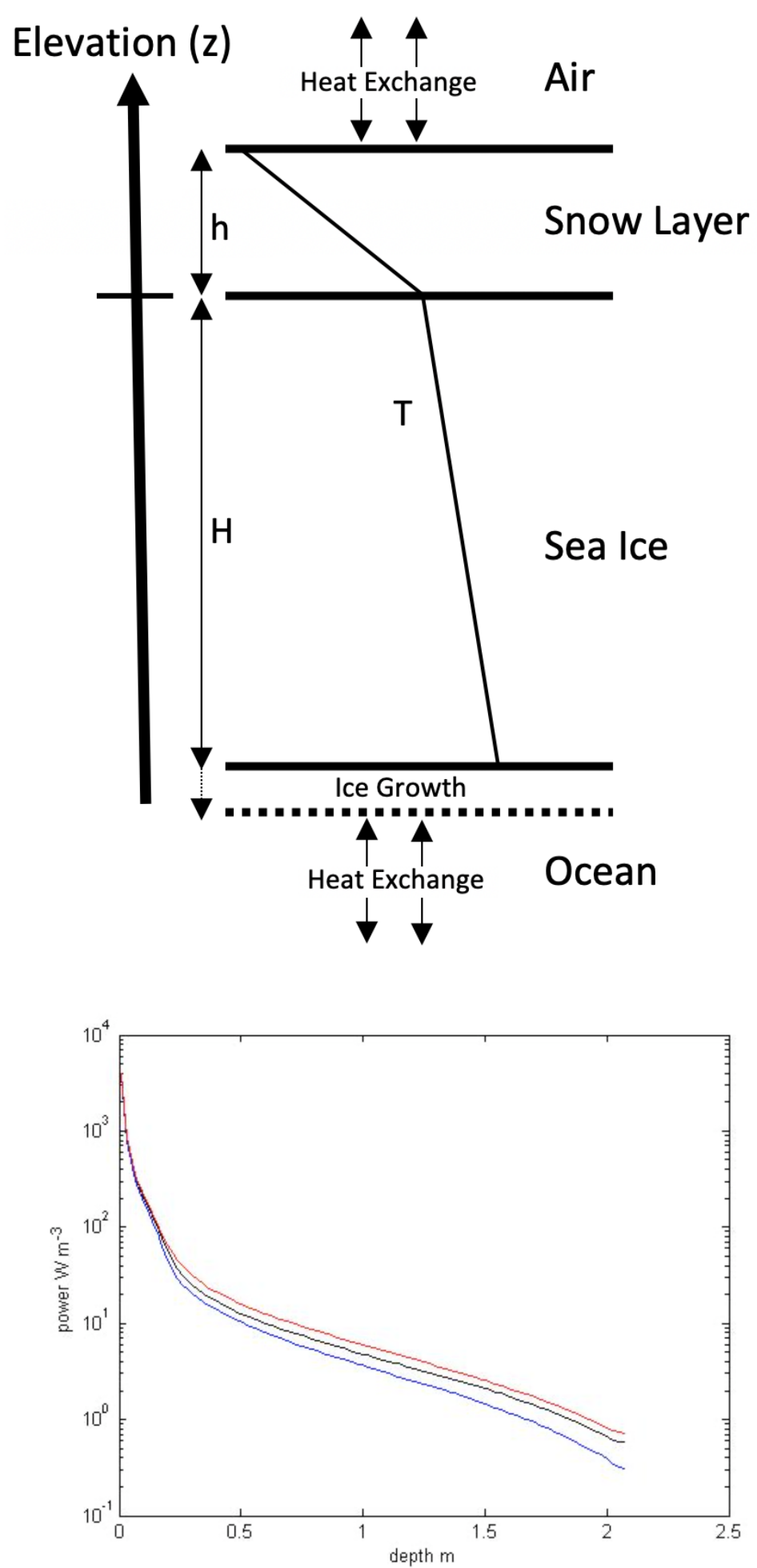

Figure 2.2: Top: Following [63] we consider the dominant heat exchanges between the sea ice, snow, atmosphere and ocean. A steady state temperature profile is shown for constant physical and thermal parameters. Bottom: Reprinted from [69], the solar heating term in sea ice calculated at midday from Monte Carlo photon tracing simulations with depth dependent ice scattering parameters as measured in [26]. Each curve represents a simulation with different scattering parameters as measured in different sea ice locations by $[26]$. 
tude/longitude. The albedo has been parameterized in accordance with CCSM3 as presented, for example, by [59].

If snow is present a similar equation holds for the snow in the region $z \in[0, h]$. Although the heat absorbed due to short wave radiation is mostly only present in spring and summer and may be neglected during the winter (growth phase) months, we include such a term in the above equations for greater applicability.

The boundary conditions these equations must satisfy are derived from balancing the heat transfer at either end of the sea ice/snow. Each of these interactions constitute entire fields of active study, so we present some of the latest parameterizations of these heat transfers below.

- Ocean-ice interface, at $z=H(t)$ the ocean and ice must both be at the freezing temperature

$$
\left.T\right|_{z=H(t)}=T_{\text {freezing }},
$$

assumed to be in perfect thermal contact.

The ice growth rate is determined via a localised steady state approximation at the ice/ocean interface, the heat extracted via the ice dictates the rate at which the liquid freezes modified by an oceanic heat flux

$$
\left.k \frac{\partial T}{\partial z}\right|_{z=H(t)}=\frac{d H(t)}{d t} L \rho_{\text {ice }}+W_{\text {ocean }}
$$

where $L$ is the latent heat of fusion of the ocean and $W_{\text {ocean }}$ is the oceanic heat flux needed to maintain the boundary at the freezing temperature. The oceanic heat flux depends on the conditions of the ocean beneath the ice, and is associated with warm currents or super-cooled water inducing platelet ice growth [95].

- The top temperature of the sea ice (or snow if present) is modelled using an energy balance following the numerical models in the literature cited previously.

$$
\left.k \frac{\partial T}{\partial z}\right|_{z=h(t)}+Q_{d, l w}-\varepsilon \sigma T_{0}^{4}+Q_{s}+Q_{l}=0,
$$

where each term represents: the conductive heat flow from the sea ice/snow, the down-welling long-wave radiation, the up-welling long-wave radiation and the turbulent heat fluxes to and from the atmosphere (sensible and latent). The turbulent heat fluxes are parameterized following [3].

$$
\begin{aligned}
Q_{s} & =\rho_{\mathrm{air}} c_{p, \text { air }} C_{h} v_{\mathrm{ws}}\left(\Theta_{0}-\Theta_{\mathrm{air}}\right), \\
Q_{l} & =\rho_{\mathrm{air}} L_{v, \text { air }} C_{l} v_{\mathrm{ws}}\left(H_{0}-H_{\mathrm{air}}\right),
\end{aligned}
$$


where $\rho_{\text {air }}$ is the density of air, $c_{p}$ is the specific heat capacity of air, $L$ is the latent heat of sublimation, $v_{\text {ws }}$ is the wind speed, $\Theta_{0}$ and $\Theta_{\text {air }}$ are the potential temperatures at the surface of the sea ice/snow and air respectively and $H_{0}$ and $H_{\text {air }}$ are the specific humidity of the surface and the air respectively. $H_{0}$ is interpreted as the saturation humidity at the surface temperature. $C_{l}$ and $C_{h}$ are the bulk coefficients of turbulent latent and sensible heat transfer respectively, and depend on the stability of the atmosphere, roughness lengths of temperature, humidity and windspeed. Assuming that the roughness lengths of humidity and air temperature are equal reduces $C_{h}=C_{l}[3,4]$. We compute $C_{h}$ using the stability functions proposed in [25] based on the bulk Richardson number $R i_{b}$ and the neutral-limit turbulent Prandtl number, $P r_{0}$, a parameterization based on the SHEBA dataset. Other parameterizations, such as the ones discussed in [61], may be used alternatively.

Unlike other models in the literature [52,64], we do not include melt pond formation, but rather raise a warning for times where simulated temperatures were higher than the melting temperatures. This is due to minimal melt occurring during the winter months for which this model is valid.

- If snow is present, at the ice/snow interface the temperature is set to be continuous and the heat flux from either side must balance

$$
\left.T_{\text {ice }}\right|_{z=0}=\left.T_{\text {snow }}\right|_{z=0},\left.\quad k_{\text {ice }} \frac{\partial T_{\text {ice }}}{\partial z}\right|_{z=0}=\left.k_{\text {snow }} \frac{\partial T_{\text {snow }}}{\partial z}\right|_{z=0} .
$$

\subsection{Discretization and Freezing the Boundary}

The domain over which the heat equation is to be solved is changing as the ice grows or melts. A transformation of the depth variable allows an equivalent problem to be solved over a fixed domain [49] (a frozen boundary, a fitting name for our application). This adds ease in numerical computations and avoids errors associated with interpolation when the boundary moves which could introduce unknown errors. Both the ice/ocean boundary and the snow/air boundary coordinates are frozen by defining the new depth variables $\xi=\frac{z}{H(t)}$ for ice and $\chi=\frac{z}{h(t)}$ for snow, so that the snow / air boundary is fixed at $\chi=1$, the ice/ocean boundary is fixed at $\xi=1$, and both coordinates have their origin at the ice/snow boundary. For all times, the temperature profiles are now represented on the depth ranges $\xi, \chi \in[0,1]$. 
The chain rule for changing the variables are as follows, in the ice

$$
\begin{aligned}
\left.\frac{\partial}{\partial t}\right|_{z} & =\frac{\partial}{\partial t}+\frac{\partial \xi}{\partial t} \frac{\partial}{\partial \xi}=\frac{\partial}{\partial t}-\xi \frac{\dot{H}(t)}{H(t)} \frac{\partial}{\partial \xi} \\
\frac{\partial}{\partial z} & =\frac{\partial \xi}{\partial z} \frac{\partial}{\partial \xi}=\frac{1}{H(t)} \frac{\partial}{\partial \xi}
\end{aligned}
$$

and similarly in the snow.

With reference to the equations in section 2.2, the transformed heat equation reads

$$
\frac{\partial T}{\partial t}=\frac{1}{\rho c H(t)^{2}} \frac{\partial}{\partial \xi}\left(k \frac{\partial T}{\partial \xi}\right)+\xi \frac{\dot{H}(t)}{H(t)} \frac{\partial T}{\partial \xi}+\frac{1}{\rho c} P(H(t) \xi, t), \quad \xi \in[0,1],
$$

for ice, and similarly for snow.

We know apply the method of lines [87] by discretizing over the depth, turning the partial differential equation into many coupled ordinary differential equations. A mesh of constant spacing $\Delta \xi, \Delta \chi$ is used on the reparameterized depth variables. Typical values for these are between 0.005 and 0.1 . This prepares the approximate solution to be easily attained using numerical computations. The spatially varying parameters are labeled as follows at these depths

$$
T_{i}=T\left(\xi_{i}, t\right), \rho_{i}=\rho\left(\xi_{i}\right), c_{i}=c\left(\xi_{i}\right), k_{i}=k\left(\xi_{i}\right), \xi_{i}=i \times \Delta \xi, i=0,1, \ldots, n
$$

and the spatial derivatives are approximated using central differences

$$
\frac{\partial}{\partial \xi}\left(k \frac{\partial T}{\partial \xi}\right) \approx \frac{k_{i+1 / 2}\left(\frac{T_{i+1}-T_{i}}{\Delta \xi}\right)-k_{i-1 / 2}\left(\frac{T_{i}-T_{i-1}}{\Delta \xi}\right)}{\Delta \xi},
$$

where we note that the thermal conductivity is to be evaluated 'in between' the discretized points. This is done by interpolating on the temperature profile

$$
k_{i \pm 1 / 2}=k\left(\frac{\xi_{i}+\xi_{i \pm 1}}{2}\right) \text {. }
$$

The coupled ordinary differential equations representing the evolution of the temperature profile are approximated by

$$
\begin{aligned}
\frac{d T_{i}}{d t}= & \frac{1}{\rho_{i} c_{i} H^{2}} \frac{k_{i+\frac{1}{2}}\left(T_{i+1}-T_{i}\right)+k_{i-\frac{1}{2}}\left(T_{i-1}-T_{i}\right)}{(\Delta \xi)^{2}} \\
& +\xi \frac{\dot{H}}{H}\left(\frac{T_{i+1}-T_{i-1}}{2 \Delta \xi}\right)+\frac{1}{\rho_{i} c_{i}} P\left(H \xi_{i}, t\right), i=1 \ldots n-1 .
\end{aligned}
$$

Similarly, the temperatures in the snow, $T_{j}^{s}$, must satisfy

$$
\begin{aligned}
\frac{d T_{j}^{s}}{d t}= & \frac{1}{\rho_{j}^{s} c_{j}^{s} h^{2}} \frac{\left(k_{j+1}^{s}+k_{j}^{s}\right)\left(T_{j+1}^{s}-T_{j}^{s}\right)+\left(k_{j-1}^{s}+k_{j}^{s}\right)\left(T_{j-1}^{s}-T_{j}^{s}\right)}{2(\Delta \chi)^{2}} \\
& +\chi \frac{\dot{h}}{h}\left(\frac{T_{j+1}^{s}-T_{j-1}^{s}}{2 \Delta \chi}\right)+\frac{1}{\rho_{j} c_{j}} P\left(H \chi_{j}, t\right), j=1 \ldots m-1 .
\end{aligned}
$$


These equations, together with the discretized version of equation 2.4 ,

$$
\frac{d H}{d t}=\left(\frac{k_{n-1 / 2}}{L \rho_{n-1 / 2}}\right)\left(\frac{T_{n}-T_{n-1}}{H \Delta \xi}\right)-\frac{W_{\text {ocean }}}{L \rho_{n-1 / 2}},
$$

are to be solved simultaneously for the unknown temperatures $T_{i}(t)$ and $T_{j}^{s}(t)$, $i=1 \ldots n-1, j=1 \ldots m-1$, and for the unknown $H(t)$. The subscripts on $\rho$ are to be interpreted the same as those on $k$.

The user provides $h(t)$. If no snow layer is desired, $h$ may be set to a relatively small number so that the snow layer has negligible effect. This allows snow to be added at a later time after being removed, and through numerical testing was found to not affect the solution appreciably. Solving requires that the boundary values $T_{0}, T_{n}, T_{0}^{s}$, and $T_{m}^{s}$ are known. These are provided by the discretized version of the boundary conditions, equations $2.3,2.5$ and 2.6 .

- At the ice/ocean interface, $T_{n}=T_{\text {freezing }}$.

- The upper temperature is solved for numerically to satisfy the following

$$
k_{1 / 2} \frac{T_{0}-T_{1}}{H \Delta \xi}+Q_{d, l w}-\varepsilon \sigma T_{0}^{4}+Q_{s}+Q_{l}=0
$$

with the down-welling longwave radiation set by the user as a time-series, and the turbulent heat fluxes calculated using the previously stated parameterizations based on user provided atmospheric conditions.

- The ice/snow interface boundary condition at origin is given by $T_{0}=T_{0}^{s}$, and

$$
k_{0}\left(\frac{T_{1}-T_{0}}{H \Delta \xi}\right)=k_{0}^{s}\left(\frac{T_{1}^{s}-T_{0}^{s}}{h \Delta \chi}\right) .
$$

Solving for $T_{0}$ gives:

$$
T_{0}=\frac{H k_{0}^{s} T_{1}^{s} \Delta \xi-h k_{0} T_{1} \Delta \chi}{H k_{0}^{s} \Delta \xi-h k_{0} \Delta \chi} .
$$

Equations 2.9, 2.10, and 2.11 are solved simultaneously together with the boundary conditions, using Matlab's stiff ordinary differential equation solver ode $15 \mathrm{~s}$. The system at hand is stiff, most easily seen through the varying time scales present, parts of the solution can vary quickly, such as during rapidly changing atmospheric conditions, while other parts vary more slowly [88]. More details on the numerical simulation may be found in the code with documentation on GitHub [8]. 


\subsection{Validating the Model}

We compare simulated temperatures to field measurements of thermistor strings from both the Arctic and Antarctic available at online repositories. Thermistor string data from [71], labeled AR, and measurements undertaken in McMurdo Sound, Antarctica taken from https: / / seaice.alaska.edu/gi/data/mcmurdo/ [75, 103], labeled AN, are used for comparison. Here we demonstrate such comparisons with the measurements undertaken in McMurdo Sound, Antarctica, during 1997. Temperature profiles of the sea ice were obtained by placing thermistors on a $2 \mathrm{~m}$ rod at intervals of $10 \mathrm{~cm}$ and freezing it into relatively fresh $90 \mathrm{~cm}$ thick sea ice in early June. Thermistor temperatures were recorded every half-hour to a data-logger until removal early in December. Further details such as the instrumentation and location can be found in [103]. We estimate the oceanic heat flux from the measurements and provide it to the simulation, as is detailed in the following analysis.

\subsubsection{Ice Thickness Estimation}

At each time, we use the temperatures measured with the thermistor array following the methods used by [24] as illustrated in Figure 2.3, to estimate ice thickness. The thermistors are divided into two groups, labeled ice and ocean. Initially all thermistors are in the ocean group. A linear line is fitted to the four thermistors closest to the ocean and the intersection of this line and the freezing temperature, being the mean of the ocean thermistors, is found. If the intersection is between the deepest ice thermistor and the highest ocean thermistor, the intersection is saved as the sea ice boundary. If not, the lowest ice thermistor is transfered to the ocean thermistor group and the process repeated. We further check the mean of the four ice thermistors closest to the ocean is lower than the mean of the ocean thermistors to increase reliability. This assumes a 'clean boundary' between the ice and the ocean, ignoring the effects of a possible mushy layer due to the presence of brine channels or a sub-ice platelet layer [95, 111, 112].

By smoothing the estimates of ice thickness $H$ using a 7 day running average and differentiating with respect to time, an estimate for the growth rate $\dot{H}$ is also obtained directly from thermistor data for comparison purposes. Smoothing is necessary as the data is noisy, giving high variability in growth rates otherwise. 


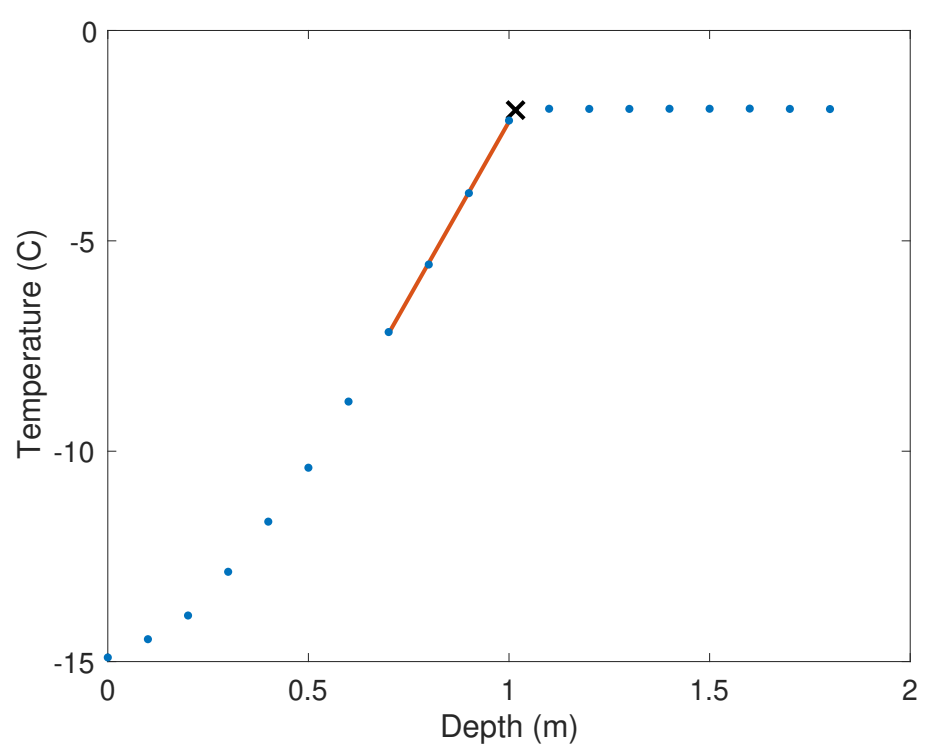

Figure 2.3: A sketch illustrating how the data is used to infer the location of the ice-ocean interface following [24]. A local freezing temperature is calculated directly from the data. The intersection between this and a line fitted to the last four points within the ice gives the estimated interface location (marked by the black $x$ ). The gradient of the temperature profile at the interface is estimated as the slope of the fitted red line.

\subsubsection{Estimating Oceanic Heat Flux}

The data-based temperature gradients are used together with data-based estimates of $H$ and $\dot{H}$, to derive the elusive oceanic heat flux term, following [24,32], by balancing the heat fluxes in a reference layer $15 \mathrm{~cm}$ above the base of the sea ice.

$$
W_{\text {ocean }}=-\left.k \frac{\partial T}{\partial z}\right|_{z=z_{r}}+\left.\rho c \Delta H \dot{T}\right|_{z=z_{r}}-\dot{H} L \rho .
$$

For AN1997, a significant transition to a negative oceanic heat flux may be seen in Figure 2.4 when the ice is about 1.4 meters thick, approximately at day 40. This coincides with the first appearance of platelet ice in ice cores [103], as seen in Figure 2.5.

\subsubsection{Numerical Simulation of Ice Temperatures}

The simulator was run, using thermistor measurements at time zero to provide the initial temperature profile for starting the simulation. Since the uppermost thermistors are inside snow, the (modeled) upper boundary condition is set via 


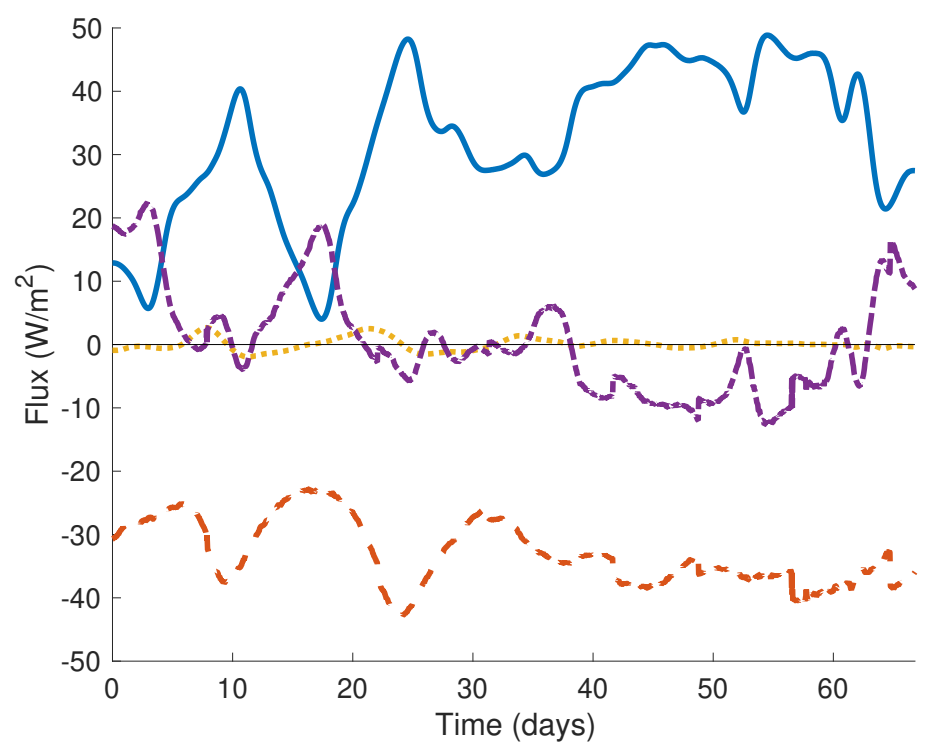

Figure 2.4: Heat flux balance near the bottom of the sea ice is used to calculate the oceanic heat flux for the AN1997 thermistor. The contributions are as follows. Solid blue line: latent heat of freezing. Dashed red line: conductive heat into the ice. Dotted yellow line: internal heating of the reference layer as its temperature changes. Dashed purple line: The oceanic heat flux calculated from equation 2.14

the first thermistor in the sea ice. A snow layer is then not needed and is not simulated in this section, which tests the accuracy of our numerical simulator for heat transport solely within the sea ice. The surface temperature is forced onto the upper ice thermistor.

We test the sensitivity of the simulation to the salinity profile by comparing simulations with the same forcing parameters. Presented is the difference between the simulated measurements using the forcing parameters of AN1997, with a salinity of $10 \mathrm{ppt}$ and $2 \mathrm{ppt}$. A maximal difference in the temperature field of $1^{\circ} \mathrm{C}$ is observed, and the subsequent changes to the ice-ocean interface evolution result in about a $6 \mathrm{~cm}$ difference after 100 days of simulation, as seen in Figure 2.6 .

The differences between simulated and measured temperatures are plotted in Figure 2.7, with the ice-ocean interfaces overlaid. The comparisons shown indicate very good agreement between the simulated and measured temperatures within the sea ice, to approximately a $1^{\circ} \mathrm{C}$ accuracy in regions of sea ice growth. For times of sea ice melt we observe large discrepancies, seen as large blue sections in Figure 2.7. This is due to the lack of convective heat transport in the simulation, associated with the ocean and/or melt ponds flushing the sea ice. 


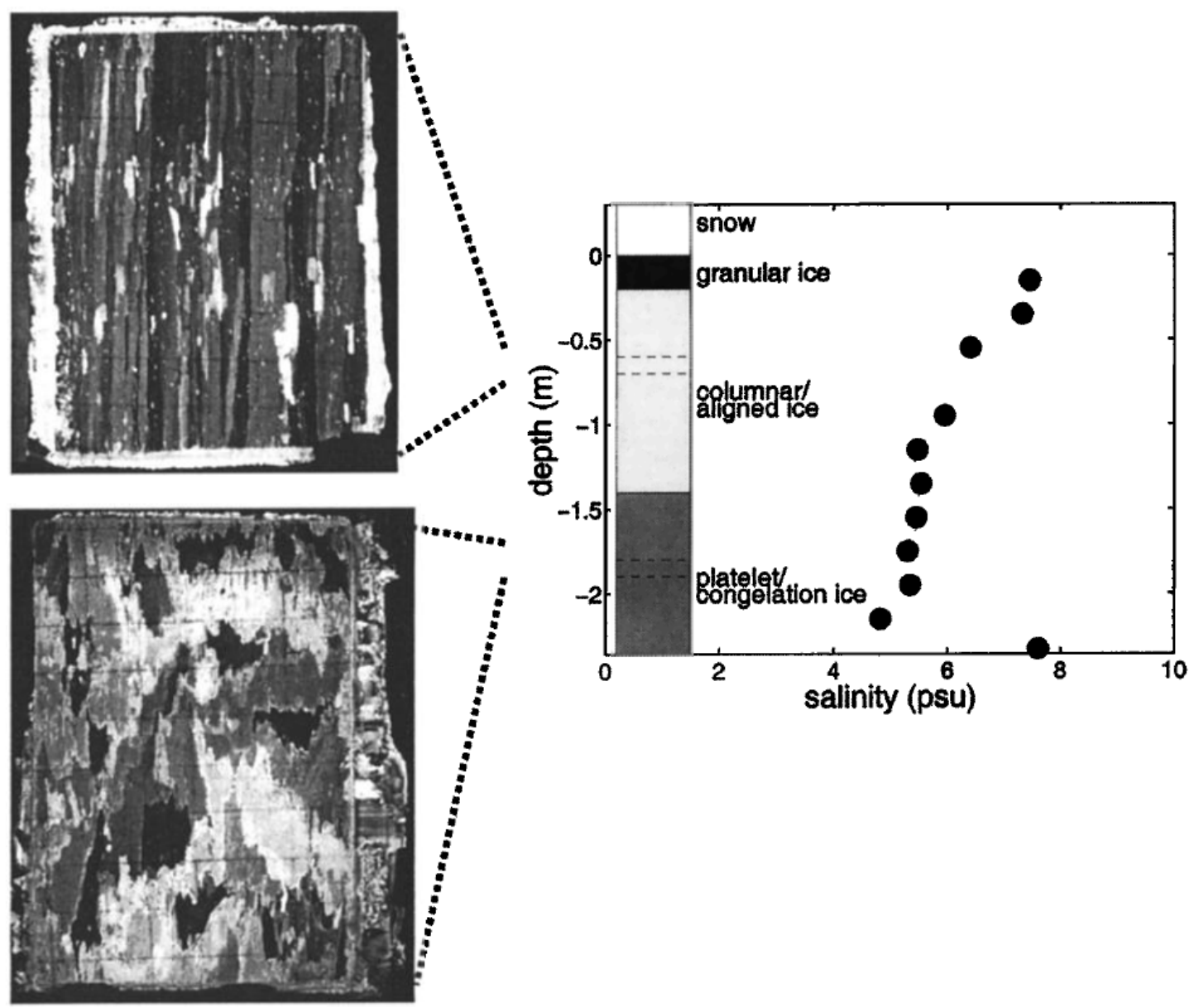

Figure 2.5: Reprinted from [103]. Ice core data taken near the temperature array AN1997 in November 1997. The crystal orientations, viewed under crossed polarisers, change from aligned to random, giving insight to the presence or absence of platelet ice. 

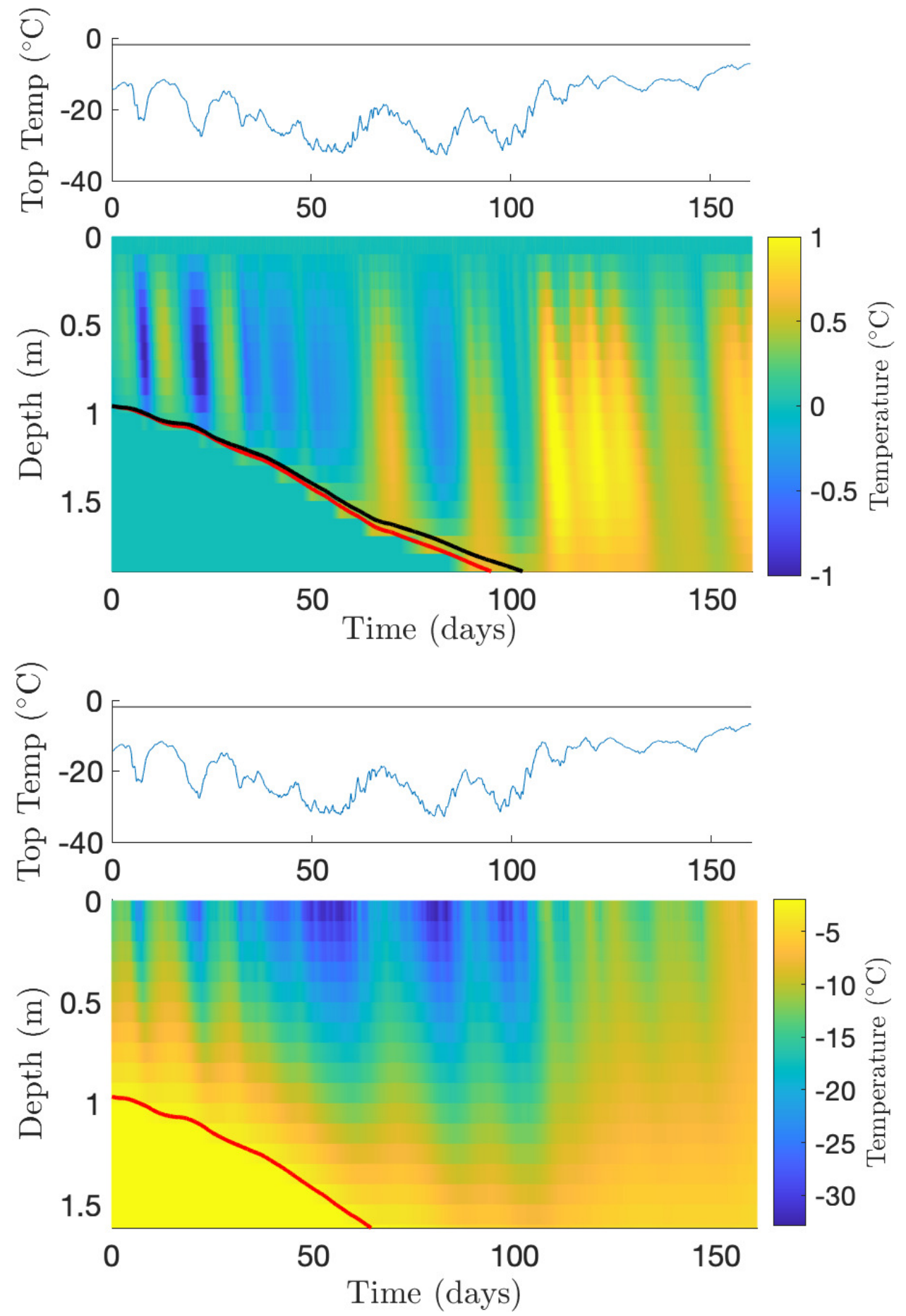

Figure 2.6: Top: Forcing data from thermistor AN1997 is used to compare the sensitivity of the model to the input salinity. The colours represent the difference between the simulated temperatures $T_{2 \mathrm{ppt}}-T_{10 \mathrm{ppt}}$ where the subscript denotes the constant salinity value used in the simulation. Overlaid is the calculated ice/ocean interface for the $2 \mathrm{ppt}$ salinity (red) and 10 ppt salinity (black). Bottom: The calculated temperature profile for the simulation with a salinity of $2 \mathrm{ppt}$. 
These events usually occur when the sea ice is warming above the freezing temperature. The large discrepancy around days 80-90 of AN1999 occur at times of a noted instrumentation failure inducing a lack of data at these times, with linear interpolation being used to fill the gap in the data.
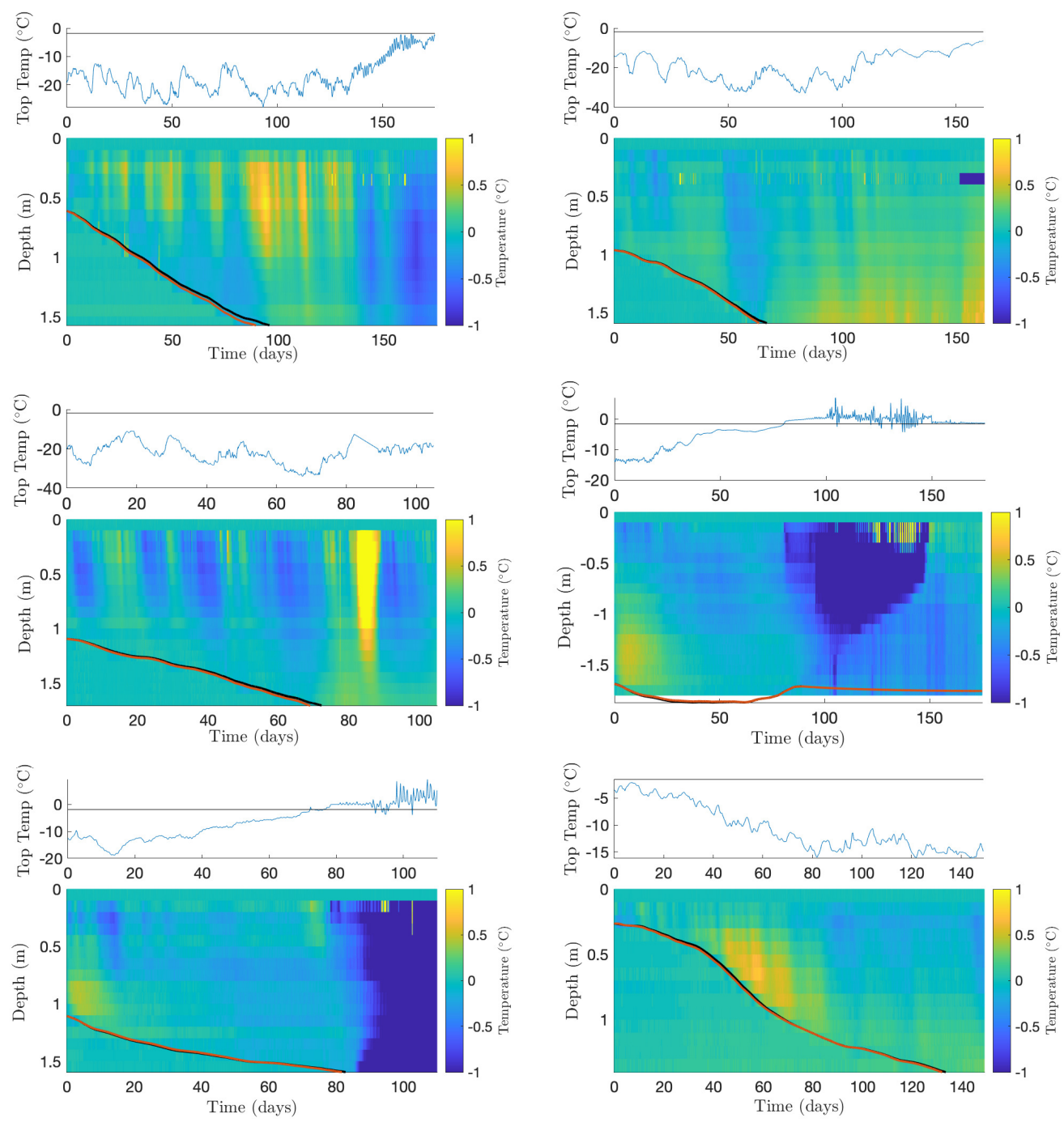

Figure 2.7: Comparison between the simulated and thermistor array measurements. The surface temperatures is plotted as a blue line above each thermistor array comparison, and the colour represents the discrepancy $T_{\text {simulated }}-T_{\text {data }}$ in the various thermistor arrays. Overlaid are the measured sea ice-ocean interface (black line) and the simulated ocean interface (red line). From left to right, then top to bottom, the thermistor arrays are: (1) AN1996, (2) AN1997, (3) AN1999, (4) AR2014C, (5) AR2015A, (6) AR2015G

The striped colour patterns visible in Figure 2.7 during conductive dominated heat transport regions represent travelling waves of small discrepancies in temperature, possibly due to uncertainties in the salinity values affecting the ice's 
thermal properties. 


\subsection{Further Application: Snow Cover}

Snow may be added to the model by the user and can vary in thickness with time as desired. New snow is automatically added at the current air temperature, and snow is removed at the temperatures that currently apply inside the removed snow.

We compare our model simulated results to sea ice growth rates measured via banding analysis performed on an ice core from 1999 in McMurdo Sound, Antarctica, and a previously used steady state model as described in [104]. The banding analysis uses dark bands sometimes seen in young sea ice to infer sea ice growth rate as a function of thickness and can be used to measure growth rates for thin sea ice when a thermistor string cannot safely be deployed. An ice thickness of $110 \pm 5 \mathrm{~cm}$ was measured using data from a nearby thermistor string [94,104] on 21 June. Congelation ice formation started at $15 \mathrm{~cm}$ thickness. The models were tuned to match the sudden rise in air temperatures causing a drop in the growth rates near a thickness of $60 \mathrm{~cm}$ and to match the final ice thickness. The model presented in [104] was tuned with a freeze in time (15 cm thickness of ice) of the early hours of 14 May. Our simulation with no snow had a freeze in time of the early hours of 15 May and with a $15 \mathrm{~mm}$ snow cover a freeze in time of midday on 11 May. The final thicknesses on 21 June of the simulations were $110 \mathrm{~cm}$ with no snow and $105 \mathrm{~cm}$ with $15 \mathrm{~mm}$ of snow. Both of these are within the measured value range.

With no snow cover the dynamic simulation overestimates the banding-inferred growth rates, and the steady-state model presented in [104] (which also made no allowance for a snow cover) fits the early growth rates more closely than our transient model. This may be due to the different freeze in dates selected to tune each model. When the same freeze in date is selected the transient rates are a closer match to the steady state rates early in the season, but they deviate from the steady state rates later in the season. Furthermore, the latent heat of sea ice used in the steady state model is higher than what we would expect [115] at the typical temperature and salinity of near-freezing sea water, inhibiting the overall modeled growth rates of the steady-state model used.

A constant $15 \mathrm{~mm}$ thick snow cover in the dynamic simulations produces better agreement with banding-inferred growth rates than the no snow models, as may be seen in Figure 2.8. The match is surprisingly good over both early and late times, given the simplicity of the assumption that the snow cover is constant in time. The expected effect of the presence of a snow cover is clearly seen, reduc- 
ing ice growth rates compared to when there is no snow cover, especially earlier in the season.
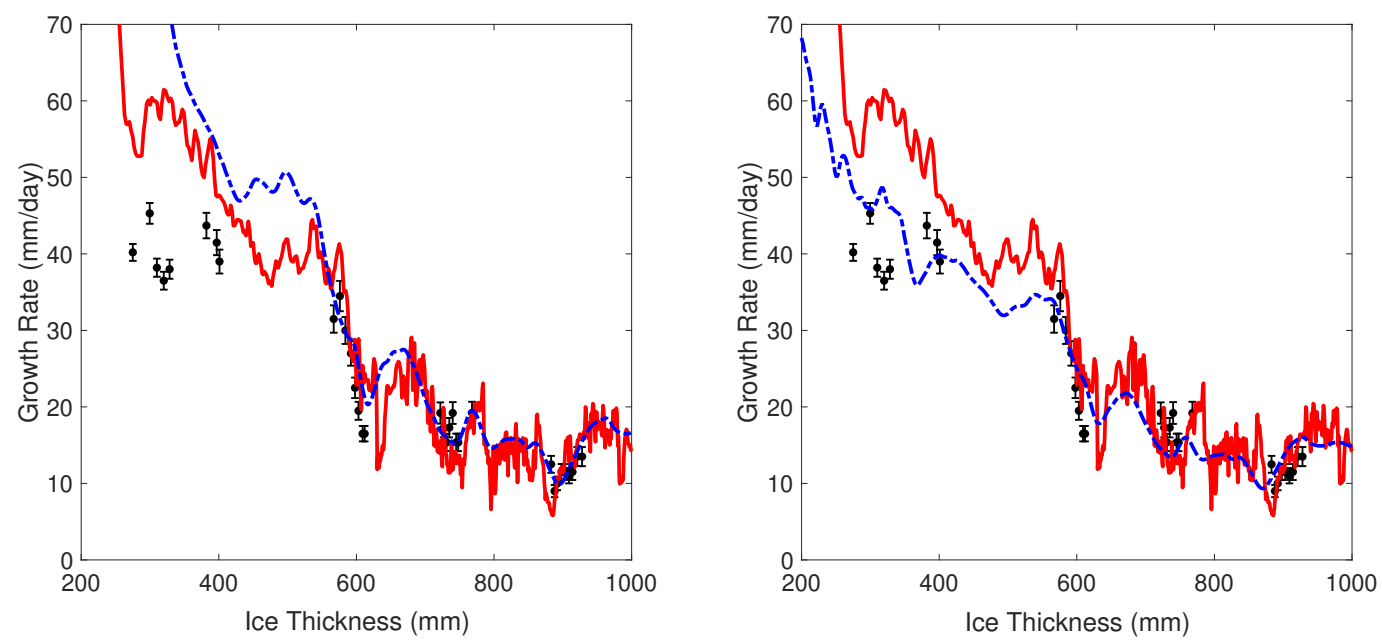

Figure 2.8: A comparison of ice growth rates computed using a steady state model as described in [104] (solid red curve) with growth rates computed using the new fully transient model presented in this chapter (dashed blue curve). Data values are computed from a banding analysis of ice cores (black symbols) [104]. Air temperatures used are those given in [104] Left: No snow layer is present in the transient simulations (dashed blue curve). Right: A snow layer of constant $15 \mathrm{~mm}$ thickness has been included in the transient simulations (dashed blue curve).

The ice core with banding analysis performed to get these measurements was taken about $1 \mathrm{~km}$ offshore near Arrival Heights in Antarctica in 1999 at a site $150 \mathrm{~m}$ away from a thermistor string at a latitude/longitude of $77^{\circ} 50.197^{\prime} \mathrm{S}, 166^{\circ} 36.764^{\prime} \mathrm{E}$. The thermistor string used has been described in [95] and analysed in [103]. The air temperatures measured at Scott Base [70] were used as a proxy for the air temperatures above the ice for the simulations. Some snow cover was observed to be present at the site when ice cores were taken.

\subsection{Summary}

In this chapter we presented a numerical tool for calculating temperature transients in sea ice and snow based on models developed in the literature. We use parameterizations of the thermal and physical properties of sea ice and snow in the literature. The model is implemented in MATLAB and is numerically solved using the built in stiff differential equation solver ode15s. 
The mechanics of the model include using a coordinate transformation to solve an equivalent heat conduction problem on a fixed boundary (with "frozen" coordinates). The method of lines is used to discretize over a spacial mesh and convert the partial differential equation to a set of coupled ordinary differential equations. The code is made available on GitHub [8] and other online repositories.

The model is compared to thermistor string measurements with inclusion of estimated ocean flux from the data. We see excellent transient treatment, with an agreement of $1{ }^{\circ} \mathrm{C}$ within the entire thermistor array during times of conductive heat transport. Discrepancies between the model and the data highlight times of non-conductive heat transport or occur at times of measurement equipment failures. The ice depth is recreated well throughout the growth stage.

Further comparison to sea ice growth rates from McMurdo Sound, Antarctica in 1999 obtained from ice core banding patterns indicate the need of a previously speculated $15 \mathrm{~mm}$ layer of snow. 


\section{Chapter 3}

\section{Oscillatory Temperatures in Sea Ice}

Solar radiation heats up the sea ice over the summer months and is a contributor to sea ice melt [1,74]. Understanding the solar radiation's interaction with the sea ice is vital to better understand the late-season thermodynamics and how sea ice mediates the solar radiation absorption of the ocean. Although a significant portion of the incident solar radiation is reflected at the surface of the sea ice [105], radiation can penetrate deep into the snow and ice and when absorbed adds a distributed heat source. Parameterizations of this interaction (such as the amount of radiation entering the sea ice, the absorption over depth, radiation heating up the ocean below etc.) are all important parameters to understand due to their effect on solar heat absorption by the sea ice and the ocean. These also dictate the thermal wave behaviour present in the sea ice induced by the diurnal oscillation of the solar radiation.

In this chapter we follow the work presented in [66] to derive the leading order behaviour of the thermal waves due to the absorption of solar radiation within the sea ice. We start by introducing Monte Carlo photon tracing simulations run by [69], giving estimates for the distributed source term $P(z)$. Data analysis is presented to motivate the study of the thermal waves generated by such a distributed source and an oscillatory boundary condition. Analysis of the amplitude and phase of thermal waves present in thermistor data from 1996, 1999 and 2003 is presented. We then derive a differential equation for the amplitude and phase of the thermal waves generated by the distributed heating term and oscillatory boundary conditions. 


\subsection{Background}

During the summer months where the sun rises above the horizon in Antarctica the azimuth (angle above the horizon) of the sun has a 24-hour periodicity with a slow drift throughout the year. The amount of solar radiation that enters the sea ice depends on the atmospheric and surface conditions, as well as the conditions of the ice, such as its thickness. Clouds and other particles in the air scatter and absorb sun light and can cause both a decrease or increase (atmospheric lensing) in the solar radiation entering the sea ice. The presence of snow or meltponds significantly changes the albedo, being the ratio of reflected to incident sunlight.

As the sunlight enters the sea ice it is scattered and absorbed. For a homogeneous material, the absorbed power per unit volume follows Beer's law [11], an exponential decay. As was discussed in the introduction, sea ice is a complex, inhomogeneous material. The scattering and absorption of the light is very complex and can vary significantly for various ice locations, growth types etc. Most significantly, the lack of a uniform crystal orientation in the upper sections of the ice slab, where frazil ice growth occurred, causes significant scattering and absorption in the upper $\approx 20 \mathrm{~cm}$ of sea ice.

Monte Carlo photon tracing simulations have been used to investigate the solar radiation absorption of sea ice [55, 57, 58, 69]. In [69] such simulations were setup based on scattering and absorption parameters of sea ice that are depth and wavelength dependent, as measured in [26,102]. This gives the distributed source term $P(z)$ that we will use in our analysis. As seen in Figure 3.1 the distributed heating source can be well approximated by an exponential decay with a fixed decay coefficient in two regions, one very near the surface to a depth of about $20 \mathrm{~cm}$ and the other from $20 \mathrm{~cm}$ and deeper. As has been studied in [19, 69], the inclusion of algae, air bubbles and other impurities can increase absorption at depths, effectively decreasing the decay rate of the distributed source. In [66, 103] it was noted that the absorption of sunlight by the therimstors is negligible and inclusion of impurities in the ice is necessary to explain the decay of the thermal waves measured.

This distributed heating term has been linked to daily oscillations measured in the sea ice thermsitor arrays. Such examples are shown in Figures 1.1 and 3.2 . These measured thermal waves with a periodicity of 24 hours are also due to a second prominent driving force - diurnal oscillations of the air temperatures. Air temperature measurements from Scott Base in 1997 retrieved from [70] shown in Figure 3.3 indicate typical large weather system variations overlaid with smaller 


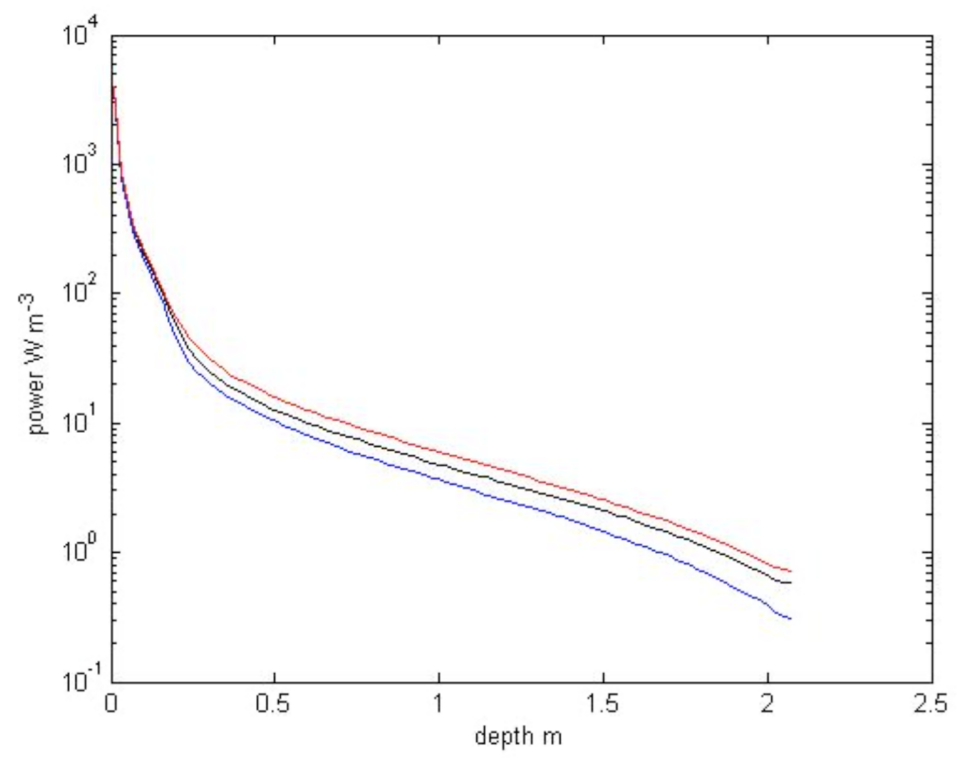

Figure 3.1: Reprinted from [69], the solar heating term in sea ice calculated from Monte Carlo photon tracing simulations with depth dependent ice scattering parameters as measured in [26]. Each curve represents a simulation with different scattering parameters as measured in different sea ice locations by [26].

daily variation. Air temperature fluctuations and incoming solar radiation measurements from Scott Base in 1999, also retrieved from [70], show a slight lag between of the daily temperature cycle as compared with the solar radiation, seen in Figure 3.4. Air temperatures were processed with a high pass filter to extract the daily (and sub-daily) temperature fluctuations.

We present measured daily thermal oscillations from the thermistor strings in the sea ice, following the work of [66] and using the thermistor strings referenced in Chapter 2. We extract the thermal wave amplitude and phase by applying a highpass frequency filter to the timeseries of each thermistor. Oscillations of frequency slower than 1 wave/day are filtered out. Care was taken in this process by using a zero phase filter, ensuring that the phase of the thermal waves is not compromised. An example of the result of such analysis is displayed in Figure 3.5. Similar analysis consisting of a fitted background temperature profile from daily averages being subtracted results in very similar thermal wave profiles.

We see very clearly that two regions are present with different thermal wave behaviour. The first is a quick decay of travelling thermal waves (with a varying phase in relation to depth) up to a thickness of $\approx 50 \mathrm{~cm}$. The second region consists of a slower decay of the thermal waves at a fixed phase. The travelling thermal waves have been attributed to the quick decay in the absorbed power at shal- 


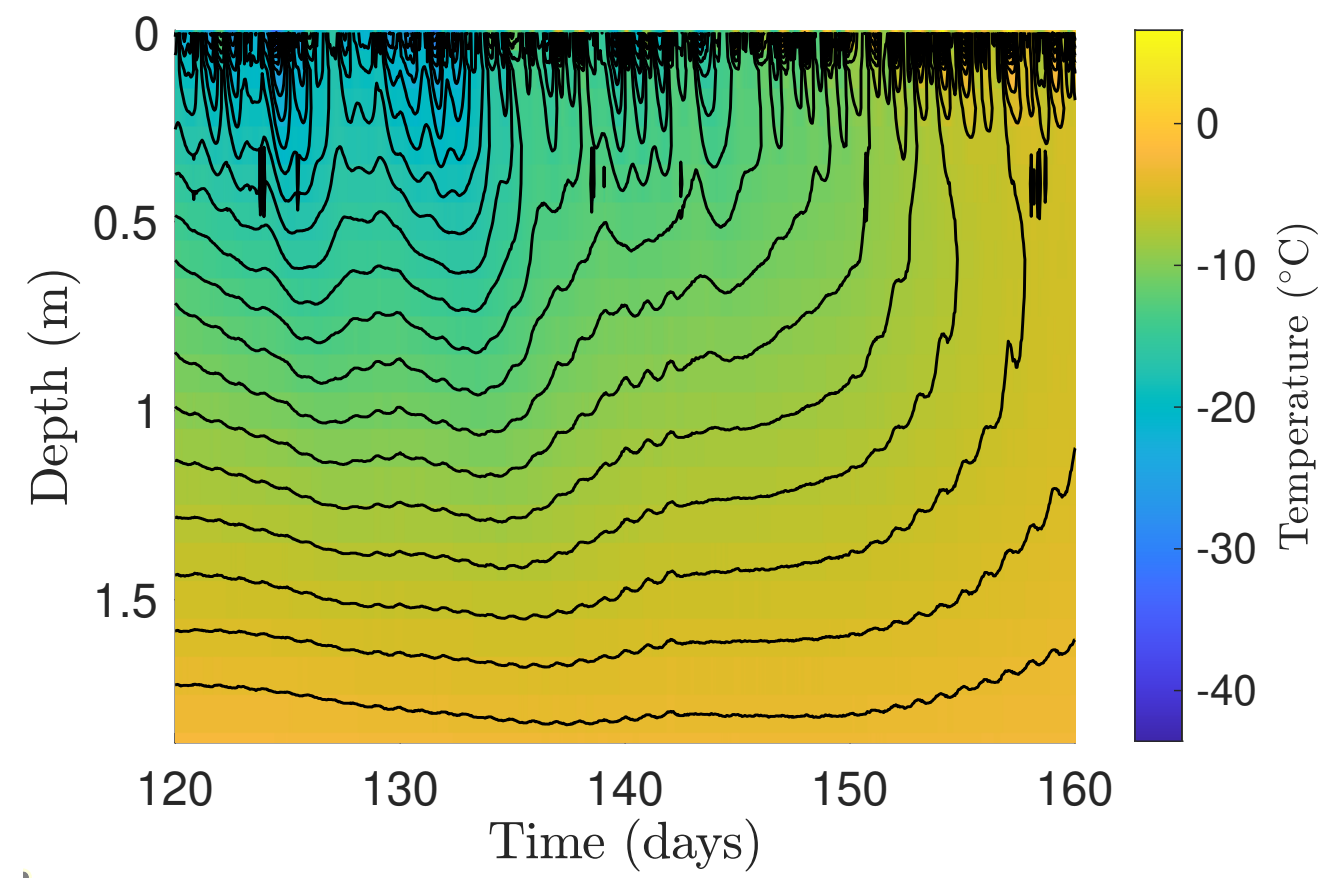

Figure 3.2: The AN1996 thermistor data overlaid with contours. The daily cycle appears both near and far from the top surface approximately from day 120, with small daily oscillations appearing over the 'background' temperature profile at depths deeper than $0.5 \mathrm{~m}$.
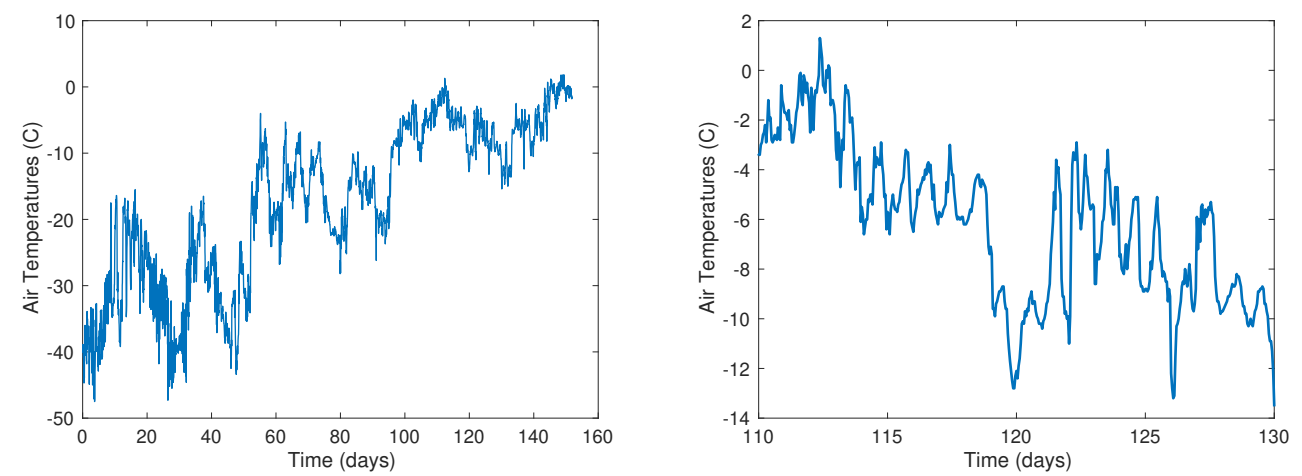

Figure 3.3: Air temperatures as measured in Scott Base, Antarctica from 01 August-31 December 1997 (left) and a close up highlighting the daily oscillations measured (right). 


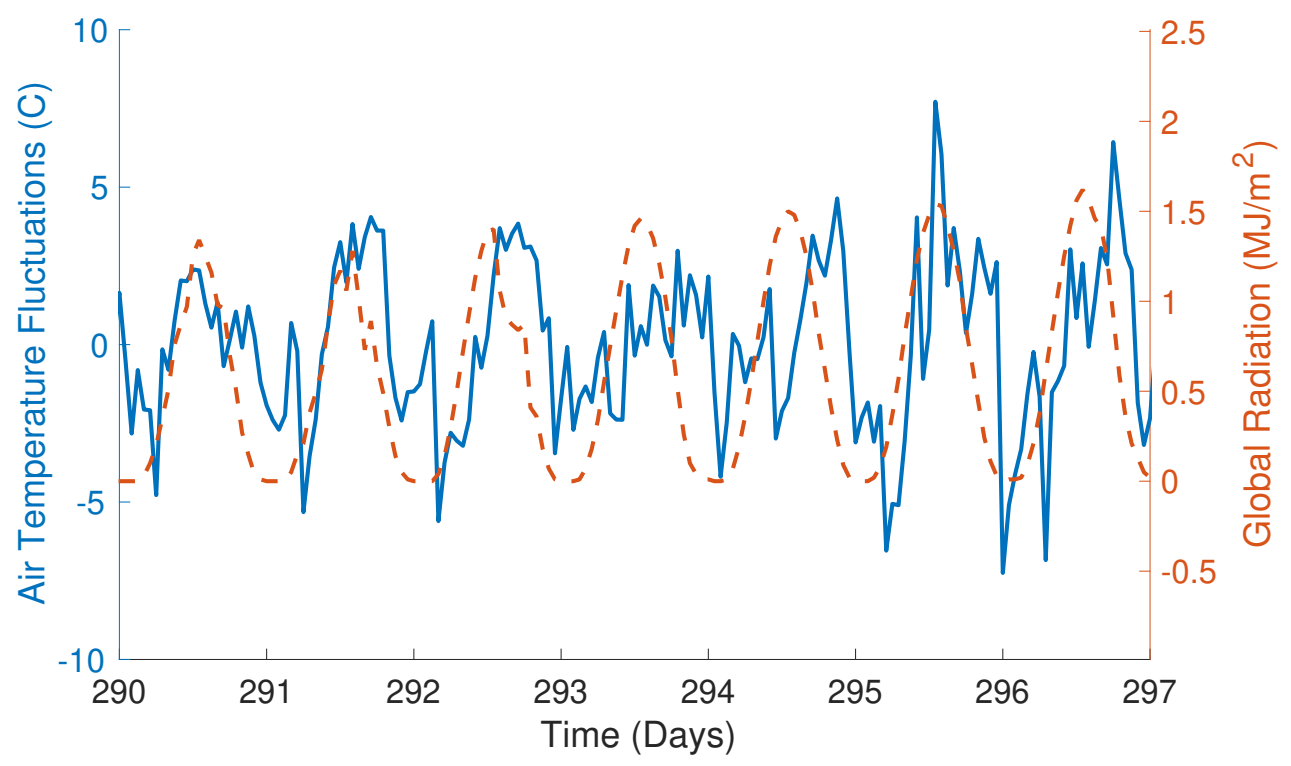

Figure 3.4: Air temperature fluctuations (solid blue) and global (direct plus diffuse) radiation falling on the surface of Antarctica (dashed red) as measured at Scott Base, Antarctica in 1999.

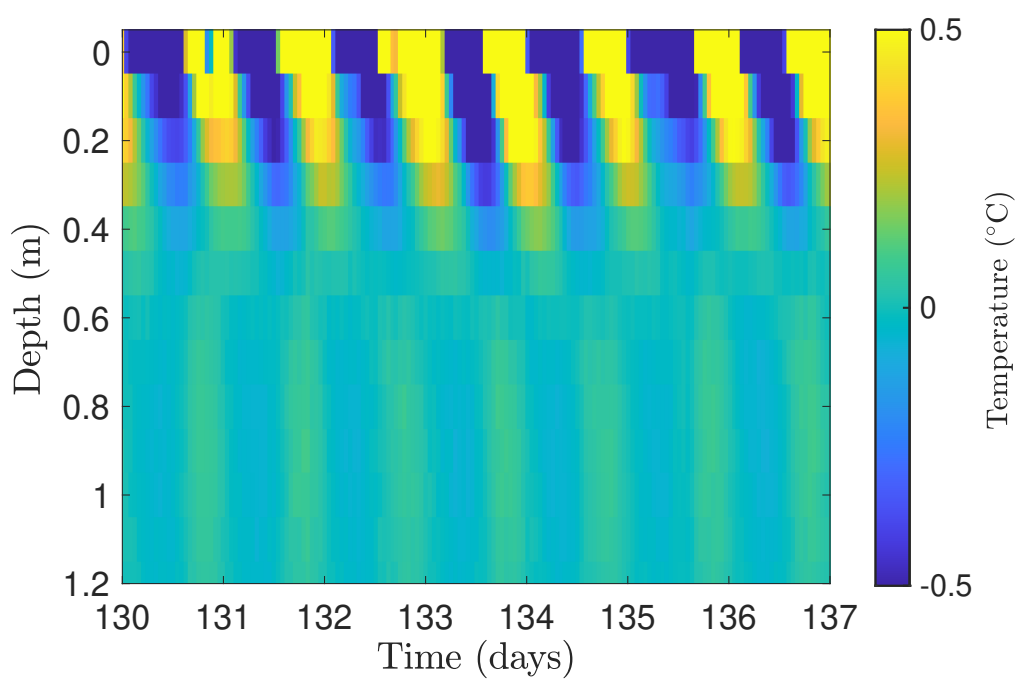

Figure 3.5: The measured daily fluctuations in the AN1996 thermistor array. The colour map shows the temperature fluctuation from the average background temperature profile. We clearly see the travelling thermal waves near the surface of the sea ice and the stationary thermal waves at deeper depths (the bottom $0.8 \mathrm{~m}$ of data not shown) with a relatively constant amplitude. It is interesting to note that the waves destructively interfere near a depth of $\approx 0.5 \mathrm{~m}$. The colour map has been saturated to ensure the stationary waves are visible. 
lower depths inducing a heat imbalance that decays into the ice over time [66]. We add that an oscillatory boundary condition, such as oscillating air temperatures, also induces a travelling thermal wave and the observed travelling thermal wave will be a combination of the atmosphere induced and solar radiation induced travelling waves. The deeper stationary waves are due to in place heating by the solar radiation that is absorbed at depths, with the decay of absorbed radiation being shallow enough for negligible heat conduction to take place and the thermal waves do not travel and have a fixed phase.

The thermal waves profiles, such as the one shown in Figure 3.5, are used to extract the amplitude and phase of the thermal waves in each day. This is done by restricting the waves profile to the day of interest and fitting a sine wave to the timeseries of each thermistor. The amplitude and phase of the fitted sine waves are plotted as a function of depth for a number of selected days in Figures 3.6, 3.7 and 3.8. The phases plotted are in relation to the sun altitude, with a minimum altitude at midnight and a maximum altitude at midday (see Figure 3.4).

The variation in the amplitudes between the days is probably due to variation in cloud cover restricting the amount of solar radiation entering the sea ice. There is variation in the thermal wave structure between the days, such as the decay rate near the surface and transition depth between travelling and stationary thermal waves. The thermal waves in the multiyear sea ice appear a lot more consistent than those in first year sea ice, possibly due to consistent atmospheric conditions.

\subsection{Differential Equation for Thermal Waves}

Beginning with equation 2.1. we search for the role of the distributed heating term (and later an oscillating boundary condition) in the solution for the temperature.

$$
\rho(z, T) c(z, T) \frac{\partial T}{\partial t}=\frac{\partial}{\partial z}\left(k(z, T) \frac{\partial T}{\partial z}\right)+\frac{1}{2} P(z)\left(1+e^{i \omega t}\right) .
$$

We have introduced complex variables by denoting $T=\operatorname{Re}[\bar{T}]$ and dropping the over-bar for simplicity of analysing the periodic nature of the solar heating term. We have assumed a time dependence for the solar heating term of $\frac{1}{2}\left(1+e^{i \omega t}\right)$ such that the sun 'barely' sets at midnight and is directly overhead at midday, valid for an average November day in McMurdo Sound, Antarctica. This will be generalised to an arbitrary diurnal time dependence later. The equation is subject to a simplified Newton-style cooling version of the boundary conditions discussed in Chapter 2 (equation 2.5), as has been used by [19]. 

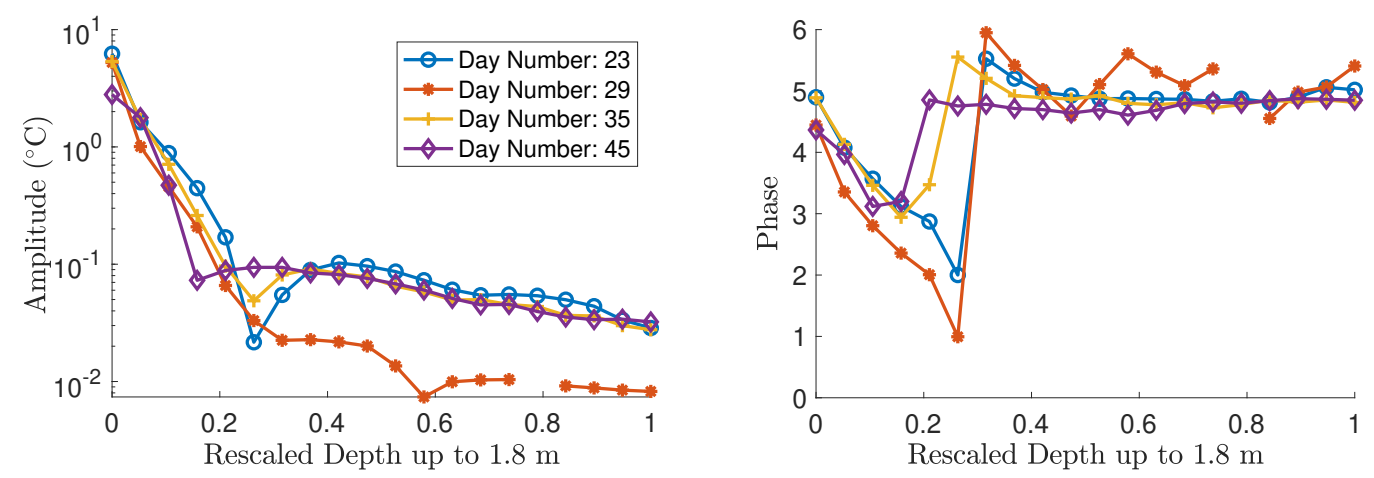

Figure 3.6: Thermal wave amplitude and phases from thermistor string AN1996 taken in first-year sea ice.
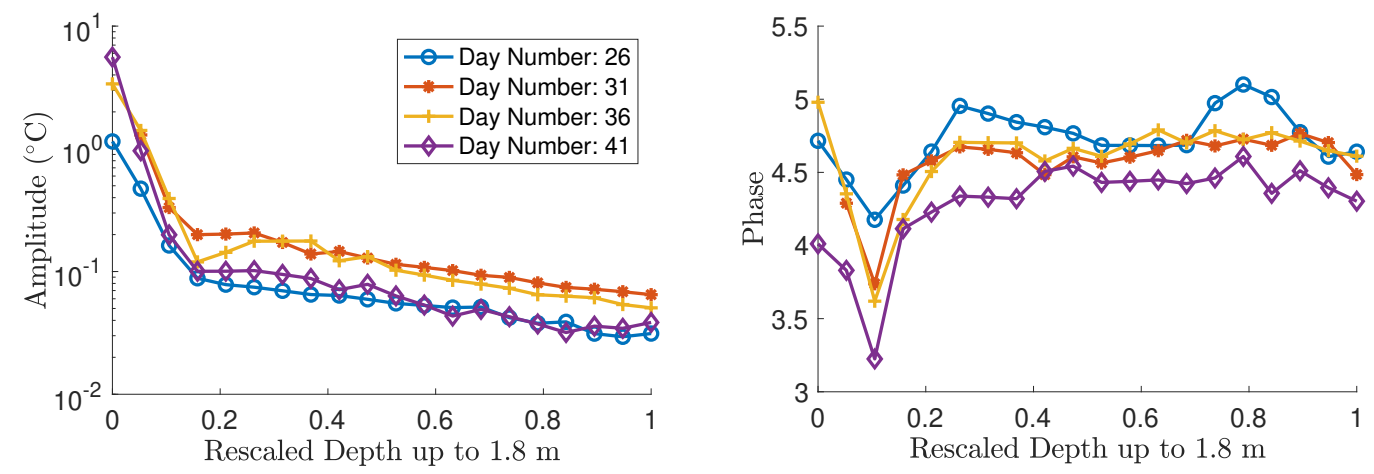

Figure 3.7: Thermal wave amplitude and phases from thermistor string AN1999 taken in first-year sea ice.
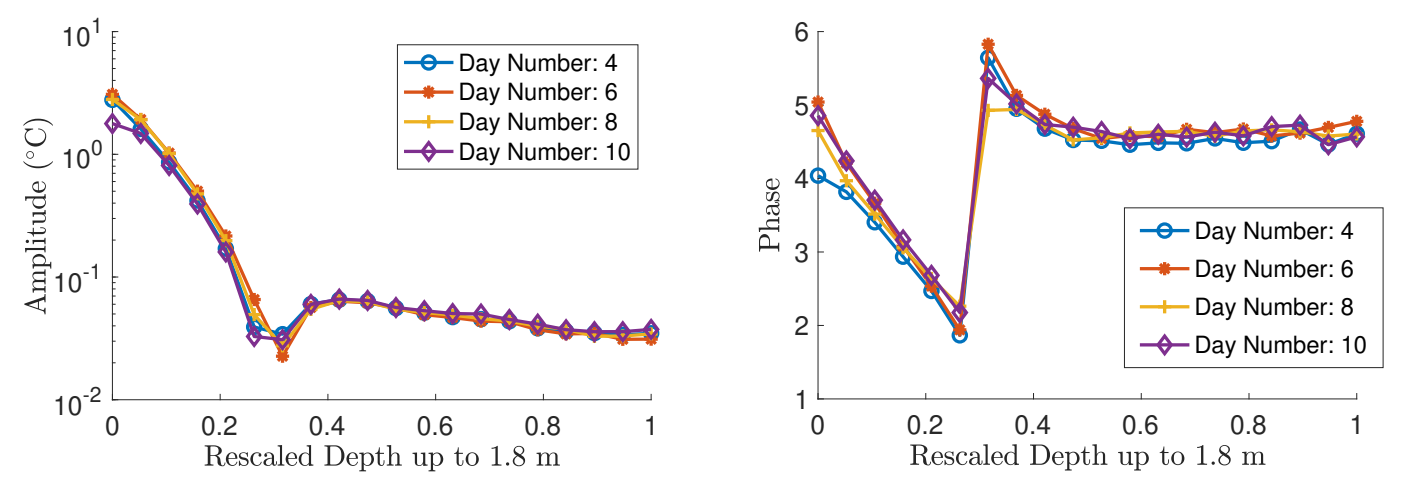

Figure 3.8: Thermal wave amplitude and phases from thermistor string AN2003 taken in multi-year sea ice. 


$$
\begin{aligned}
& T(H(t), t)=T_{\text {freezing }}, \\
& \left.\frac{\partial T(z, t)}{\partial z}\right|_{z=0}=\alpha\left[T(0, t)-T_{\text {air }}(t)\right], \alpha=\frac{h}{k},
\end{aligned}
$$

where $h$ is the heat transfer constant. This equation is strongly non-linear and due to the complicated analytical form of the physical parameters (see Appendix A), no simple analytical solutions can be found. The physical and thermal properties vary with temperature, depth and in principle also time due to the dynamic nature of the salinity and structure of the sea ice. Some analytical models begin by assuming the thermal conductivity is constant [18,66]. Over the temperature range from -25 to $-1.8{ }^{\circ} \mathrm{C}$ this assumption is valid to an accuracy of about $11 \%$. We do not consider this and will see the effect of neglecting the variation of the thermal conductivity on the thermal wave behaviour. By freezing the moving boundary with a Landau transformation [49] (as introduced in section 2.3), and re-arranging, we get

$$
\begin{aligned}
\frac{\partial T}{\partial t} & =\frac{1}{H^{2}(t) \rho c} \frac{\partial}{\partial z}\left(k(z, T) \frac{\partial T}{\partial z}\right)+\frac{1}{2} Q(z, T)\left(1+e^{i \omega t}\right)+z \frac{\dot{H}(t)}{H(t)} \frac{\partial T}{\partial z} \\
\dot{H}(t) & =\frac{1}{L \rho_{\text {ice }}}\left(\left.k \frac{\partial T}{\partial z}\right|_{z=1}-W_{\text {ocean }}\right)
\end{aligned}
$$

with $Q(z, T)=P(z) / \rho(T) c(T)$ and $H(t)$ is the thickness of the sea ice. The prefactors in the above equation are $k / \rho c \approx 10^{-6} \mathrm{~m}^{2} \mathrm{~s}^{-1}, Q_{0} \approx 10^{-3} \mathrm{Ks}^{-1}$ and $\dot{H} / H \approx 10^{-8} \mathrm{~s}^{-1}$ for sea ice of thickness $1 \mathrm{~m}$ (see Chapter 2) where the last term decreases for thicker ice later in the season. The last term on the right hand side of equation 3.3 is negligible and is neglected from here on.

Due to the non-linearity, the solution cannot be decomposed into a sum of terms, each satisfying the different driving forces (non-homogeneous boundary condition and distributed heating source). Taking a data driven analysis, from Figures 1.1 , 3.2 and 3.3 we see the sea ice and air temperatures may be decomposed into a sum of large, slow variations (on the scale of weeks and months), and smaller, quicker variations (on the scale of daily and subdaily). Using a two time analysis method [45] following the work of [66], we define fast and slow time as $t^{+}=t+\mathcal{O}\left(\epsilon^{2}\right), \tilde{t}=\epsilon t$ and model the air temperature as $T_{\text {air }}=\bar{T}_{\text {air }}(\tilde{t})+\epsilon \tilde{T}_{\text {air }}\left(t^{+}\right)$for a small parameter $\epsilon$. We have taken the distinguished limit [9] that the fast time oscillations are of order $\epsilon$. Further, we take the distinguished limit that the source term is of order $\epsilon$. Via the chain rule, the change of variables is $\frac{\partial}{\partial t}=\frac{\partial}{\partial t^{+}}+\epsilon \frac{\partial}{\partial t}$. 
Next we apply a regular perturbation expansion on the solution of the form $T\left(z, t^{+}, \tilde{t}\right)=T_{0}\left(z, t^{+}, \tilde{t}\right)+\epsilon T_{1}\left(z, t^{+}, \tilde{t}\right)+\mathcal{O}\left(\epsilon^{2}\right)$, and assume that each term is a bounded function of $t^{+}$. This allows for the parameters to be expanded in a Taylor series around $T=T_{0}$, given by

$$
k(z, T)=k\left(z, T_{0}\right)+\epsilon T_{1} k_{T}\left(z, T_{0}\right)+\mathcal{O}\left(\epsilon^{2}\right) \text { etc. },
$$

where subscript $T$ denotes partial differentiation with respect to temperature. From now on the brackets of the parameters evaluated at $\left(z, T_{0}\right)$ will be omitted. This gives the following for the differential equation, separated into powers of $\epsilon$.

$$
\begin{aligned}
\epsilon^{0}: \quad & \frac{\partial T_{0}}{\partial t^{+}}-\frac{1}{H^{2}(t) \rho c} \frac{\partial}{\partial z}\left(k \frac{\partial T_{0}}{\partial z}\right)=0, \\
\epsilon^{1}: \quad & \frac{\partial T_{0}}{\partial \tilde{t}}+\frac{\partial T_{1}}{\partial t^{+}}-\frac{1}{H^{2}(t) \rho c} \frac{\partial}{\partial z}\left(k \frac{\partial T_{1}}{\partial z}\right)-\frac{1}{2} Q\left(1+e^{i \omega_{0} t^{+}}\right) \\
& -T_{1}\left[D_{T} \frac{\partial^{2} T_{0}}{\partial z^{2}}+\left(\frac{k_{z}}{\rho c}\right)_{T} \frac{\partial T_{0}}{\partial z}+\frac{1}{2} Q_{T}\left(1+e^{i \omega_{0} t^{+}}\right)\right]=0 .
\end{aligned}
$$

where $D=\frac{k}{H^{2} \rho c}$. This is subject to the boundary conditions, separated into powers of $\epsilon$, as below.

$$
\begin{aligned}
z=0: & T_{0, z}=\alpha\left(T_{0}-\bar{T}_{\text {air }}(\tilde{t})\right), \\
& T_{1, z}=\alpha\left(T_{1}-\tilde{T}_{\text {air }}\left(t^{+}\right)\right), \\
& T_{n, z}=\alpha T_{n}, \quad n>1 . \\
z=1: & T_{0}=T_{\text {freezing }} \\
& T_{n}=0, \quad n>0 .
\end{aligned}
$$

with subscript $z$ indicating partial differentiation with respect to depth. The temperature derivatives of the parameters are all present in the coefficient of $T_{1}$ as the term in square brackets in equation 3.6. From Appendix A and typical values of $T_{0} \approx \frac{\partial T_{0}}{\partial z} \approx-10, \frac{\partial^{2} T_{0}}{\partial z^{2}} \approx 1$, estimated from thermistor array measurements (Figure 3.2), we see typical values of this coefficient are two orders of magnitude smaller than the other coefficients present in equation 3.6 and so the term with coefficient in square brackets is omitted in the remainder of the thesis. The distributed heating terms in the $\mathcal{O}\left(\epsilon^{1}\right)$ equation result in secular terms being present that cannot be eliminated. This indicates that the expansion is valid for a limited duration of 
fast time before the secular terms become significant. Equation 3.6 is separable and linear, allowing for the separation of the thermal waves of the three driving forces: the non-homogeneous boundary condition (thermal waves due to oscillating air temperatures), the distributed source term (thermal waves due to solar heating) and heating from the background temperature profile (non-oscillatory). We write $T_{1}=\theta_{1}+\theta_{2}+\theta_{3}$ with

$$
\begin{array}{lll} 
& \frac{\partial \theta_{1}}{\partial t^{+}}=\frac{1}{H^{2}(t) \rho c} \frac{\partial}{\partial z}\left(k \frac{\partial \theta_{1}}{\partial z}\right)+\frac{1}{2} Q e^{i \omega_{0} t^{+}}, \\
z=0 & \frac{\partial \theta_{1}}{\partial z}=\alpha \theta_{1}, \\
z=1 & \theta_{1}=0 . \\
& \frac{\partial \theta_{2}}{\partial t^{+}}=\frac{1}{H^{2}(t) \rho c} \frac{\partial}{\partial z}\left(k \frac{\partial \theta_{2}}{\partial z}\right), \\
z=0 & \frac{\partial \theta_{2}}{\partial z}=\alpha\left[\theta_{2}-F_{2}\left(t^{+}\right)\right], \\
z=1 & \theta_{2}=0 . \\
z=0 & \frac{\partial \theta_{3}}{\partial t^{+}}=\frac{1}{H^{2}(t) \rho c} \frac{\partial}{\partial z}\left(k \frac{\partial \theta_{3}}{\partial z}\right)+\frac{1}{2} Q-\frac{\partial T_{0}}{\partial \tilde{t}}, \\
z=1 & \frac{\partial \theta_{3}}{\partial z}=\alpha \theta_{3}, \\
\theta_{2}=0 .
\end{array}
$$

We focus on the component $\theta_{1}$ below (thermal waves generated by the solar heating) and treat $\theta_{2}$ (thermal waves generated by oscillating air temperatures) in a coming subsection. The component $\theta_{3}$ is of little physical interest, being the heating term due to the background temperature profile, hence is not analysed. The harmonic source term forces the time dependence of the solution to be harmonic, with $\theta_{1}=e^{i \omega_{0} t^{+}} Z(z ; \tilde{t})$, giving

$$
D Z^{\prime \prime}(z ; \tilde{t})+\frac{\partial k}{\partial z} \frac{1}{\rho c} Z^{\prime}(z ; \tilde{t})-i \omega_{0} Z(z ; \tilde{t})+\frac{1}{2} Q=0
$$

with $D=k /\left(H^{2}(t) \rho c\right)$ being the diffusivity and primes denote differentiation with respect to $z$ (depth). $Z$ is a function of depth and slow time as the coefficients depend on slow time. The explicit dependence on slow time will not be written in the remainder of this chapter.

Equation 3.10 is a second order, complex valued differential equation with variable coefficients and no closed form solution exists. It is convenient to reparameterize to eliminate the first derivative term [38], as well as write this in 
terms of non-dimensional functions. Our goal will become to find approximate solutions by applying a singular perturbation due to the presence of the small parameter and the numerically small value of the diffusivity. We let $Z=U(z) V(z)$ with

$$
\begin{aligned}
& U(z)=\exp \left(-\frac{1}{2} \int_{0}^{z} \frac{1}{k} \frac{\partial k}{\partial z} d x\right)=\sqrt{\frac{k(0)}{k(z)}} \\
& D V^{\prime \prime}(z)-\left(i \omega_{0}+D \frac{1}{2} \frac{\partial}{\partial z}\left(\frac{1}{k} \frac{\partial k}{\partial z}\right)+D \frac{1}{4}\left(\frac{1}{k} \frac{\partial k}{\partial z}\right)^{2}\right) V(z)+\frac{Q(z)}{2 U(z)}=0,
\end{aligned}
$$

and define the non-dimensional functions $f(z)=\frac{D(z)}{D(0)}, g(z)=\frac{Q(z)}{Q(0) U(z)}$ and further define $\delta=\frac{1}{\omega} D(0)$ (a small parameter) and $V(z)=\frac{1}{2 \omega_{0}} Q(0) W(z)$ to get

$$
\delta W^{\prime \prime}(z)-W(z)\left(\frac{i}{f(z)}+\frac{\delta}{2} \frac{\partial}{\partial z}\left(\frac{1}{k} \frac{\partial k}{\partial z}\right)+\frac{\delta}{4}\left(\frac{1}{k} \frac{\partial k}{\partial z}\right)^{2}\right)=-\frac{g(z)}{f(z)} .
$$

The coefficients of $W(z)$ that are of order $\delta$ are orders of magnitude smaller than 1 and so may be omitted in the leading order behaviour.

$$
\delta W^{\prime \prime}(z)-W(z) \frac{i}{f(z)}=-\frac{g(z)}{f(z)}
$$

subject to the boundary conditions

$$
\begin{array}{ll}
z=0: & W^{\prime}=W\left(\alpha+\frac{1}{k} \frac{\partial k}{\partial z}\right), \\
z=1: & W=0 .
\end{array}
$$

$W$ and $U$ are to be combined in the following manner to reproduce the complex valued temperature field. $\epsilon \theta_{1}=\frac{1}{2 \omega} Q(0) U(z) W(z) e^{i \omega t}$ where $\omega$ is the diurnal angular frequency.

In the remainder of this chapter we describe the terms present in equation 3.13 and show that a similar equation holds for $\theta_{2}$ (the thermal waves due to the oscillating air temperatures), indicating similar behaviour. Numerical solutions are presented along with data from thermistor string measurements and numerical simulations of the heat equation to motivate further analysis of approximate solutions to equation 3.13 which will be derived in the next chapter.

\subsubsection{Parameters}

The parameters in equations 3.13 and 3.11 that we need to quantify are the functions $g(z), f(z), k(z)$ and the value of $\delta$. 
The form of $g(z)$, the normalized source term, is obtained from photon-tracing Monte Carlo simulations presented by [69] as has been discussed earlier. The power absorbed per unit volume $P$ is parameterized as a decaying exponential with two decay constants in the two regions of sea ice.

$$
P(z)=P_{0} \times \begin{cases}e^{-\frac{z H}{v_{1}}} & , z \leq z_{c}, \\ e^{-\frac{z_{c} H}{v_{1}}} e^{-\frac{\left(z-z_{c}\right) H}{v_{2}}} & , z>z_{c} .\end{cases}
$$

$g(z)$ is given by

$$
g(z)=\tilde{g}(z) \times \begin{cases}e^{-\frac{z H}{v_{1}}} & , z \leq z_{c} \\ e^{-\frac{z_{c} H}{v_{1}}} e^{-\frac{\left(z-z_{c}\right) H}{v_{2}}} & , z>z_{c}\end{cases}
$$

with $\tilde{g}(z)=\frac{\rho\left(T_{0}(0)\right) c\left(T_{0}(0)\right)}{\rho\left(T_{0}(z)\right) c\left(T_{0}(z)\right) U(z)}$ and common values of the parameters are given in table 3.1 666, 69]. The Landau transformation (equation 3.3) has introduced the factors of $H$ in the exponent of $g(z)$; we re-parameterize the decay constants $\tilde{v}_{j}=v_{j} / H, j=1,2$ and drop the tilde on the $\tilde{v}_{j}$ from here on.

The term $\tilde{g}$ varies significantly only for large $z$, as seen in Figure 3.9 showing a particular form of $P$ normalized to have a maximum value of 1 , along with the forms of $g(z)$ for different surface temperatures.

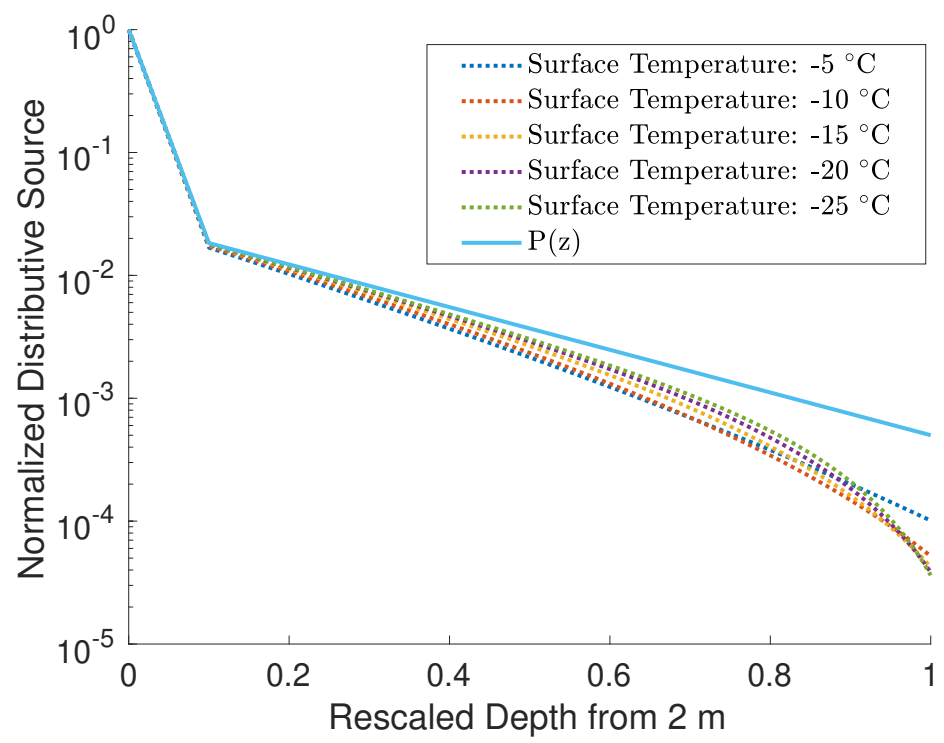

Figure 3.9: $P$ (solid line) and $g(z)$ (dotted lines) plotted after normalizing to have a maximum of 1 for a number of surface background temperatures, assuming a linear in depth profile for $T_{0}(z, \tilde{t})$. 
Incorporation of impurities such as algae in the ice may increase the power absorbed at deeper depths [69]. This can be modeled by adjusting the value of $v_{2}$ appropriately.

\begin{tabular}{|l|l|l|l|}
\hline Case & $v_{1}$ & $v_{2}$ & $z_{c} \times H$ \\
\hline $86 \mathrm{~b}$ & 0.054 & 0.3 & 0.24 \\
\hline $86 \mathrm{c}$ & 0.14 & 0.39 & 0.48 \\
\hline
\end{tabular}

Table 3.1: Fitted exponential form to the solar heating source term $P(z)$ attained with Monte Carlo scattering simulations from [69]. H is the sea ice thickness in meters.

The form of $f(z)$ and $k(z)$ is obtained from general diffusivity and conductivity curves. The formulae for the physical parameters, and hence diffusivity, are provided in Appendix $\mathrm{A}$, and depend on the background temperature and salinity profiles. The temperature profile changes as weather systems diffuse through the ice and hence change the form of $f(z)$ and $k(z)$. Figure 3.10 shows the normalized diffusivity $f(z)$ assuming linear background temperature profiles for various surface temperatures, along with an average fitted quadratic being $f_{\text {quad }}(z)=1-0.42 z-0.48 z^{2} . k(z)$ is also shown for various surface temperatures and is fitted with a linear line for $z<0.8$ for each surface temperature, $k(z) / k(0)=1+a z$. The values of $a$ of the fitted lines are listed in table 3.2. The diffusivity of a substance is never zero and so $f(z)$ never reaches zero on the interval $z \in[0,1]$.

The value of $\delta$ is $\delta=\frac{D(0)}{\omega}=\frac{60 * 60 * 24}{2 \pi} D(0)$. We summarize the values of $\delta$ based on the temperature at the surface of the ice. Note that the value of $\delta$ will depend on the thickness of the ice so we tabulate $\delta H^{2}$, as $D(0)=k /\left.\left(H^{2} \rho c\right)\right|_{z=0}$. The tabulated values are for a surface salinity of 5 ppt and only vary slightly for salinities between 2 ppt and 10 ppt.

\subsubsection{Oscillatory Air Temperatures}

The thermal waves generated by the oscillatory air temperatures are modelled to leading order by equation 3.8 , repeated below

$$
\begin{aligned}
& \frac{\partial \theta_{2}}{\partial t^{+}}=\frac{1}{H^{2}(t) \rho c} \frac{\partial}{\partial z}\left[k \frac{\partial \theta_{2}}{\partial z}\right], \\
z=0: & \frac{\partial \theta_{2}}{\partial z}=\alpha\left[\theta_{2}-\tilde{T}_{\text {air }}\left(t^{+}\right)\right], \\
z=1: & \theta_{2}=0 .
\end{aligned}
$$



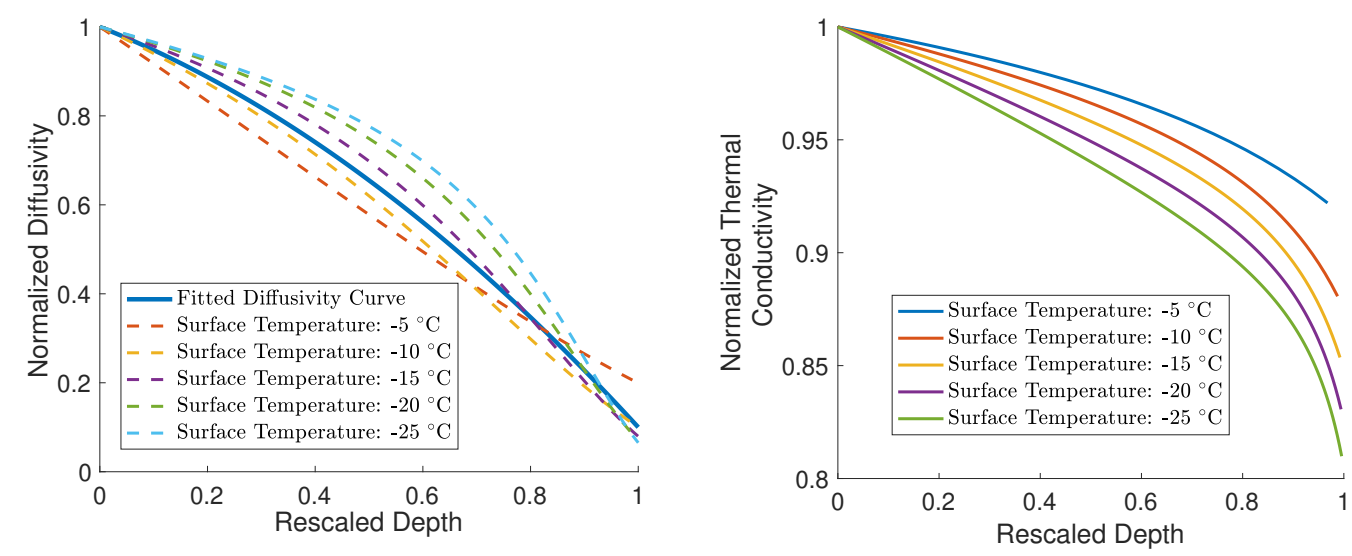

Figure 3.10: Diffusivity (left) and thermal conductivity (right) as a function of depth assuming a linear temperature profile for various surface temperatures. The diffusivity and thermal conductivity have been normalized to their maximum value. These plots are for a constant salinity of 5 pt and do not vary significantly for salinities between 2 ppt and 10 ppt

\begin{tabular}{|l|l|l|}
\hline$\left.T_{0}\right|_{z=0}$ & $\delta H^{2}$ & $a$ \\
\hline-5 & $5.72 \times 10^{-3}$ & -0.064 \\
\hline-10 & $1.14 \times 10^{-2}$ & -0.081 \\
\hline-15 & $1.43 \times 10^{-2}$ & -0.096 \\
\hline-20 & $1.61 \times 10^{-2}$ & -0.112 \\
\hline-25 & $1.75 \times 10^{-2}$ & -0.129 \\
\hline
\end{tabular}

Table 3.2: The values of the small parameter $\delta$ and the fitted depth variation of the thermal conductivity for $z<0.8, k(z) / k(0)=1+a z$, for various ice surface temperatures in ${ }^{\circ} \mathrm{C}$. $H$ is the sea ice thickness in meters. 
This is a standard heat equation with non-constant coefficients, and so we separate the variables in a similar fashion to the method done for $\theta_{1}$. We first use a Fourier sum to represent the fast time air temperatures, $\tilde{T}_{\text {air }}\left(t^{+}\right)=\sum A_{n} e^{i \omega_{n} t^{+}}$. Due to the linearity of the above equation in $\theta_{2}$, we form the Ansatz $\theta_{2}=\sum Z_{n}(z) e^{i \omega_{n} t^{+}}$ with

$$
\begin{array}{ll} 
& i \omega_{n} Z_{n}=\frac{1}{H^{2} \rho c} \frac{d}{d z}\left(k \frac{d Z_{n}}{d z}\right), \\
z=0: & \frac{d Z_{n}}{d z}=\alpha\left[Z_{n}-A_{n}\right], \\
z=1: & Z_{n}=0 .
\end{array}
$$

For each component $Z_{n}$, we apply the same methodology as was applied between equations 3.7 and 3.13 . Letting $Z_{n}(z)=U(z) V_{n}(z)$ with $U(z)=\sqrt{\frac{k(0)}{k(z)}}$ we get

$$
D V_{n}^{\prime \prime}(z)-\left(i \omega_{n}+D \frac{1}{2} \frac{\partial}{\partial z}\left(\frac{1}{z} \frac{\partial k}{\partial z}\right)+D \frac{1}{4}\left(\frac{1}{k} \frac{\partial k}{\partial z}\right)^{2}\right) V_{n}(z)=0
$$

By re-parameterizing with $f(z)=\frac{D(z)}{D(0)}, \delta=\frac{1}{\omega_{n}} D(0)$ and $W_{n}=V_{n}$ and neglecting the small terms in the coefficient of $V_{n}$ to get

$$
\delta W_{n}^{\prime \prime}(z)-\frac{i}{f(z)} W_{n}(z)=0
$$

along with the boundary conditions

$$
\begin{aligned}
& W_{n}^{\prime}(0)=\alpha\left(W_{n}(0)-A_{n}\right), \\
& W_{n}(1)=0
\end{aligned}
$$

This is the same equation as equation 3.13 but without the source term and with a non-homogeneous boundary condition. Due to the depth varying coefficients it cannot be solved via elementary methods and so approximate solutions will be considered in the next chapter.

\subsubsection{Non-constant Conductivity Terms}

Most models of sea ice thermodynamics neglect the term $k_{z} T_{z}$ from the heat equation, assuming that $k$ does not depend on depths [18,66]. This assumption is valid 
when $\frac{\partial k}{\partial z} \frac{\partial T}{\partial z} \ll k(z, T) \frac{\partial^{2} T}{\partial z^{2}}$. This is supported by the thermistor string data, Figure 3.11 shows the contribution ratio of the term $\frac{\partial k(z, T)}{\partial z} \frac{\partial T}{\partial z}$ relative to $k(z, T) \frac{\partial^{2} T}{\partial z^{2}}$ for the 1996 thermistor string.
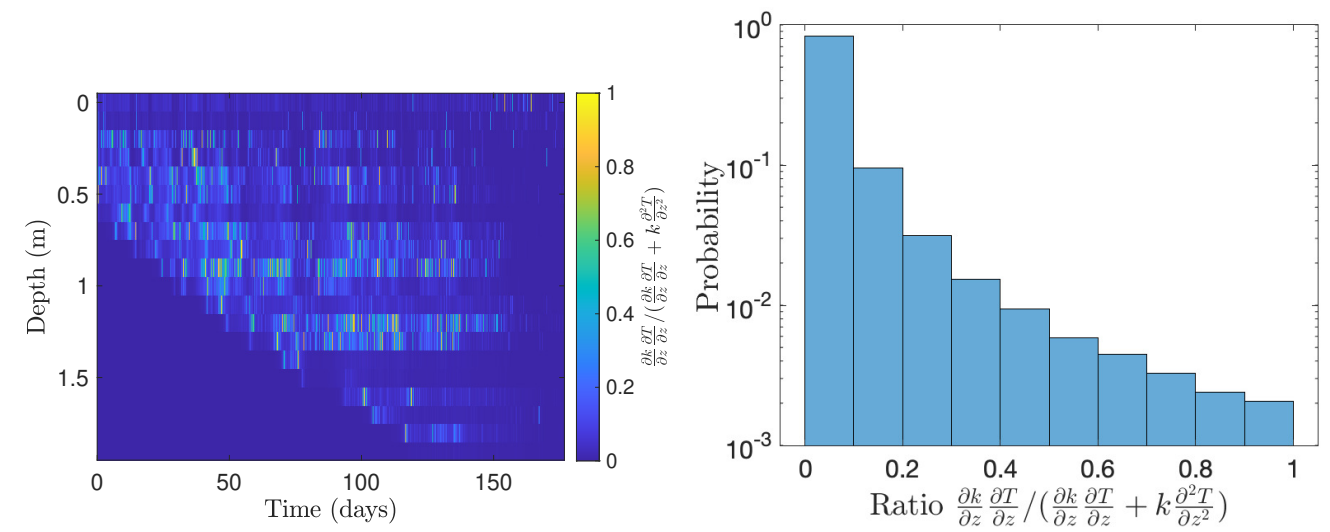

Figure 3.11: From the 1996 thermistor string data, the ratio $\frac{\frac{\partial k}{\partial z} \frac{\partial T}{\partial z}}{\frac{\partial k}{\partial z} \frac{\partial T}{\partial z}+k \frac{\partial^{2} T}{\partial z^{2}}}$ is presented. Points in the water were ignored. Data from different years resulted in similar results. Left: The ratio is plotted assuming a salinity of 5ppt. Near the surface and later in the season the ratio is seen to be smaller than in other regions on average. Right: Histogram of occurrence of the term ratio, with a logarithmic scale on the y-axis. This ratio is bigger than 0.5 for $1.8 \%$ of the points, and bigger than 0.1 for about $17 \%$ of the points. The average ratio observed was 0.07 .

We see that in our analysis, the term $k_{z}$ does not show up in the leading order behaviour of $W(z)$. It does appear in equation 3.11, and leads to the modification of the amplitude of the thermal waves by the factor $\sqrt{\frac{k(0)}{k(z)}}$. For sea ice, the thermal conductivity may vary by about $11 \%$ over the depth of the sea ice. Omission of this term miscalculates the amplitude of the thermal wave by about $5 \%$.

\subsection{Numerical and Data Comparison}

Figure 3.12 shows numerical simulations (using the tool developed in Chapter 2) with various source term sizes, $P_{0}=P(0)$, and various surface temperatures. We see that the effects of $P_{0}$ on the thermal wave behaviour are insignificant while the effects of the surface temperature are extremely significant, modelled with varying the parameter $\delta$ appropriately. Further comparisons between these numerical simulations and solutions to equation 3.10 show good agreement. Examples of this are presented in Figure 3.13. 

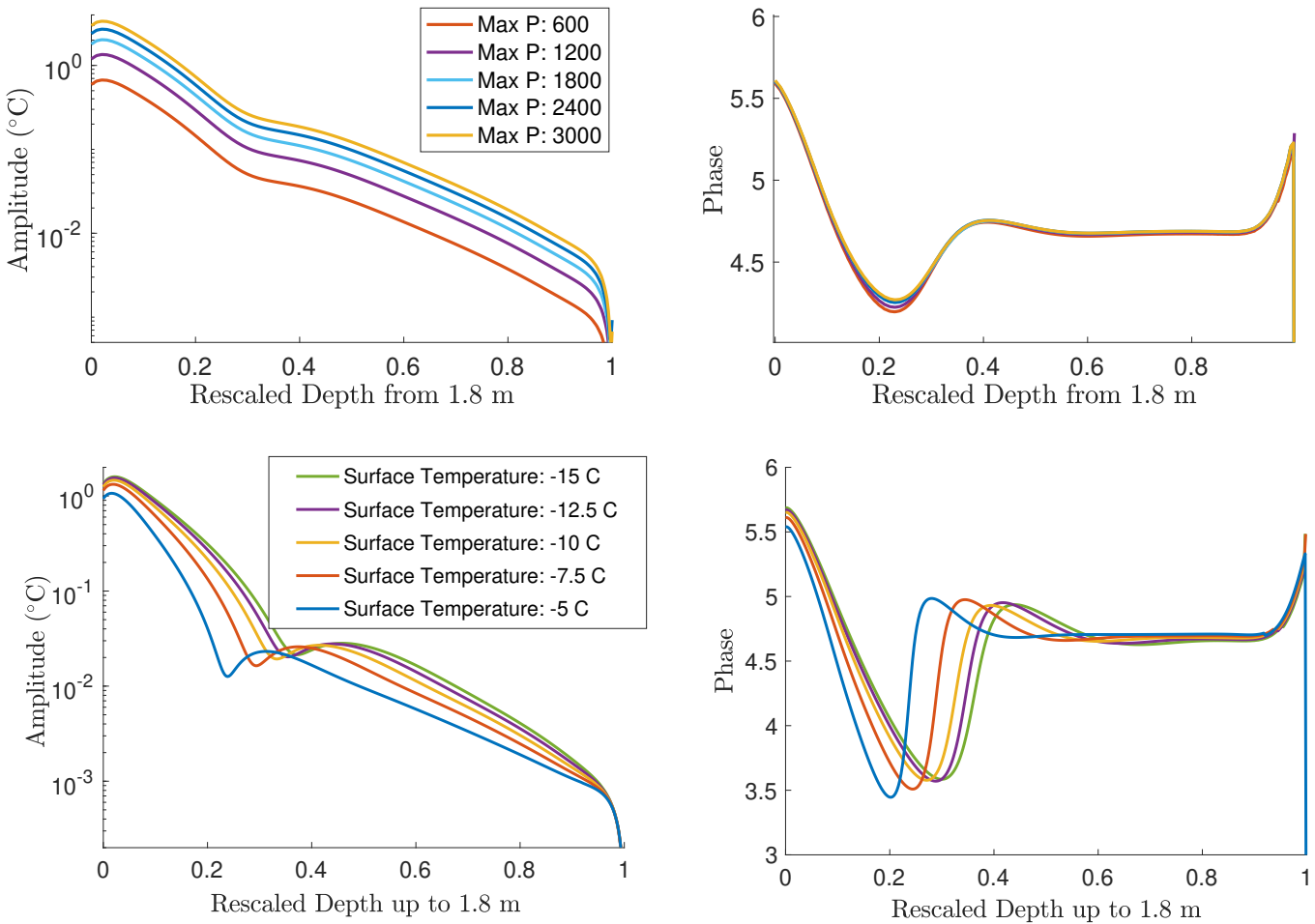

Figure 3.12: Thermal waves analysis for various values of $P_{0}$ and surface temperatures from numerical simulations (Chapter 2). Amplitude is plotted on the left and phase on the right. The distributed source parameters used are: $v_{1}=0.05 \mathrm{~m}, v_{2}=0.5 \mathrm{~m}, z_{c}=0.2 \mathrm{~m}$, $\alpha=10 \mathrm{~m}^{-1}$. Top: A constant surface temperature of $-10^{\circ} \mathrm{C}$ is used and $P_{0}$ is varied. As seen the amplitudes of the thermal waves scale with the value of $P_{0}$ but the thermal wave structure remains relatively unchanged. Bottom: Variation of the surface temperature from $-5^{\circ} \mathrm{C}$ to $-15^{\circ} \mathrm{C}$ with a constant value of $P_{0}=3000 \mathrm{~J} / \mathrm{m}^{3}$.
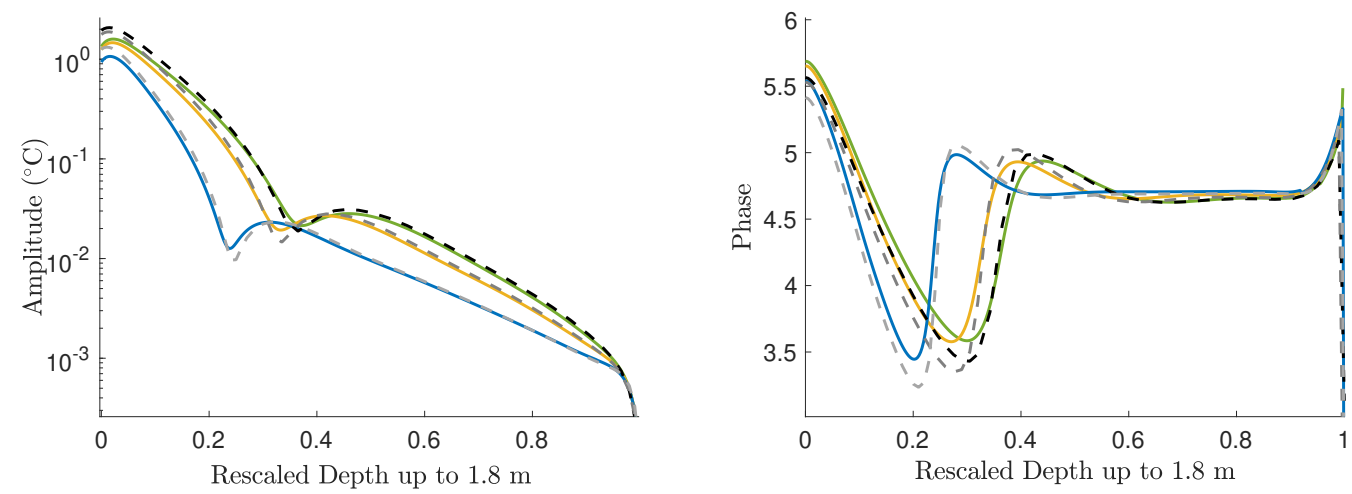

Figure 3.13: Thermal waves from the numerical simulations (Chapter 2) in the bottom of Figure 3.12 (solid lines) and numerical solutions of equation 3.10 (dashed lines) of the same parameters. Amplitudes on the left and phases on the right. 
Including an oscillatory air temperature of amplitude $2{ }^{\circ} \mathrm{C}$ and lagging the solar heating term by $\frac{\pi}{2}$, a similar comparison is achieved in Figure 3.14 , comparing the numerical simulation results to the numerical solution of equations 3.10 and 3.17
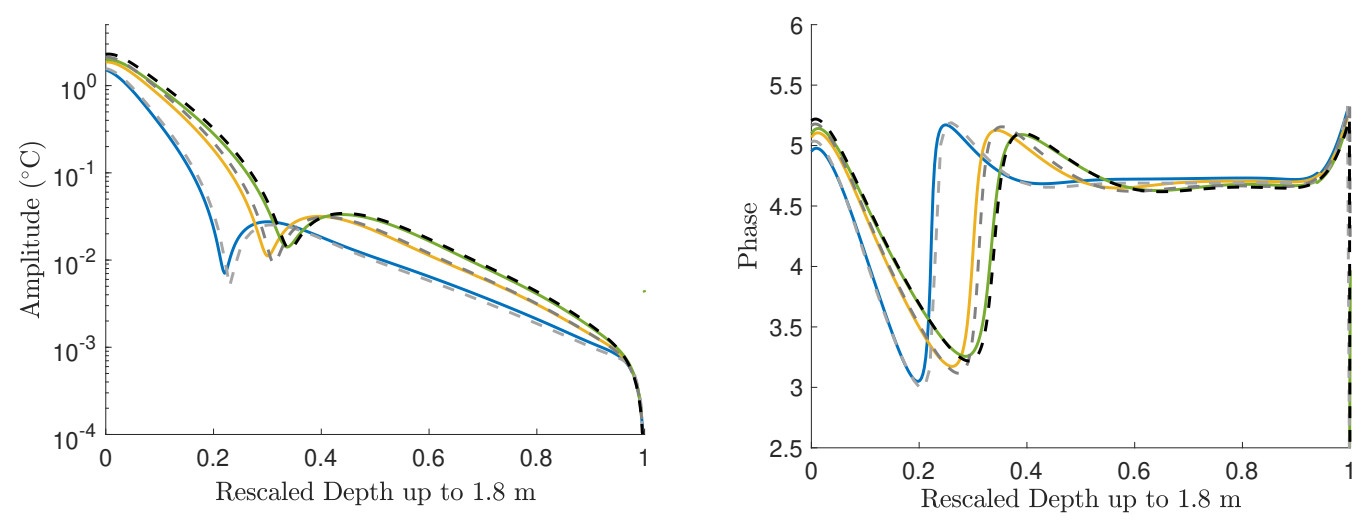

Figure 3.14: Thermal waves due to solar heating and oscillatory air temperatures. Plotted are numerical simulations as in Figure 3.12 with an added oscillating air temperature of amplitude $2{ }^{\circ} \mathrm{C}$ and lagging the solar heating term by $\frac{\pi}{2}$ (solid lines), and numerical solutions of equations 3.10 and 3.17 (dashed lines). Amplitudes on the left and phases on the right.

The numerical solutions of equations 3.10 and 3.17 appear to posses the same features as the data analysis presented in Figures 3.6, 3.7 and 3.8. Numerical solutions of equation 3.10 and 3.17 are overlaid on the data analysis from the 1996 thermistor in Figure 3.15. The simulation parameters used where: $P_{0}=$ $3000 \mathrm{~J} / \mathrm{m}^{3}, \alpha=10 \mathrm{~m}^{-1}, H=1.8 \mathrm{~m}, z_{c}=0.2 \mathrm{~m}, v_{1}=0.05 \mathrm{~m}, T_{s}=-5{ }^{\circ} \mathrm{C}$ (the surface temperature) and the air temperature oscillations are of amplitude $2{ }^{\circ} \mathrm{C}$ and lagging the solar heating by $\frac{\pi}{2}$. The plotted lines have the following parameters. Dotted line: $v_{2}=0.5 \mathrm{~m}$. Dashed line: $v_{2}=2 \mathrm{~m}$.

When simulated with a value for $v_{2}$ of 2 rather than 0.5 , the standard value for sea ice [69], a better match with the data is obtained for the decay of the thermal waves for $z>0.3$. This is probably due to increased impurity concentrations at lower depths, as studied by [69].

It has been noted previously [11, 66] that the numerical solutions exhibit a solid-state greenhouse effect with the maximum amplitude of the thermal wave being just below the surface, as seen in our numerical calculations, but missing in the data. This is physically due to the surface losing heat to the atmosphere, cooling it with respect to the layer of sea ice below it. It is not observed in the data as the $0.1 \mathrm{~m}$ spacing of the thermistors is too coarse to resolve it, and furthermore 

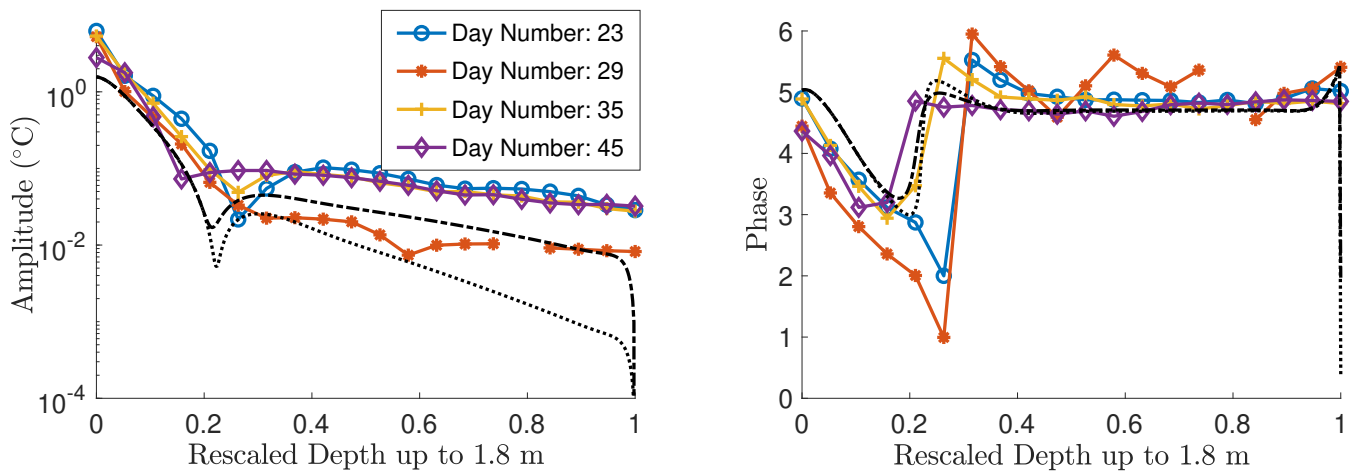

Figure 3.15: Numerical solutions to equation 3.10 and 3.17 (solid and dashed black lines) with parameters as described in the text, overlaid with thermal wave amplitude and phase from the 1996 thermistor data (Figure 3.6.).

travelling waves due to oscillating air temperatures drown out this effect at the surface (see Figures 3.14 and 3.15.

The numerical solutions of the above theory and simulations are seen to be in close agreement and posses the same features as the data. In the next chapter we seek analytical approximations to the solutions of equations 3.13 and 3.18 . 


\section{Chapter 4}

\section{Mathematical Approximations}

In this chapter we analyse solutions to the heat diffusion equation derived in the previous chapter. Equations 3.13 and 3.18 are (non)-homogeneous second order ordinary differential equations with varying coefficients and cannot be solved using elementary methods. Numerical solutions presented in the previous chapter have highlighted a number of interesting behaviours, including:

- The travelling thermal waves at shallow depths,

- The stationary thermal waves at deeper depths,

- The change over region between the two wave-types,

- The solid state green-house effect near the surface of the ice.

Previous analysis of solar heating [18, 53, 108] has assumed constant thermal and optical properties. From our analysis it will become apparent that it is vital to consider the variation in the optical properties to understand the behaviour of the thermal waves, and considerations of the variation of the thermal properties gives further refinements to the wave propagation behaviour.

In this chapter we will begin by introducing the techniques of boundary layer theory. Motivated by the small parameters present in equations 3.13 and 3.18 we apply boundary layer theory to derive approximate solutions to these equations. We will find inner and outer solutions to equation 3.13 . followed by the leading order WKB approximation to equations 3.13 and 3.18, giving a uniform approximation to each. The WKB approximation to equation 3.13 is approximated in terms of elementary functions using the Method of Steepest Descent. Each of the above is compared with numerical examples. 


\subsection{Introduction}

Here we introduce some mathematical techniques following [9] that will be used in the forthcoming chapter. The differential equations of interest, equations 3.13 and 3.18, have small parameters multiplying the highest derivative term. In the limit $\delta \rightarrow 0$, this is a singular perturbation to the differential equation as the order of the differential equation changes between the differential equation with and without a zero value of $\delta$.

\subsubsection{Matched Asymptotics}

Matched asymptotic analysis is a powerful technique to analyse the solution behaviour of a differential equation with a small parameter. The core to these methods is to identify regions where the solution to the differential equation is slowly varying (outer regions), and regions/points where the solution is rapidly varying as the small parameter, $\delta$, is varied (boundary layers or inner regions). The width of boundary layers decreases as the small parameter is decreased, and the solution becomes discontinuous in the boundary layer in the limit $\delta \rightarrow 0$. In order to apply this method we need to know where the boundary layers are.

In the outer regions higher order derivative terms with coefficient terms of small parameters become negligible. The solution is approximated by a solution to a similar differential equation with $\delta=0$. The solution to this equation is called the outer solution.

Within inner regions, the narrow width of the boundary layer(s) allows for suitable approximations to be made to the differential equation and an approximate solution to the differential equation is obtained, valid in the boundary layer, called the inner solution. The outer and inner solution are then matched to determine any previously undetermined coefficients by comparing the solutions over a common region of validity which in the limit $\delta \rightarrow 0$ covers the entire ice depth.

\subsubsection{WKB Theory}

Unlike matched asymptotics where solutions are approximated over subsets of the interval of interest, a WKB approximation gives a uniform approximation over the entire interval. The key to the WKB approximation is to expand the solution in the form of an exponential with various 'sizes' of phase functions. The 'size' is determined by the power of the small parameter present in the differential equation. As with matched asymptotics, the solution is valid in the limit of the 
small parameter tending to zero. The WKB expansion of second order differential equations has been studied thoroughly and we present a derivation of it here.

We begin with a homogeneous second order equation with small parameter $\epsilon$.

$$
\epsilon^{2} W^{\prime \prime}(z)-Q(z) W(z)=0
$$

We write the solution in terms of an exponential with a varying amplitude and phase function

$$
W(z)=A(z) e^{S(z) / \delta}
$$

where $\delta$ is a small parameter that characterizes the solution's quick oscillations or exponential behaviour in response to the small parameter in the differential equation. By expanding $A(z)$ and $S(z)$ as power series of $\delta$ the two expansions are combined into a single exponential expansion and written as

$$
W(z) \sim \exp \left(\frac{1}{\delta} \sum_{n=0}^{\infty} \delta^{n} S_{n}(z)\right), \delta \rightarrow 0
$$

where the functions $S_{n}(z)$ characterize the solution and are $\mathcal{O}(1)$. We substitute this form into equation 4.1 by first calculating $W^{\prime}(z)$ and $W^{\prime \prime}(z)$.

$$
\begin{array}{lll}
W^{\prime}(z) \sim\left(\frac{1}{\delta} \sum_{n=0}^{\infty} \delta^{n} S_{n}^{\prime}(z)\right) \exp \left(\frac{1}{\delta} \sum_{n=0}^{\infty} \delta^{n} S_{n}(z)\right) & , \delta \rightarrow 0, \\
W^{\prime \prime}(z) \sim\left[\left(\frac{1}{\delta} \sum_{n=0}^{\infty} \delta^{n} S_{n}^{\prime}(z)\right)^{2}+\left(\frac{1}{\delta} \sum_{n=0}^{\infty} \delta^{n} S_{n}^{\prime \prime}(z)\right)\right] \exp \left(\frac{1}{\delta} \sum_{n=0}^{\infty} \delta^{n} S_{n}(z)\right) & , \delta \rightarrow 0 .
\end{array}
$$

Substituting in to equation 4.1 and dividing off the exponential term we get

$$
\epsilon^{2}\left[\left(\frac{1}{\delta} \sum_{n=0}^{\infty} \delta^{n} S_{n}^{\prime}(z)\right)^{2}+\left(\frac{1}{\delta} \sum_{n=0}^{\infty} \delta^{n} S_{n}^{\prime \prime}(z)\right)\right]=Q(z)
$$

The largest term on the left hand side is $\frac{\epsilon^{2}}{\delta^{2}} S_{0}^{\prime}(z)$ and is chosen to be same order of magnitude as $Q(z)$ (assuming $Q(z) \neq 0$ ) with the distinguished limit $\delta=\epsilon$. Since the terms $S_{n}(z)$ are all $\mathcal{O}(1)$, we match powers of $\epsilon$ on the left and right hand sides of the equation to get the following series of equations for the $S_{n}(z)^{\prime}$ 's

$$
\begin{aligned}
S_{0}^{\prime 2}(z) & =Q(z), \\
2 S_{0}^{\prime}(z) S_{1}^{\prime}(z)+S_{0}^{\prime \prime}(z) & =0, \\
2 S_{0}^{\prime}(z) S_{n}^{\prime}(z)+S_{n-1}^{\prime \prime}+\sum_{i=1}^{n-1} S_{i}^{\prime}(z) S_{n-i}^{\prime}(z) & =0, \quad n \geq 2 .
\end{aligned}
$$


These equations can be solved iteratively with the first three solutions shown below

$$
\begin{aligned}
& S_{0}(z)= \pm \int_{0}^{z} \sqrt{Q(x)} d x, \\
& S_{1}(z)=-\frac{1}{4} \ln (Q(z)), \\
& S_{2}(z)= \pm \int_{0}^{z}\left(\frac{Q^{\prime \prime}(x)}{8 Q^{3 / 2}(x)}-\frac{5\left(Q^{\prime}(x)\right)^{2}}{32 Q^{5 / 2}(x)}\right) d x .
\end{aligned}
$$

Combining the first two terms gives a uniformly valid approximation over the entire interval (that $Q(z) \neq 0$ ) up to order $\epsilon$. Further terms can be included to attain more accurate approximations, however it is important to note that the series is only valid in the limit $\epsilon \rightarrow 0$ and for a fixed $\epsilon$ the WKB series with increasing number of terms typically diverges. For a terminated series to be a good approximation for the solution we require that every successive term in the terminated series be significantly smaller than the previous. Further, we require that the next term in the series is sufficiently smaller than 1 . So for a terminated series of the first two terms to be a good approximation, $W(z) \sim \exp \left(\frac{1}{\epsilon} S_{0}(z)+S_{1}(z)\right)$, we require $\frac{1}{\epsilon} S_{0}(z) \gg S_{1}(z)$ and $\epsilon S_{2}(z) \ll 1$ for all $z$ in the interval of interest.

\subsubsection{Laplace's Method}

Laplace's method is used to approximate integrals of an exponential with a large parameter by taking the limit as the large parameter $x$ tends to infinity. A Laplace integral is of the form

$$
I=\int_{a}^{b} e^{x \phi(s)} f(s) d s,
$$

(note: $f(s)$ is a general function here) where $\phi$ is a real valued function. In the limit that $x \rightarrow \infty$ most of the contribution to the integral comes from a narrow region around the maximum point of $\phi(s)$. Here we will treat the case that this occurs at one of the end points. The integral may be approximated by the integral over a small narrow region around the endpoint where a maximum in $\phi$ is attained.

We introduce the integral

$$
I_{\epsilon}=\int_{b-\epsilon}^{b} e^{x \phi(s)} f(s) d s,
$$

where we have assumed that the maximum of $\phi(s)$ is attained at the point $s=b$. As is shown by [9] the integral that we have neglected, with integration limits 
from $a$ to $b-\epsilon$, is subdominant to $I_{\epsilon}$ in the limit $x \rightarrow \infty$. To approximate the integral $I_{\epsilon}$ we note that the integral is over an infinitesimal region and so the functions $f(s)$ and $\phi(s)$ are well approximated by their Taylor series. That is, in the region $b-\epsilon<s<b$ we have

$$
\begin{aligned}
f(s) & \approx f(b)+\ldots \\
\phi(s) & \approx \phi(b)+(s-b) \phi^{\prime}(b)+\ldots
\end{aligned}
$$

Substituting this into the integral $I_{\epsilon}$ we get the leading order behaviour of the integral being

$$
I_{\epsilon} \sim \int_{b-\epsilon}^{b} e^{x\left(\phi(b)+(s-b) \phi^{\prime}(b)\right)} f(b) d s, \quad x \rightarrow \infty .
$$

This integral can be evaluated! However we note that the leading order behaviour should not depend on $\epsilon$, the length of the interval where the integrand is largest, and extending the bounds of integration to $-\infty$ introduces only exponentially small errors in the limit $x \rightarrow \infty$. The approximation of the integral $I_{\epsilon}$ becomes

$$
I_{\epsilon} \sim \int_{-\infty}^{b} e^{x\left(\phi(b)+(s-b) \phi^{\prime}(b)\right)} f(b) d s \sim \frac{1}{x} f(b) e^{x \phi(b)} \frac{1}{\phi^{\prime}(b)}, \quad x \rightarrow \infty .
$$

For an integral where the bounds are closer together than the decay of the exponential, for example, if $|a-b| \sim \frac{1}{x}$ then this approximation becomes invalid.

\subsubsection{Method of Steepest Descent}

The method of steepest descent applies to integrals with exponential terms with large parameters and complex phase functions,

$$
I=\int_{C} e^{x \phi(s)} f(s) d s,
$$

where $C$ is a complex contour of integration, $\phi(s), f(s)$ are analytic functions of $s$ and $x$ is a large parameter. The core to this method is to use the analyticity of the integrand to deform the contour of integration to a contour of steepest descent/ascent. General complex variable theory [9,22] shows that contours of constant imaginary component are also contours of steepest descent/ascent of the real component, and vice versa. Further, so long as we don't pass branch cuts or through branch points, the contour of integration may be deformed to a different contour with the same beginning and end points. We deform the original contour of integration to a set of contours of constant imaginary component of the phase 
function, $\phi(s)$, from either endpoint of the original contour and join these with a contour of constant real component of $\phi(s)$. Along each of the first two contours of integration the integral is a Laplace integral as the exponential function takes on only real values, and further attains its maximum at one end of the integration contour (unless a saddle point is present, these are dealt with in [9]). The contour of constant real component is usually sent to infinity where the real component of $\phi(s)$ tends to $-\infty$ and so the integrand vanishes along this contour.

\subsection{Boundary Layer Analysis}

We begin with asymptotic analysis of equation 3.13, reprinted below, following the work presented in [69].

$$
\delta W^{\prime \prime}(z)-W(z) \frac{i}{f(z)}=-\frac{g(z)}{f(z)} .
$$

The small parameter in the coefficient of the second derivative term induces boundary layers near $z=0$ and $z=1$ as may be see by the numerical solutions (see, for instance, Figure 4.1). Far away from these points the solution takes on the form of the outer solution, and near these points more complex behaviour is present.

\subsubsection{Matched Asymptotics}

The leading order outer solution is found by setting $\delta=0$ in equation 4.5 . Here we immediately retrieve the outer solution as

$$
W_{\text {outer }}(z)=-i g(z) .
$$

The outer solution takes the form of stationary waves decaying at the same rate as the source term. This can be seen more explicitly by writing down the equivalent temperature profile oscillations described by the outer solution.

$$
\epsilon \theta_{1, \text { outer }}=-\frac{Q(z)}{2 \omega} e^{i\left(\omega_{0} t^{+}+\pi / 2\right)} .
$$

This is interpreted as the in-place heating due to the source term, where conduction is negligible. The temperature evolution is out of phase from the heating source by $\frac{\pi}{2}$, due to the ice retaining heat. The outer solution is not self consistent when $g(z)$ varies as $e^{-\frac{z}{\sqrt{\delta}}}$ or steeper, as then the second derivative term in equation 3.13 is of order 1 like the source term.

We turn to the inner region and focus on the boundary layer at $z=0$. We define the inner variable $Z=\frac{z}{\sqrt{\delta}}$. The functions $f(\sqrt{\delta} Z)$ and $g(\sqrt{\delta} Z)$ are expanded 
in a Maclaurin series, and to leading order in $\sqrt{\delta}$ we retain only the constant term being $f(0)=g(0)=1$. Within the inner region we wish to solve

$$
W_{\text {inner }}^{\prime \prime}(Z)-i W_{\text {inner }}(Z)=-1
$$

This is exactly solve-able [28], and solutions may be written as a sum of homogeneous and particular solutions.

$$
W_{\text {inner }}(Z)=c_{1} \exp (\sqrt{i} Z)+c_{2} \exp (-\sqrt{i} Z)-i
$$

In order to match with the outer solution, we compute the following limits,

$$
\begin{aligned}
& \lim _{z \rightarrow 0} W_{\text {outer }}=-i, \\
& \lim _{Z \rightarrow \infty} W_{\text {inner }}=c_{1} \exp (\sqrt{i} Z)-i,
\end{aligned}
$$

and see that we must choose $c_{1}=0$ to match the two expressions. The coefficient $c_{2}$ is determined by the boundary conditions at $z=0: \frac{1}{\sqrt{\delta}} \frac{d W}{d Z}=\left(\alpha+\frac{k_{z}}{k}\right) W$. However, expanding this boundary condition in $\sqrt{\delta}$ we get $c_{2}=0$, and it appears we have lost the structure of the boundary layer to leading order. Further, we note that for physically relevant parameters, the outer solution is inconsistent for $z<z_{c}$, as $v_{1} \sim \sqrt{\delta}$, being the source term decay parameter. The inner solution does not appear to posses the structure of the travelling thermal waves due to the quick decay of the source term or the interaction with the atmosphere via the presence of the term $\alpha W$ in the upper boundary condition. This is due to the above form being derived in the limit $\delta \rightarrow 0$ with all other parameters assumed constant with respect to $\delta$. However, physically relevant parameter values do match significant power of $\delta$, with $\sqrt{\delta} \approx 0.1$. In order to better model the interaction with the atmosphere and the source term, we take a number of distinguished limits. These are motivated by the physically relevant values of $v_{1} \approx 0.1, \alpha \approx 10$, and $z_{c} \approx 0.2$. The source term is re-parameterized with the distinguished limit of $v_{1}=\sqrt{\delta} \nu_{1}$. This leads to a global breakdown in the region $z<z_{c}$, for which WKB approximations are commonly used. A WKB approximation will be developed in the next section, for now we continue using matched asymptotics and to ensure the self-consistency of the outer solution we choose $z_{c}=\sqrt{\delta} Z_{c}$ so that the change over region of the source term occurs in the inner region. Further, we take the distinguished limit $\alpha=\frac{\tilde{\alpha}}{\sqrt{\delta}}$ in the upper boundary condition. Under these 
parameterizations we get the following inner equation to leading order in $\sqrt{\delta}$,

$$
\begin{aligned}
& W_{\text {inner }}^{\prime \prime}(Z)-i W_{\text {inner }}(Z)=\left\{\begin{array}{ll}
-e^{-\frac{Z}{\nu_{1}}}, & Z \leq Z_{c} \\
-e^{-\frac{Z_{c}}{\nu_{1}}}, & Z>Z_{c}
\end{array},\right. \\
& \left.\frac{d W_{\text {inner }}}{d Z}\right|_{Z=0}=\tilde{\alpha} W_{\text {inner }} .
\end{aligned}
$$

This is to be solved in the regions $Z \leq Z_{c}$ and $Z>Z_{c}$. As before, this is exactly solvable with the same homogeneous solution as previously. We pick a particular solution of the form $W_{\text {inner,p }}=\tilde{c} e^{-\frac{Z}{\nu_{1}}}$ for $Z \leq Z_{c}$ and $W_{\text {inner,p }}=c$ for $Z>Z_{c}$ and solve for these constants.

$$
\begin{gathered}
\tilde{c}=-\frac{i+\frac{1}{\nu_{1}^{2}}}{\frac{1}{\nu_{1}^{4}}+1}, \\
c=-i e^{-\frac{Z_{c}}{\nu_{1}}} .
\end{gathered}
$$

The general solution of the inner solution is therefore given by

$$
W_{\text {inner }}= \begin{cases}\tilde{c}_{1} \exp (\sqrt{i} Z)+\tilde{c}_{2} \exp (-\sqrt{i} Z)-\left(\frac{i+\frac{1}{\nu_{1}^{2}}}{\frac{1}{\nu_{1}^{4}}+1}\right) e^{-\frac{Z}{\nu_{1}}} & , Z \leq Z_{c} \\ c_{1} \exp (\sqrt{i} Z)+c_{2} \exp (-\sqrt{i} Z)-i e^{-\frac{Z_{c}}{\nu_{1}}} & , Z>Z_{c}\end{cases}
$$

Matching with the outer solution we must set $c_{1}=0$. Since $g(Z)$ is continuous we ensure $W_{\text {inner }}$ and $W_{\text {inner }}^{\prime}$ are continuous at $Z=Z_{c}$ and satisfy the boundary condition at $Z=0$. Combining all three conditions the remaining constants are given by

$$
\begin{aligned}
& \tilde{c}_{1}=\frac{e^{-Z_{c}\left(\frac{1}{\nu_{1}}+\sqrt{i}\right)}\left(\alpha\left(\sqrt{i}-i \nu_{1}\right)-(\sqrt{i})^{3} \nu_{1}+i\right)}{2(\sqrt{i} \alpha+i)\left(\nu_{1}^{2}+i\right)} \\
& \tilde{c}_{2}=\frac{e^{-Z_{c}\left(\frac{1}{\nu_{1}}+\sqrt{i}\right)}\left(\alpha\left(i \nu_{1}-\sqrt{i}\right)-(\sqrt{i})^{3} \nu_{1}+i\right)+2(\sqrt{i})^{3} \nu_{1}\left(\alpha \nu_{1}+1\right)}{2(\sqrt{i} \alpha+i)\left(\nu_{1}^{2}+i\right)} \\
& c_{2}=\frac{1}{2(\sqrt{i} \alpha+i)\left(\nu_{1}^{2}+i\right)}\left(2(\sqrt{i})^{3} \nu_{1}\left(\alpha \nu_{1}+1\right)+e^{-Z_{c}\left(\frac{1}{\nu_{1}}+\sqrt{i}\right)}\right. \\
& \left.\quad \times\left(\alpha\left(i \nu_{1}-\sqrt{i}\right)-(\sqrt{i})^{3} \nu_{1}+i-e^{2 \sqrt{i} Z_{c}}\left(\alpha\left(\sqrt{i}+i \nu_{1}\right)+(\sqrt{i})^{3} \nu_{1}+i\right)\right)\right)
\end{aligned}
$$

\subsubsection{Interpretations and Numerical Comparisons}

Equation 4.8, the inner solution, is seen to have the form of a stationary wave (as with the outer solution), being the particular solution, and a travelling wave 
component from the homogeneous solution. The decay of the stationary wave is the same as the distributed source term, whereas the decay of the travelling wave is given by the diffusivity at the surface, $\delta$. Travelling waves will only be visible in the solution plots if the decay of the travelling waves is significantly slower than the decay of the stationary waves, that is $\frac{1}{\sqrt{2 \delta}} \ll \frac{1}{v_{1}}$ is required for travelling waves to be present.

We compare numerical solutions of equation 3.13 to the outer solution (equation 4.6) and both inner solutions (equations 4.7 and 4.8 ) for a variety of parameter values in Figures 4.1 and 4.2 . We see that for extremely small values of $\delta$ both inner solutions converge to the exact solution in their range of validity, and the exact solution converges to the outer solution in larger subsets of the interval $[0,1]$. It is seen that the revised inner solution (equation 4.8) performs significantly better than equation 4.7 for physically relevant values, although it does not capture the change of wave region - from travelling to stationary. The solid-state greenhouse effect may be studied using the revised inner solution as it is seen to always occur within its region of validity.

We do not focus on the inner region near $z=1$ as it is of little physical interest and has a similar mathematical form to the boundary layer near $z=0$. Matched asymptotics of equation 3.18 yield similar results, with a trivial outer solution $W_{\text {outer }}=0$ and the solution to a constant parameter heat equation in the inner solution.

\subsection{WKB Approximation}

The inner and outer solutions (equations 4.6 and 4.8) recreate the numerical solution well in their respective regions of validity, however, do not perform well in the intermediate region where the travelling and stationary waves meet for physically relevant parameter values. Further, the inner solution is simply the solution to the constant parameter heat equation and, although performing well numerically, does not give insight into the non-constant parameter case. In this section we derive a uniform approximation to the solutions of equations 3.13 and 3.18 . We begin by analysing the homogeneous equation and use the Green's function method [9] to obtain approximate solutions to the non-homogeneous equation. 

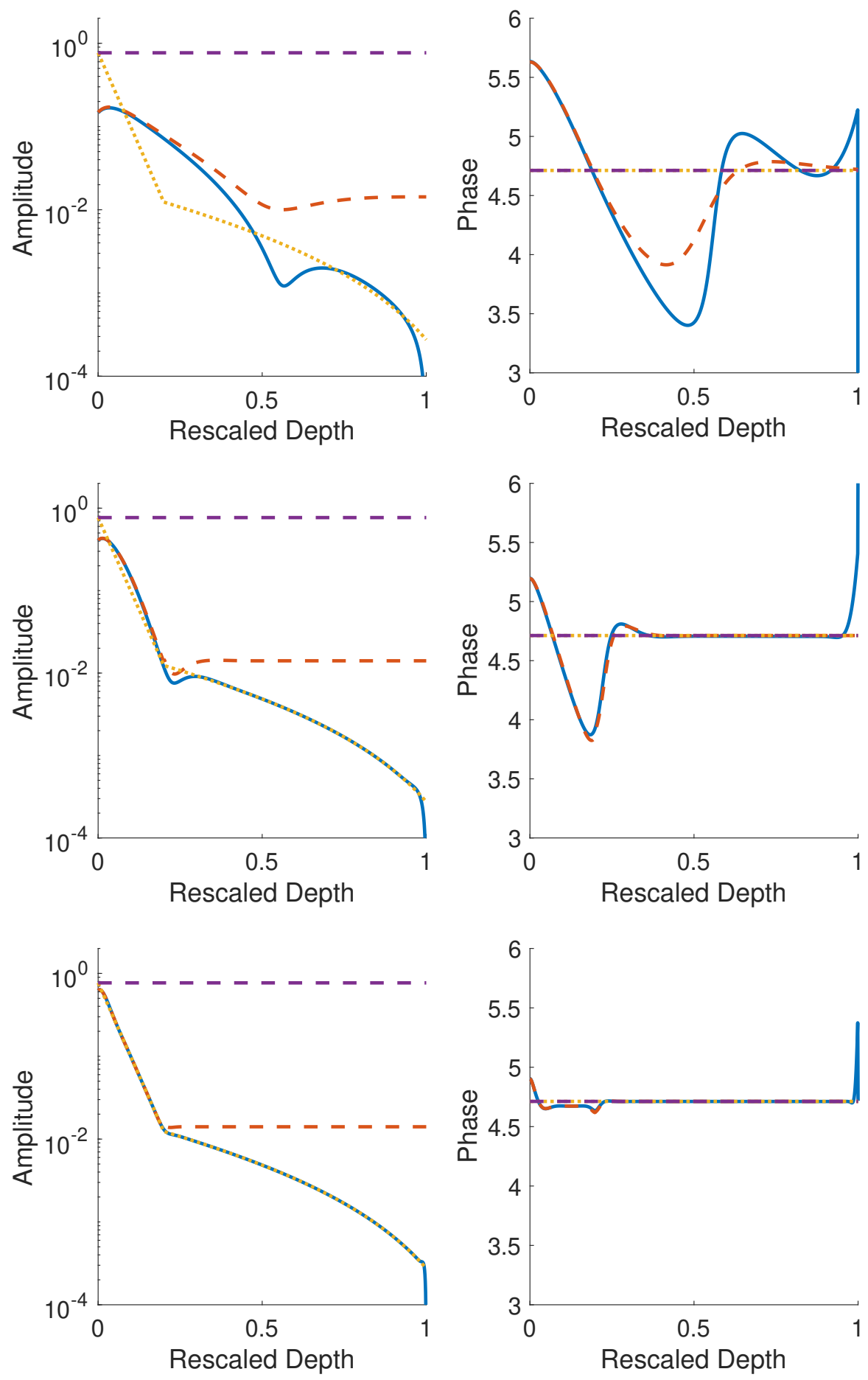

Figure 4.1: The two derivations of the inner solution, equation 4.7 (purple dashed), equation 4.8 (orange dashed), and the outer solution, equation 4.6 (yellow dotted), compared with numerical solutions of equation 3.13 (blue solid) for parameter values as follow: $\alpha=10, v_{1}=0.05, v_{2}=0.5, z_{c}=0.2, \delta=10^{-2}$ (top) $10^{-3}$ (middle) $10^{-4}$ (bottom). 

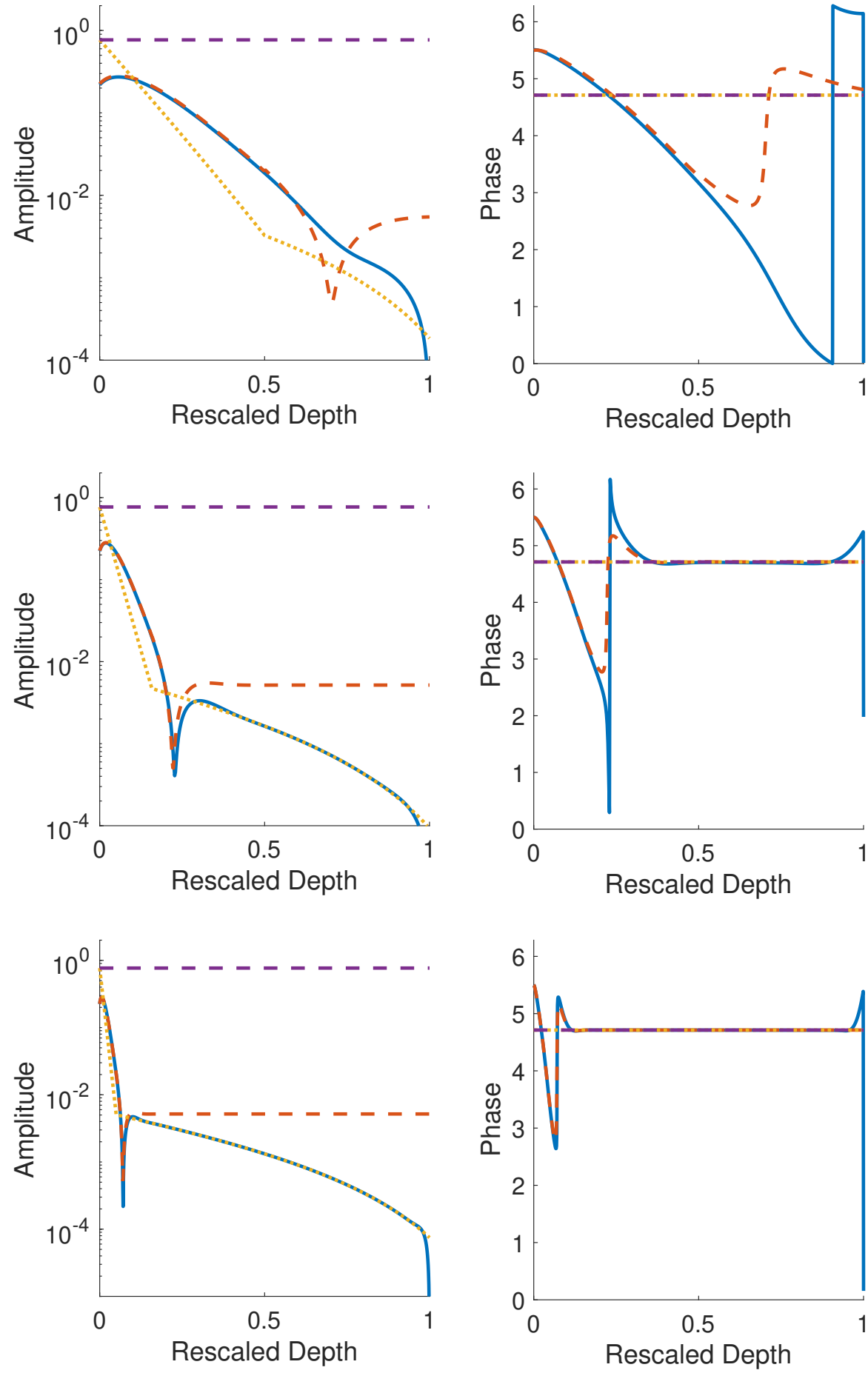

Figure 4.2: The two derivations of the inner solution, equation 4.7 (purple dashed), equation 4.8 (orange dashed), and the outer solution, equation 4.6 (yellow dotted), compared with numerical solutions of equation 3.13 (blue solid) for parameter values as follows: $\alpha=1 / \sqrt{\delta}, v_{1}=\sqrt{\delta}, v_{2}=0.5, z_{c}=5 \times \sqrt{\delta}, \delta=10^{-2}$ (top) $10^{-3}$ (middle) $10^{-4}$ (bottom). 


\subsubsection{Homogeneous System}

The homogeneous system (equation 3.18) is as follows:

$$
\delta W^{\prime \prime}-\frac{i}{f(z)} W=0 .
$$

Homogeneous second order differential equations of this form have been studied thoroughly, and hence we can immediately write down the leading order WKB approximation as given in [9] and described previously.

$$
W \sim W_{\mathrm{h}, \mathrm{WKB}}=\exp \left(\frac{1}{\epsilon} S_{0}(z)+S_{1}(z)+\mathcal{O}(\epsilon)\right)
$$

with

$$
\begin{aligned}
& \epsilon^{2}=\delta \\
& S_{0}(z)= \pm \int_{0}^{z} \sqrt{\frac{i}{f(s)}} d s \\
& S_{1}(z)=-\frac{1}{4} \ln \left(\frac{i}{f(z)}\right) .
\end{aligned}
$$

The \pm gives rise to two solutions which can be linearly combined to form a solution of the form

$$
W_{\mathrm{h}, \mathrm{WKB}}=h_{1} f^{\frac{1}{4}}(z) \exp \left[\frac{1}{\sqrt{\delta}} \int_{0}^{z} \sqrt{\frac{i}{f(t)}} d t\right]+h_{2} f^{\frac{1}{4}}(z) \exp \left[-\frac{1}{\sqrt{\delta}} \int_{0}^{z} \sqrt{\frac{i}{f(t)}} d t\right]
$$

where $h_{1}$ and $h_{2}$ are constants to be determined via the boundary conditions. This, together with the time oscillation, has the form of travelling thermal wave as can be seen in the similarity to equation 4.8 with assumed constant $f(z)$. From here on we will drop the subscript WKB, with a subscript of $h$ representing approximate solutions to the homogeneous system.

\section{Validity of the Leading Order WKB Approximation}

To test the validity of this leading order approximation we compute the next term in the WKB series, which must be much less than 1 and also much less than the previous terms.

$$
\sqrt{\delta} S_{2}(z)=\sqrt{\delta} \int_{0}^{z} \frac{1}{32}\left(\frac{i}{\mathrm{f}(t)}\right)^{3 / 2}\left(4 \mathrm{f}(t) \mathbf{f}^{\prime \prime}(t)-3 \mathbf{f}^{\prime}(t)^{2}\right) d t
$$


For our parameterization, $f(z)$ doesn't have a zero on $[0,1]$ and in fact $f(z)$ is bounded between 1 and 0.1 for all salinity profiles, $f^{\prime}(z)$ is between 0 and -1.5 and $f^{\prime \prime}(z) \approx-1$. The next term in the WKB series is bounded by the following.

$$
\begin{aligned}
\left|\sqrt{\delta} S_{2}(z)\right| & <\left|\sqrt{\delta} \int_{0}^{z} \frac{1}{32}\left(\frac{i}{0.1}\right)^{3 / 2}\left(4 \times 1(-1)-3(-1.5)^{2}\right) d t\right| \\
& \approx \sqrt{\delta} \frac{1}{3} 10^{3 / 2} z \approx 10 \sqrt{\delta} z
\end{aligned}
$$

This term gives the error estimation of the WKB approximation with the first two terms, which is valid on $0 \leq z \leq 1$ if $\delta<10^{-2}$. As seen in our parameterization of $\delta$ for physically relevant values, this is the case! For a value of $\delta=10^{-3}$ we can expect a maximum error of about $\sqrt{10^{-3}} \times 10 \approx 0.3$ or $30 \%$ over $0 \leq z \leq 1$. This is an over-estimation for the error near the surface as it is highly sensitive to the changes in $f(z)$, with the large error estimation due to the small value of $f(z)$ near $z=1$. On the interval $0 \leq z \leq 0.8, f(z)$ is bounded between 1 and 0.3 , hence the term $\mid \sqrt{\delta} S_{2}(z)$ is bounded by $2 \sqrt{\delta}$, giving a maximum error of $6 \%$ for $\delta=10^{-3}$.

\section{Oscillating Air Temperatures - Boundary Conditions}

Here we will show that the dominant balance of equal thermal wave and air temperature wave amplitudes is the only possible balance to get non-trivial solutions. If we had selected a dominant balance where the fast time dependence of the air temperatures was a different order other than $\epsilon$, matching the powers of $\epsilon$ in the boundary conditions would not include the non-homogeneity in the boundary conditions of equation 3.18 . This would result in the following boundary conditions for $W_{\mathrm{h}}$.

$$
\begin{aligned}
& W_{\mathrm{h}}(1)=0, \\
& W_{\mathrm{h}}^{\prime}(0)=\alpha W(0) .
\end{aligned}
$$

From equation 4.12 , the boundary conditions are written explicitly as

$$
\begin{aligned}
& h_{1} \exp \left[\frac{2}{\sqrt{\delta}} \int_{0}^{1} \sqrt{\frac{i}{f(t)}} d t\right]+h_{2}=0, \\
& h_{1}\left[\frac{1}{4} f^{\prime}(0)+\frac{\sqrt{i}}{\sqrt{\delta}}-\alpha\right]+h_{2}\left[\frac{1}{4} f^{\prime}(0)-\frac{\sqrt{i}}{\sqrt{\delta}}-\alpha\right]=0 .
\end{aligned}
$$

In general, the only solution to these boundary conditions is the trivial solution except for specific parameter values of $\delta, \alpha$ for which the matrix of coefficients 
is non-invertible. This is physically interpreted as no driving oscillatory 'force' (being a source term or boundary condition) being present and hence no thermal waves are present.

We turn to the dominant balance stated earlier and apply the following boundary conditions on the leading order WKB approximation for equation 3.18

$$
\begin{aligned}
& W_{\mathrm{h}}(1)=0, \\
& W_{\mathrm{h}}^{\prime}(0)=\alpha(W(0)-A) .
\end{aligned}
$$

The boundary conditions will determine the constants $h_{1}, h_{2}$. Using $\gamma^{\prime}(0)=\sqrt{\frac{i}{\delta}}$ and the form of $W_{\mathrm{h}}$ we simplify the boundary conditions to

$$
\begin{aligned}
& h_{1} e^{2 \gamma(1)}+h_{2}=0 \\
& h_{1}\left[\frac{1}{4} f^{\prime}(0)+\sqrt{\frac{i}{\delta}}-\alpha\right]+h_{2}\left[\frac{1}{4} f^{\prime}(0)-\sqrt{\frac{i}{\delta}}-\alpha\right]=-A \alpha .
\end{aligned}
$$

Solving the boundary conditions simultaneously, we get the following solutions for the constants $h_{1}, h_{2}$.

$$
\begin{aligned}
& h_{1}=-\frac{2 A \alpha e^{-\gamma(1)}}{\sinh (\gamma(1))\left(4 \alpha-f^{\prime}(0)\right)+4 \sqrt{\frac{i}{\delta}} \cosh (\gamma(1))} . \\
& h_{2}=\frac{2 A \alpha e^{\gamma(1)}}{\sinh (\gamma(1))\left(4 \alpha-f^{\prime}(0)\right)+4 \sqrt{\frac{i}{\delta}} \cosh (\gamma(1))} .
\end{aligned}
$$

Since $\gamma(1) \sim \frac{1}{\sqrt{\delta}}, \delta \rightarrow 0$, the hyperbolic trig functions are well approximated with exponentials to give the following approximate constants.

$$
\begin{gathered}
h_{1} \approx-\frac{4 A \alpha e^{-2 \gamma(1)}}{4 \alpha-f^{\prime}(0)+4 \sqrt{\frac{i}{\delta}}} . \\
h_{2} \approx \frac{4 A \alpha}{4 \alpha-f^{\prime}(0)+4 \sqrt{\frac{i}{\delta}}} .
\end{gathered}
$$

The growing exponential term $h_{1} f^{\frac{1}{4}}(z) e^{\gamma(z)}$ is only non-negligible near $z=1$, where it ensures the boundary condition at $z=1$ is met. This term is the reflected thermal wave from the bottom boundary. Hence the dominant behaviour of the solution is a travelling wave decaying into the ice from the upper surface. The parameter $\alpha$ dictates the atmosphere-ice thermal contact. For $\alpha=0$ we get $h_{2}=$ $h_{1}=0$, the ice is completely isolated from the atmosphere and hence no thermal waves are present. For $\alpha \rightarrow \infty$ we get $h_{2} \rightarrow A$, the ice is in perfect thermal contact 
with the atmosphere. We have assumed, as is physically relevant, that $f(z)$ varies more slowly than $\gamma(z)$, so $h_{1}<A$ for all parameter values.

We see excellent numerical agreement between the leading order WKB approximation above and numerical solutions to equation 3.18. Examples of such comparisons are provided in Figure 4.3 for a diurnal thermal wave of amplitude $2{ }^{\circ} \mathrm{C}$.

\subsubsection{Non-Homogeneous Case - Distributed Source}

We approach approximating the solution to the non-homogeneous system (equation 3.13) using the Green's function method and the WKB approximation, inspired by the demonstration in [9]. We will construct an approximate Green's function using the $\mathrm{WKB}$ method and use it to get approximate solutions to equation 3.13 . We write the approximate solution as

$$
\begin{array}{r}
W_{\text {WKB }}(z)=\int G(z, s)\left(-\frac{g(s)}{f(s)}\right) d s \\
\delta^{\prime} \frac{d^{2}}{d z^{2}} G(z, s)-\frac{i}{f(z)} G(z, s)=\delta(z-s)
\end{array}
$$

where the constant $\delta$ has been relabeled $\delta^{\prime}$ to distinguish the delta function in the above equation, but from now on we drop the'. Again we drop the subscript WKB from here on.

For all values of $z \neq s$, the equation reduces to the homogeneous case (equation 3.18) that was approximated previously. Hence we get two approximate solutions, one in the region $z>s$ and one in the region $z<s$ both taking the form of equation 4.12 .

$$
G(z, s) \sim \begin{cases}c_{1}(s) f^{\frac{1}{4}}(z) \exp [\gamma(z)]+\tilde{c}_{1}(s) f^{\frac{1}{4}}(z) \exp [-\gamma(z)], & z<s \\ c_{2}(s) f^{\frac{1}{4}}(z) \exp [\gamma(z)]+\tilde{c}_{2}(s) f^{\frac{1}{4}}(z) \exp [-\gamma(z)], & z>s\end{cases}
$$

with

$$
\gamma(z)=\sqrt{\frac{i}{\delta}} \int_{0}^{z} \sqrt{\frac{1}{f(t)}} d t
$$

The constants $c_{1}, c_{2}$ etc. are to be found from the boundary conditions and the patching conditions. The approximate solution for $W(z)$ is written as

$$
W(z) \sim \int_{0}^{z} G_{2}(z, s)\left(-\frac{g(s)}{f(s)}\right) d s+\int_{z}^{1} G_{1}(z, s)\left(-\frac{g(s)}{f(s)}\right) d s
$$



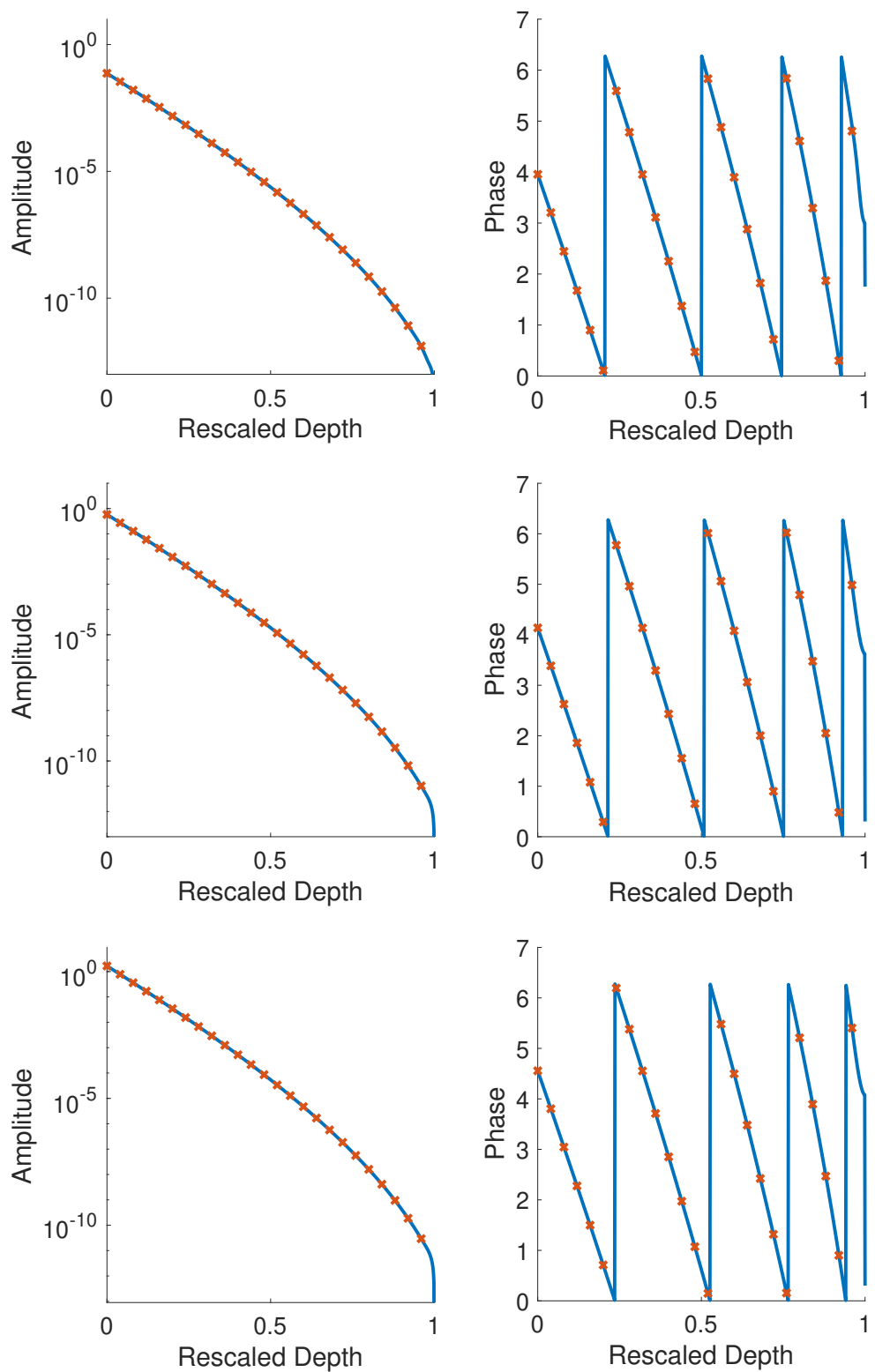

Figure 4.3: Comparison between numerical solutions of equation 3.18 (solid blue) and the leading order WKB approximation (equation 4.12) with $h_{1}=0$ and $h_{2}$ as in equation 4.14 (orange $x^{\prime} \mathrm{s}$ ). A surface temperature of $-5^{\circ} \mathrm{C}$ and an ice depth of $H=2 \mathrm{~m}$ were used, along with $\alpha=1$ (top), 10 (middle) and 100 (bottom). 
where the subscript corresponds to the subscript of the constants in each Greens function component.

The patching conditions on the Greens function states that $G(z, s)$ is continuous at $z=s$ and $\frac{d}{d z} G(z, s)$ has a discontinuity of $\frac{1}{\delta}$ at $z=s$. We get the following two conditions

$$
\begin{aligned}
& c_{1}(s) \exp [2 \gamma(s)]+\tilde{c}_{1}(s)-c_{2}(s) \exp [2 \gamma(s)]-\tilde{c}_{2}(s)=0, \\
& c_{1}(s) \frac{1}{4} f^{-\frac{3}{4}}(s) f^{\prime}(s) \exp [\gamma(s)]+c_{1}(s) f^{\frac{1}{4}}(s) \gamma^{\prime}(s) \exp [\gamma(s)] \\
& +\tilde{c}_{1}(s) \frac{1}{4} f^{-\frac{3}{4}}(s) f^{\prime}(s) \exp [-\gamma(s)]-\tilde{c}_{1}(s) f^{\frac{1}{4}}(s) \gamma^{\prime}(s) \exp [-\gamma(s)] \\
& -c_{2}(s) \frac{1}{4} f^{-\frac{3}{4}}(s) f^{\prime}(s) \exp [\gamma(s)]-c_{2}(s) f^{\frac{1}{4}}(s) \gamma^{\prime}(s) \exp [\gamma(s)] \\
& -\tilde{c}_{2}(s) \frac{1}{4} f^{-\frac{3}{4}}(s) f^{\prime}(s) \exp [-\gamma(s)]+\tilde{c}_{2}(s) f^{\frac{1}{4}}(s) \gamma^{\prime}(s) \exp [-\gamma(s)]=-\frac{1}{\delta} .
\end{aligned}
$$

Two more boundary conditions are needed to fix the values of $c_{1}, c_{2}, \tilde{c}_{1}, \tilde{c}_{2}$, these are the boundary conditions imposed on $W(z)$ treated next.

\section{Boundary Conditions}

For a generalised homogeneous boundary condition on $W(z)$, we apply the same boundary condition to the Green's function to retrieve a relevant solution [9].

$$
\begin{aligned}
& a_{1} W\left(z_{1}\right)+a_{2} W^{\prime}\left(z_{1}\right)=0, \\
& a_{1} G\left(z_{1}, s\right)+a_{2} G^{\prime}\left(z_{1}, s\right)=0,
\end{aligned}
$$

with primes denoting differentiation with respect to $z$. The boundary conditions for $W(z)$ are

$$
W^{\prime}(0)=\alpha W(0), \quad W(1)=0 .
$$

To fulfill the first boundary condition we require

$$
G^{\prime}(0, s)=\alpha G(0, s)
$$

where the derivative $G^{\prime}(0, s)$ is to be interpreted as $\lim _{z \rightarrow 0^{+}} \frac{\partial G(z, s)}{\partial z}$ as $W(z)$ is given in terms of an integral of $G$ with respect to $s$. Substituting and simplifying with $f(0)=1, \gamma(0)=0$ and $\gamma^{\prime}(0)=\sqrt{\frac{i}{\delta}}$, we get

$$
c_{1}(s)\left(\frac{1}{4} f^{\prime}(0)+\sqrt{\frac{i}{\delta}}-\alpha\right)+\tilde{c}_{1}(s)\left(\frac{1}{4} f^{\prime}(0)-\sqrt{\frac{i}{\delta}}-\alpha\right)=0 .
$$


For the second boundary condition, we require

$$
\exp [2 \gamma(1)] c_{2}(s)+\tilde{c}_{2}(s)=0 \text {. }
$$

Combining all four boundary and patching conditions we solve explicitly for the coefficients $c_{1}, c_{2}, \tilde{c}_{1}, \tilde{c}_{2}$ as

$$
\begin{aligned}
& c_{1}(s)=\frac{\sinh (\gamma(1)-\gamma(s))}{\sqrt[4]{\mathbf{f}(s)} \gamma^{\prime}(s)} \frac{\left(-4 \alpha-4 \sqrt{\frac{i}{\delta}}+\mathbf{f}^{\prime}(0)\right)}{2 \delta\left(\sinh (\gamma(1))\left(4 \alpha-\mathbf{f}^{\prime}(0)\right)+4 \sqrt{\frac{i}{\delta}} \cosh (\gamma(1))\right)}, \\
& \tilde{c}_{1}(s)=-\frac{\sinh (\gamma(1)-\gamma(s))}{\sqrt[4]{\mathbf{f}(s)} \gamma^{\prime}(s)} \frac{\left(-4 \alpha+4 \sqrt{\frac{i}{\delta}}+\mathbf{f}^{\prime}(0)\right)}{2 \delta\left(\sinh (\gamma(1))\left(4 \alpha-\mathbf{f}^{\prime}(0)\right)+4 \sqrt{\frac{i}{\delta}} \cosh (\gamma(1))\right)}, \\
& c_{2}(s)=\frac{e^{-\gamma(1)}\left(\left(4 \alpha-\mathbf{f}^{\prime}(0)\right) \sinh (\gamma(s))+4 \sqrt{\frac{i}{\delta}} \cosh (\gamma(s))\right)}{2 \delta \sqrt[4]{\mathbf{f}(s)} \gamma^{\prime}(s)\left(\sinh (\gamma(1))\left(4 \alpha-\mathbf{f}^{\prime}(0)\right)+4 \sqrt{\frac{i}{\delta}} \cosh (\gamma(1))\right)}, \\
& \tilde{c}_{2}(s)=\frac{e^{\gamma(1)}\left(\left(-4 \alpha+\mathbf{f}^{\prime}(0)\right) \sinh (\gamma(s))-4 \sqrt{\frac{i}{\delta}} \cosh (\gamma(s))\right)}{2 \delta \sqrt[4]{\mathbf{f}(s)} \gamma^{\prime}(s)\left(\sinh (\gamma(1))\left(4 \alpha-\mathrm{f}^{\prime}(0)\right)+4 \sqrt{\frac{i}{\delta}} \cosh (\gamma(1))\right)} .
\end{aligned}
$$

\section{Numerical Comparison}

Numerical approximations are calculated using the above constants and equation 4.17 using MATLAB's builtin integral function. These appear to recreate the numerical solutions of equation 3.13 extraordinarily well for a wide range of values, as presented in Figures 4.4 and 4.5 . We see that as expected by the error estimations, the largest relative error occurs near $z=1$.

The leading order behaviour is written in terms of complicated integrals in equation 4.17. In the next section we apply relevant approximations to the above leading order behaviour in search of analytical approximations to it. 

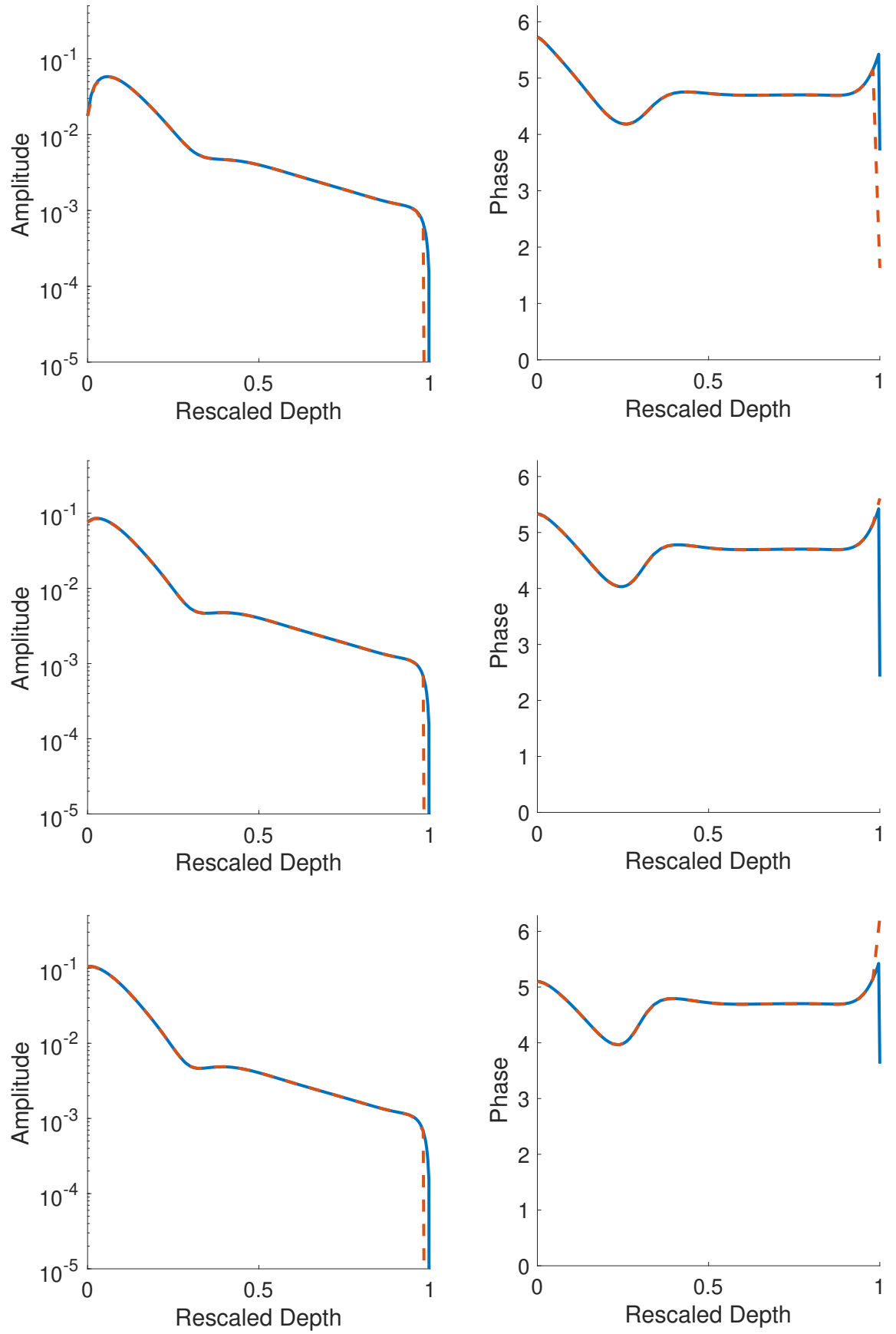

Figure 4.4: Numerically calculated WKB approximations, equation 4.17, (dashed red) compared with numerical solutions (solid blue) of equation 3.13. The parameters used were: $v_{1}=0.07, v_{2}=0.25, z_{c}=0.2, \delta=3 \times 10^{-3}$ and $\alpha=100$ (top), 10 (middle) and 1 (bottom). 

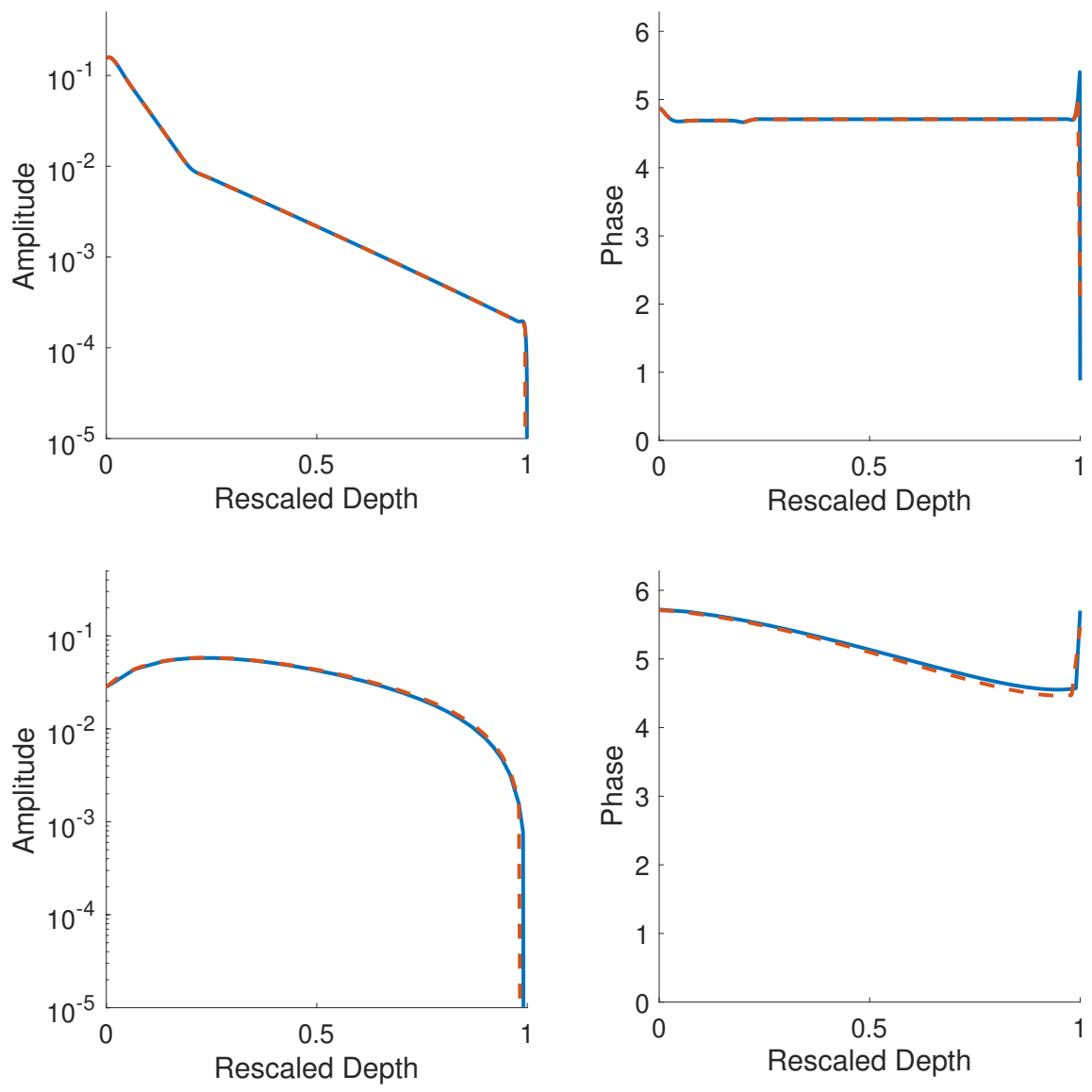

Figure 4.5: Numerically calculated WKB approximations, equation 4.17. (dashed red) compared with numerical solutions (solid blue) of equation 3.13 The parameters used were: $\alpha=10, v_{1}=0.07, v_{2}=0.25, z_{c}=0.2, \delta=10^{-4}$ (top) and $10^{-1}$ (bottom). The $W K B$ approximation recreates the numerical solution fairly accurately over the entire range even for relatively high (and un-physical) values of $\delta$. 


\subsubsection{Asymptotic form in the limit $\delta \rightarrow 0$}

For all relevant salinity profiles and surface temperatures, $\gamma(z)$ is an increasing function on $z \in[0,1]$, and $\gamma(z) \sim \frac{1}{\sqrt{\delta}}$. In the limit $\delta \rightarrow 0, \gamma(1) \rightarrow \infty$ and so $\sinh (\gamma(1)) \approx \cosh (\gamma(1)) \approx \frac{1}{2} e^{\gamma(1)}$. This is valid for all values of $z$ and $s$.

$$
\begin{aligned}
& c_{1}(s) \approx-\frac{\sinh (\gamma(1)-\gamma(s))}{\sqrt[4]{\mathrm{f}(s)} \gamma^{\prime}(s)} \frac{1}{\delta e^{\gamma(1)}}, \\
& \tilde{c}_{1}(s) \approx-\frac{\sinh (\gamma(1)-\gamma(s))}{\sqrt[4]{\mathrm{f}(s)} \gamma^{\prime}(s)} \frac{\beta}{\delta e^{\gamma(1)}}, \\
& c_{2}(s) \approx \frac{e^{-2 \gamma(1)}}{2 \delta \sqrt[4]{\mathbf{f}(s)} \gamma^{\prime}(s)}\left[e^{\gamma(s)}+e^{-\gamma(s)} \beta\right], \\
& \tilde{c}_{2}(s) \approx \frac{-1}{2 \delta \sqrt[4]{\mathrm{f}(s)} \gamma^{\prime}(s)}\left[e^{\gamma(s)}+e^{-\gamma(s)} \beta\right],
\end{aligned}
$$

with

$$
\beta=\frac{-4 \alpha+f^{\prime}(0)+4 \sqrt{\frac{i}{\delta}}}{4 \alpha-f^{\prime}(0)+4 \sqrt{\frac{i}{\delta}}} .
$$

The approximate form of the Green's function (separated into the two regions $z>s$ and $z<s$ ) from equation 4.15 is as below,

$$
\begin{aligned}
& G_{1}(z, s) \approx \frac{\sinh (\gamma(s)-\gamma(1))}{\gamma^{\prime}(s)} \frac{\sqrt[4]{\mathfrak{f}(z)}}{\sqrt[4]{\mathfrak{f}(s)} \delta e^{\gamma(1)}}(\exp (\gamma(z))+\beta \exp (-\gamma(z))) \\
& G_{2}(z, s) \approx \frac{\sqrt[4]{\mathfrak{f}(z)}}{2 \delta \sqrt[4]{\mathfrak{f}(s)} \gamma^{\prime}(s)}\left[e^{\gamma(s)}+\beta e^{-\gamma(s)}\right]\left(e^{-2 \gamma(1)} \exp (\gamma(z))-\exp (-\gamma(z))\right) .
\end{aligned}
$$

These Green's functions may further be approximated by disregarding terms with $e^{-2 \gamma(1)}$. As was noted for the homogeneous system, these are associated with the boundary condition at $z=1$ and reflected thermal waves. Terms of this form only become significant relative to the other terms near $s=1$. At these points the contribution of the Green's function to $W(z)$ is subdominant to the contribution in the remainder of the region due to the exponentially decreasing nature of the source term $\frac{g(s)}{f(s)}$ and the Green's function being of order $e^{-\gamma(1)}$. We further approximate the Green's function as below.

$$
\begin{aligned}
& G_{1}(z, s) \approx \frac{e^{-\gamma(s)}}{2 \gamma^{\prime}(s)} \frac{\sqrt[4]{\mathbf{f}(z)}}{\sqrt[4]{\mathbf{f}(s)} \delta}(\exp (\gamma(z))+\beta \exp (-\gamma(z))) \\
& G_{2}(z, s) \approx-\frac{\sqrt[4]{\mathbf{f}(z)}}{2 \delta \sqrt[4]{\mathbf{f}(s)} \gamma^{\prime}(s)}\left[e^{\gamma(s)}+\beta e^{-\gamma(s)}\right] \exp (-\gamma(z))
\end{aligned}
$$


What does this mean for the form of $W(z)$ ? We separate the approximate solutions into $W(z)=W_{1}(z)+W_{2}(z)$, each corresponding to the Green's function integral with the same subscript. Using $\gamma^{\prime}(s)=\sqrt{\frac{i}{\delta f(s)}}$ we simplify the expressions as below.

$$
\begin{aligned}
W_{1}(z) & \sim \int_{z}^{1} G_{1}(s, z)\left(-\frac{g(s)}{f(s)}\right) d s \\
& \approx-\sqrt[4]{\mathrm{f}(z)}(\exp (\gamma(z))+\beta \exp (-\gamma(z))) \frac{1}{2 \sqrt{i \delta}} \int_{z}^{1} e^{-\gamma(s)} f^{-\frac{3}{4}}(s) g(s) d s, \\
W_{2}(z) & \sim \int_{0}^{z} G_{2}(s, z)\left(-\frac{g(s)}{f(s)}\right) d s \\
& \approx \sqrt[4]{\mathrm{f}(z)} \exp (-\gamma(z)) \frac{1}{2 \sqrt{i \delta}} \int_{0}^{z}\left[e^{\gamma(s)}+\beta e^{-\gamma(s)}\right] f^{-\frac{3}{4}}(s) g(s) d s
\end{aligned}
$$

It is worthy to note that we have not assumed any region of validity for the above approximations, they are valid and introduce only small errors over the entire range $z \in[0,1]$ in the limit $\delta \rightarrow 0$. The approximate solution has now been written in terms of the following integrals.

$$
\begin{aligned}
& \tilde{I}_{1}(z)=\int_{z}^{1} e^{-\gamma(s)} f^{-\frac{3}{4}}(s) g(s) d s, \\
& I_{2}(z)=\int_{0}^{z} e^{\gamma(s)} f^{-\frac{3}{4}}(s) g(s) d s, \\
& \tilde{I}_{2}(z)=\int_{0}^{z} e^{-\gamma(s)} f^{-\frac{3}{4}}(s) g(s) d s .
\end{aligned}
$$

With the relevant forms of the parameter functions $f(z), g(z)$ these integrals cannot be written in terms of elementary functions. However, the leading order behaviour of these integrals can be evaluated by using methods as described in [9] and as introduced in section 4.1. We re-write

$$
\gamma(s)=x \phi(s), \quad x=\frac{1}{\sqrt{\delta}}, \quad \phi(s)=\sqrt{i} \int_{0}^{s} f^{-\frac{1}{2}}(t) d t
$$

to highlight the large parameter present in the exponent of the integrands of the above integrals. Next we evaluate the leading order contribution of these integrals in the limit $\delta \rightarrow 0$ corresponding to $x \rightarrow \infty$.

\section{Method of Steepest Descent}

We will need to evaluate four integrals in general in order to approximate the integrals of interest (equations 4.244 .26 ). These are as follows 


$$
\begin{aligned}
& J_{1}=\int_{a}^{b} e^{-\frac{1}{\sqrt{\delta}} \phi(s)} f^{-\frac{3}{4}}(s) g(s) d s, \\
& J_{2}=\int_{a}^{b} e^{\frac{1}{\sqrt{\delta}} \phi(s)} f^{-\frac{3}{4}}(s) g(s) d s, \\
& J_{3}=\int_{a}^{b} e^{-\frac{1}{\sqrt{\delta}}\left(\phi(s)+\frac{s}{\nu_{1}}\right)} f^{-\frac{3}{4}}(s) \tilde{g}(s) d s, \\
& J_{4}=\int_{a}^{b} e^{\frac{1}{\sqrt{\delta}}\left(\phi(s)-\frac{s}{\nu_{1}}\right)} f^{-\frac{3}{4}}(s) \tilde{g}(s) d s .
\end{aligned}
$$

$J_{1}$ and $J_{2}$ give the contribution of integrals where $g^{\prime}(s)$ is negligible compared to the decay of the exponential with the large parameter (in the region $s>z_{c}$ ), whereas $J_{3}$ and $J_{4}$ give contributions in the region where $g^{\prime}(s)$ is comparable to the decay of the exponential with the large parameter (in the region $s<z_{c}$ ). We have written, as before,

$$
\gamma(s)=\sqrt{\frac{i}{\delta}} \int_{0}^{s} f^{-\frac{1}{2}}(t) d t=\frac{1}{\sqrt{\delta}} \phi(s),
$$

to make use of the small parameter, and $g(s)=e^{-\frac{s}{\sqrt{\delta} \nu_{1}}} \tilde{g}(s)$ where $\tilde{g}(s)$ is the slowly varying component of $g(s)$. In order to apply the method of steepest descent, we need some knowledge of the contours of constant imaginary (and constant real) components. Using a quadratic approximation to $f(s), f(s)=1-q_{1} z-q_{2} z^{2}$, as discussed in Figure $3.10, \phi(s)$ takes on the form

$$
\phi_{q}(s)=\frac{i \log \left(2 \sqrt{f(s)}-\frac{i f^{\prime}(s)}{\sqrt{q_{2}}}\right)}{\sqrt{q_{2}}}-\frac{i \log \left(2-\frac{i q_{1}}{\sqrt{q_{2}}}\right)}{\sqrt{q_{2}}} .
$$

This is multi-valued as $f(s)$ has two zeros. From here on we will use the quadratic fitting to $f(z)$ and will denote $\phi(s)=\phi_{q}(s)$. The real and imaginary components of $\phi(s)$ are shown in Figure 4.6. The function $\phi$ can also be written as a complex valued arcsin. These expressions do not yield any obvious method of determining the contours of constant imaginary and real parts. However, we can extract some of this information as will be shown in a few paragraphs.

The branch cuts present in Figure 4.6 connect the zeroes of $f$ to infinity, with the default branch cuts of Mathematica shown. However, the branch cuts can be chosen to be any connected curve connecting the branch points (the zeroes of $f$ and infinity). For all salinity profiles $f(s)$ does not have a zero on the interval $[0,1]$. The contours of integration $(z, 1)$ or $(0, z)$ in equations $4.27,4.28,4.29$ and 4.30 can be deformed into two contours of constant phase, $C_{1}$ and $C_{3}$, from each 

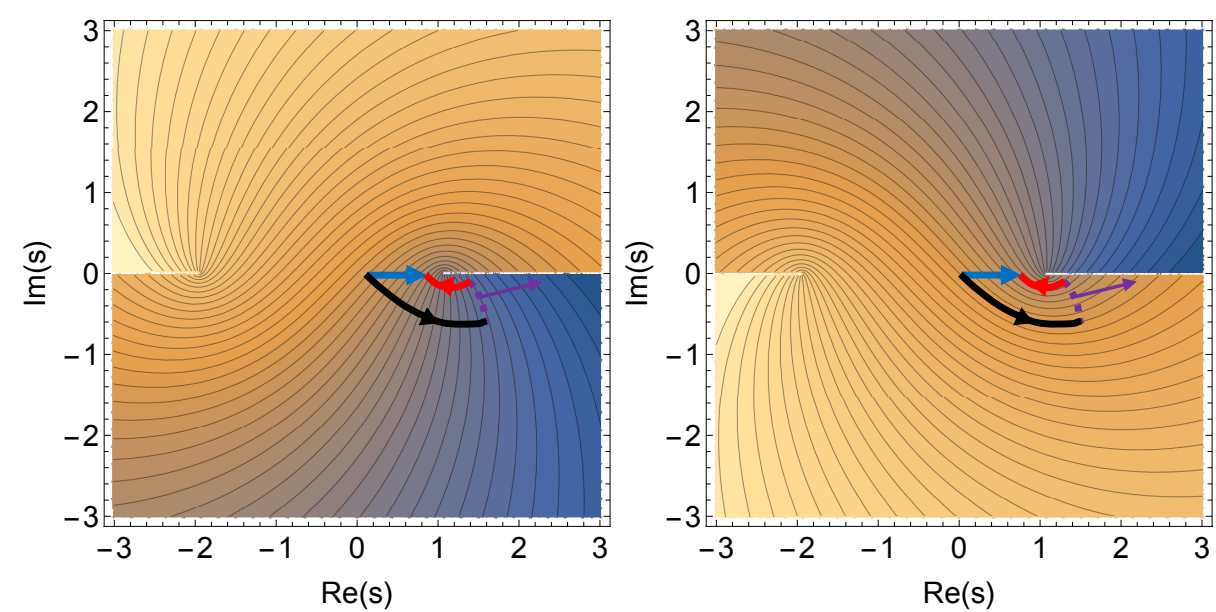

Figure 4.6: The real (left) and imaginary (right) components of $+\phi(s)$ in the complex plane. Note the two branch cuts connecting the zeros of $f(s)$ to infinity. A typical contour of integration is indicated with a blue arrow. Using the method of steepest descent, this contour is deformed to a contour of integration indicated by the black, purple and red lines (referred to in the text via $C_{1}, C_{2}$ and $C_{3}$, respectively).

end point to a point at infinity. These are connected with a contour $C_{2}$ being a contour of constant real component at infinity. We select the branch cuts for $\phi(s)$ appropriately, for example, a contour of constant imaginary component of $\phi(s)$ not between $C_{1}$ and $C_{3}$, to ensure the contours of integration do not cross branch cuts. Further, since $C_{1}$ and $C_{3}$ are contours of constant imaginary component, they are contours of steepest descent/ascent for the real component of $\phi(s)$ [9]. That means that the real component of $\phi(s)$ along the contour $C_{2}$ is bounded by $\max (\phi(a), \phi(b))$.

We assume we have the three curves $C_{1,2,3}$ all parameterized with constant speed, $\left|c_{n}^{\prime}(t)\right|=1$ for all $t \in\left[0, l_{n}\right]$ with $l_{n}$ being the length of each contour. For an appropriate branch cut choice the contours $C_{1,3}$ tend to $\infty$ where the real component of $\phi(s)$ tends to $-\infty$, as the contours $C_{1,3}$ are of steepest descent/ascent. It is worthy to note that we have not needed to assume that $\delta \rightarrow 0$ yet, the negligible contribution from the integral along $C_{2}$ occurs without this consideration.

Now we deform the integral onto these contours. We illustrate this with the 
integral $J_{1}$ from above.

$$
\begin{aligned}
J_{1} & =\int_{a}^{b} e^{-\frac{1}{\sqrt{\delta}} \phi(s)} f^{-\frac{3}{4}}(s) g(s) d s, \\
& =\int_{C_{1}+C_{2}+C_{3}} e^{-\frac{1}{\sqrt{\delta}} \phi(s)} f^{-\frac{3}{4}}(s) g(s) d s, \\
& =\int_{0}^{l_{1}} e^{-\frac{1}{\sqrt{\delta}} \phi\left(c_{1}(t)\right)} f^{-\frac{3}{4}}\left(c_{1}(t)\right) g\left(c_{1}(t)\right) c_{1}^{\prime}(t) d t \\
& +\int_{0}^{l_{2}} e^{-\frac{1}{\sqrt{\delta}} \phi\left(c_{2}(t)\right)} f^{-\frac{3}{4}}\left(c_{2}(t)\right) g\left(c_{2}(t)\right) c_{2}^{\prime}(t) d t \\
& +\int_{0}^{l_{3}} e^{-\frac{1}{\sqrt{\delta}} \phi\left(c_{3}(t)\right)} f^{-\frac{3}{4}}\left(c_{3}(t)\right) g\left(c_{3}(t)\right) c_{3}^{\prime}(t) d t .
\end{aligned}
$$

The integrals along $C_{1}$ and $C_{3}$ are Laplace integrals (see section 4.1), with the maximum of $\phi\left(c_{1,3}\right)$ attained at one end point as they are steepest descent/ascent contours. We use Laplace's method to estimate these. The various terms in the integrand are represented with their Taylor series near the point $t=0$ where $-\phi\left(c_{1}\right)$ attains its maximum and $t=l_{3}$, where $-\phi\left(c_{3}\right)$ attains its maximum. We focus on $C_{1}$ below.

$$
\begin{aligned}
& \phi\left(c_{1}(t)\right) \approx \phi\left(c_{1}(0)\right)+t \phi^{\prime}\left(c_{1}(0)\right) c_{1}^{\prime}(0)+\ldots \\
& f^{-\frac{3}{4}}\left(c_{1}(t)\right) g\left(c_{1}(t)\right) c_{1}^{\prime}(t) \approx f^{-\frac{3}{4}}\left(c_{1}(0)\right) g\left(c_{1}(0)\right) c_{1}^{\prime}(0)+\ldots
\end{aligned}
$$

We require $c_{1}(0)$ and $c_{1}^{\prime}(0)$ to attain the leading order behaviour. From the integration limits we have that $c_{1}(0)=a$. Since $\phi\left(c_{1}(t)\right)$ has constant imaginary component, then $\frac{d}{d t}\left(\phi\left(c_{1}(t)\right)\right) \in \mathcal{R}$ and so

$$
\begin{aligned}
& \phi^{\prime}\left(c_{1}(t)\right) c_{1}^{\prime}(t) \in \mathcal{R}, \\
& \operatorname{Arg}\left(\phi^{\prime}\left(c_{1}(t)\right)\right)=-\operatorname{Arg}\left(c_{1}^{\prime}(t)\right), \\
& \operatorname{Arg}\left(c_{1}^{\prime}(0)\right)=-\operatorname{Arg}\left(\sqrt{\frac{i}{f(a)}}\right)=-\frac{\pi}{4} .
\end{aligned}
$$

Hence we have $c_{1}^{\prime}(0)=e^{-i \frac{\pi}{4}}$. This is enough to get the leading order expansion. Substituting in the value of $c_{1}^{\prime}(0)$ into the Taylor expansion of $\phi\left(c_{1}(t)\right)$ we get

$$
\begin{aligned}
\phi\left(c_{1}(t)\right) & \approx a+t \sqrt{\frac{i}{f(a)}} e^{-i \frac{\pi}{4}} \\
& \approx a+t \sqrt{\frac{1}{f(a)}} .
\end{aligned}
$$


And so the integral along $C_{1}$ is approximated by

$$
\begin{array}{rlrl}
\int_{C_{1}} & \sim \int_{0}^{\infty} e^{-\frac{1}{\sqrt{\delta}}\left(a+t \frac{1}{\sqrt{f(a)}}\right)} f^{-\frac{3}{4}}(a) g(a) e^{-i \frac{\pi}{4}} d t, & & \delta \rightarrow 0 \\
& \sim-\sqrt{\delta} f^{-\frac{3}{4}}(a) g(a) e^{-i \frac{\pi}{4}} e^{-\gamma(a)} \frac{1}{-\frac{1}{\sqrt{f(a)}}}, & & \delta \rightarrow 0 \\
& \sim \sqrt{\frac{\delta}{i}} f^{-\frac{1}{4}}(a) g(a) e^{-\gamma(a)}, & \delta \rightarrow 0 .
\end{array}
$$

This is the first surface term that arises if one uses integration by parts. Following a similar process we see that the contribution from $C_{3}$ gives the second surface term attained by integration by parts.

$$
\int_{C_{3}} \sim-\sqrt{\frac{\delta}{i}} f^{-\frac{1}{4}}(b) g(b) e^{-\gamma(b)} .
$$

Combining all the elements together, the leading order behaviour of the integral $J_{1}$ (equation 4.27) is as follows.

$$
J_{1} \sim \sqrt{\frac{\delta}{i}} f^{-\frac{1}{4}}(a) g(a) e^{-\gamma(a)}-\sqrt{\frac{\delta}{i}} f^{-\frac{1}{4}}(b) g(b) e^{-\gamma(b)}, \delta \rightarrow 0 .
$$

Although this is the same result as would have been obtained via integration by parts, the method of steepest descent justifies retaining both surface terms which cannot be justified otherwise. Similarly we compute the leading order behaviour of the other three integrals (equations 4.28,4.30) as follows.

$$
\begin{array}{lll}
J_{2} \sim \sqrt{\frac{\delta}{i}} f^{-\frac{1}{4}}(b) g(b) e^{\gamma(b)}-\sqrt{\frac{\delta}{i}} f^{-\frac{1}{4}}(a) g(a) e^{\gamma(a)} & , \delta \rightarrow 0, \\
J_{3} \sim \frac{\sqrt{\delta}}{\sqrt{i}+\frac{\sqrt{f(a)}}{\nu_{1}}} f^{-\frac{1}{4}}(a) g(a) e^{-\gamma(a)}-\frac{\sqrt{\delta}}{\sqrt{i}+\frac{\sqrt{f(b)}}{\nu_{1}}} f^{-\frac{1}{4}}(b) g(b) e^{-\gamma(b)} & , \delta \rightarrow 0, \\
J_{4} \sim \frac{\sqrt{\delta}}{\sqrt{i}-\frac{\sqrt{f(b)}}{\nu_{1}}} f^{-\frac{1}{4}}(b) g(b) e^{\gamma(b)}-\frac{\sqrt{\delta}}{\sqrt{i}-\frac{\sqrt{f(b)}}{\nu_{1}}} f^{-\frac{1}{4}}(a) g(a) e^{\gamma(a)} & , \delta \rightarrow 0 .
\end{array}
$$

\section{Approximate Solution using the Method of Steepest Descent}

Using the integrals approximated using the method of steepest descent, we turn to approximate the integrals that give rise to the form of $W(z)=W_{1}(z)+W_{2}(z)$. These have to be separated over two regions, $z<z_{c}$ and $z>z_{c}$.

$$
\begin{aligned}
& \mathbf{z}<\mathbf{z}_{\mathbf{c}}: \mathbf{z} \\
& \\
& \tilde{I}_{1} \sim J_{3}\left(z, z_{c}\right)+J_{1}\left(z_{c}, 1\right), \\
& \\
& I_{2} \sim J_{4}(0, z), \\
& \tilde{I}_{2} \sim J_{3}(0, z) .
\end{aligned}
$$


Substituting the above integrals into equations 4.22 and 4.23 , the following approximations are attained for $W_{1}(z)$ and $W_{2}(z)$, with appropriate approximations made for neglecting small terms in the last line.

$$
\begin{aligned}
& W_{1} \sim \sqrt[4]{\mathrm{f}(z)}\left(e^{\gamma(z)}+\beta e^{\gamma(z)}\right)\left(\frac{1}{2 \sqrt{i \delta}}\right) \tilde{I}_{1} \\
& \sim \frac{1}{2 \sqrt{i \delta}} \sqrt[4]{\mathrm{f}(z)}\left(e^{\gamma(z)}+\beta e^{-\gamma(z)}\right)\left(\frac{\sqrt{\delta}}{\sqrt{i}+\frac{\sqrt{f(z)}}{\nu_{1}}} F_{-}(z)\right. \\
& \left.-\frac{\sqrt{\delta}}{\sqrt{i}+\frac{\sqrt{f\left(z_{c}\right)}}{\nu_{1}}} F_{-}\left(z_{c}\right)+\sqrt{\frac{\delta}{i}} F_{-}\left(z_{c}\right)-\sqrt{\frac{\delta}{i}} F_{-}(1)\right) \\
& \sim \frac{1}{2} \frac{1}{i+\frac{\sqrt{i f(z)}}{\nu_{1}}} g(z)\left(1+\beta e^{-2 \gamma(z)}\right) \\
& +\frac{1}{2}\left(\frac{1}{i}-\frac{1}{i+\frac{\sqrt{i f\left(z_{c}\right)}}{\nu_{1}}}\right) \frac{f^{\frac{1}{4}}(z)}{f^{\frac{1}{4}}\left(z_{c}\right)} g\left(z_{c}\right) e^{-\gamma\left(z_{c}\right)}\left(e^{\gamma(z)}+\beta e^{-\gamma(z)}\right), \\
& W_{2} \sim \frac{1}{2 \sqrt{i \delta}} f^{\frac{1}{4}}(z) e^{-\gamma(z)}\left(I_{2}+\beta \tilde{I}_{2}\right) \\
& \sim \frac{1}{2 \sqrt{i \delta}} f^{\frac{1}{4}}(z) e^{-\gamma(z)}\left(\frac{\sqrt{\delta}}{\sqrt{i}-\frac{\sqrt{f(z)}}{\nu_{1}}} F_{+}(z)-\frac{\sqrt{\delta}}{\sqrt{i}-\frac{\sqrt{f(0)}}{\nu_{1}}} F_{+}(0)\right. \\
& \left.+\beta\left(\frac{\sqrt{\delta}}{\sqrt{i}+\frac{\sqrt{f(0)}}{\nu_{1}}} F_{-}(0)-\frac{\sqrt{\delta}}{\sqrt{i}+\frac{\sqrt{f(z)}}{\nu_{1}}} F_{-}(z)\right)\right) \\
& \sim \frac{1}{2 \sqrt{i}} f^{\frac{1}{4}}(z) e^{-\gamma(z)}\left(\frac{1}{\sqrt{i}-\frac{\sqrt{f(z)}}{\nu_{1}}} F_{+}(z)-\frac{1}{\sqrt{i}-\frac{1}{\nu_{1}}}\right. \\
& \left.+\beta\left(\frac{1}{\sqrt{i}+\frac{1}{\nu_{1}}}-\frac{1}{\sqrt{i}+\frac{\sqrt{f(z)}}{\nu_{1}}} F_{-}(z)\right)\right),
\end{aligned}
$$

where we have defined $F_{ \pm}(x)=f^{-\frac{1}{4}}(x) g(x) e^{ \pm \gamma(x)}$ for compactness.

$\mathrm{z}>\mathrm{z}_{\mathrm{c}}$ :

$$
\begin{aligned}
\tilde{I}_{1} & \sim J_{1}(z, 1) \\
I_{2} & \sim J_{4}\left(0, z_{c}\right)+J_{2}\left(z_{c}, z\right), \\
\tilde{I}_{2} & \sim J_{3}\left(0, z_{c}\right)+J_{1}\left(z_{c}, z\right) .
\end{aligned}
$$

Written explicitly, we are especially interested in the solution not close to $z=1$, 
and simplifications in this case are written from the third line.

$$
\begin{aligned}
& W_{1} \sim \sqrt[4]{\mathbf{f}(z)}\left(e^{\gamma(z)}+\beta e^{-\gamma(z)}\right)\left(\frac{1}{2 \sqrt{i \delta}}\right) \tilde{I}_{1} \\
& \sim \sqrt[4]{\mathrm{f}(z)}\left(e^{\gamma(z)}+\beta e^{-\gamma(z)}\right)\left(\frac{1}{2 \sqrt{i \delta}}\right) \sqrt{\frac{\delta}{i}}\left(F_{-}(z)-F_{-}(1)\right) \\
& \approx-\frac{i}{2} g(z) \text {. } \\
& W_{2} \sim \frac{1}{2 \sqrt{i \delta}} f^{\frac{1}{4}}(z) e^{-\gamma(z)}\left(I_{2}+\beta \tilde{I}_{2}\right) \\
& \sim \frac{1}{2 \sqrt{i \delta}} f^{\frac{1}{4}}(z) e^{-\gamma(z)}\left[\frac{\sqrt{\delta}}{\sqrt{i}-\frac{\sqrt{f\left(z_{c}\right)}}{\nu_{1}}} F_{+}\left(z_{c}\right)-\frac{\sqrt{\delta}}{\sqrt{i}-\frac{1}{\nu_{1}}}+\sqrt{\frac{\delta}{i}}\left(F_{+}(z)-F_{+}\left(z_{c}\right)\right)\right. \\
& \left.+\beta\left(\frac{\sqrt{\delta}}{\sqrt{i}+\frac{1}{\nu_{1}}}-\frac{\sqrt{\delta}}{\sqrt{i}+\frac{\sqrt{f\left(z_{c}\right)}}{\nu_{1}}} F_{-}\left(z_{c}\right)+\sqrt{\frac{\delta}{i}}\left(F_{-}\left(z_{c}\right)-F_{-}(z)\right)\right)\right] \\
& \approx-\frac{i}{2} g(z)-\frac{i}{2} f^{\frac{1}{4}}(z) e^{-\gamma(z)}\left[\left(\frac{1}{1-\sqrt{\frac{f\left(z_{c}\right)}{i \nu_{1}^{2}}}}-1\right) F_{+}\left(z_{c}\right)-\frac{1}{1-\frac{1}{\sqrt{i \nu_{1}}}}+\frac{\beta}{1+\frac{1}{\sqrt{i \nu_{1}}}}\right] \text {. }
\end{aligned}
$$

Using the definition of $\gamma(z)=\int_{0}^{z} \sqrt{\frac{i}{\delta f(x)}} d x$, we see that the term $\exp (-\gamma(z))$ is a decaying travelling thermal wave. For $z>z_{c}$ the approximate solution is explicitly a sum of travelling and stationary waves as the term in square brackets of the above equation is a constant with respect to $z$. For $z<z_{c}$ we have a sum of a travelling decaying wave, a reflected thermal wave from $z=z_{c}$, and a stationary wave. This has the same structure as the outer and inner solutions (equation 4.6. 4.8), but with the travelling waves taking on the form of the leading order WKB approximation to the homogeneous equation (equation 3.18). Numerical comparisons of this approximation are seen in Figures 4.7 and 4.8 . The approximation begins to misrepresent the solution significantly for large values of $\delta$ or relatively steep forms of $g(z)$, but otherwise are in close agreement with the numerical solutions.

Equations 4.354 .38 give a uniform approximation for the leading order behaviour of the thermal waves in terms of purely elementary functions. The analytical structure of these thermal waves may be studied further using these expressions, for example, in investigating the change of wave-type location and its behaviour. From values of $W$ we may calculate the corresponding temperature oscillations via $\epsilon \theta_{1}=\frac{1}{2 \omega} Q(0) U(z) W(z) e^{i \omega t}$ with $U(z)=\sqrt{\frac{k(0)}{k(z)}}$. 

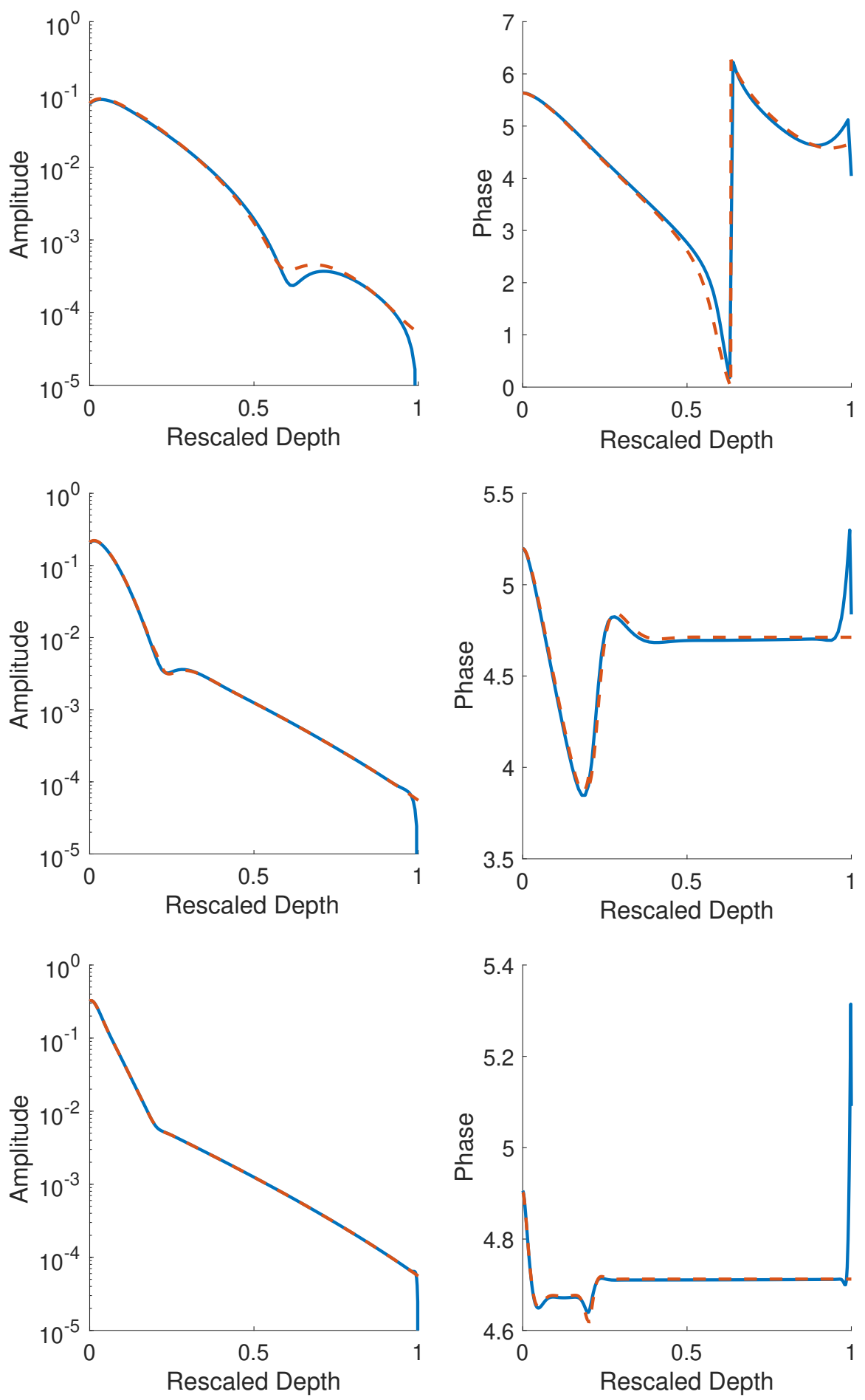

Figure 4.7: The approximation given in equations 4.35, 4.36, 4.37 and 4.38 (dashed, red) and numerical solutions to equation 3.13 (solid, blue) for the following parameter values: $\alpha=10, v_{1}=0.05, v_{2}=0.25, z_{c}=0.2, \delta=10^{-2}$ (top) $10^{-3}$ (middle) $10^{-4}$ (bottom). 

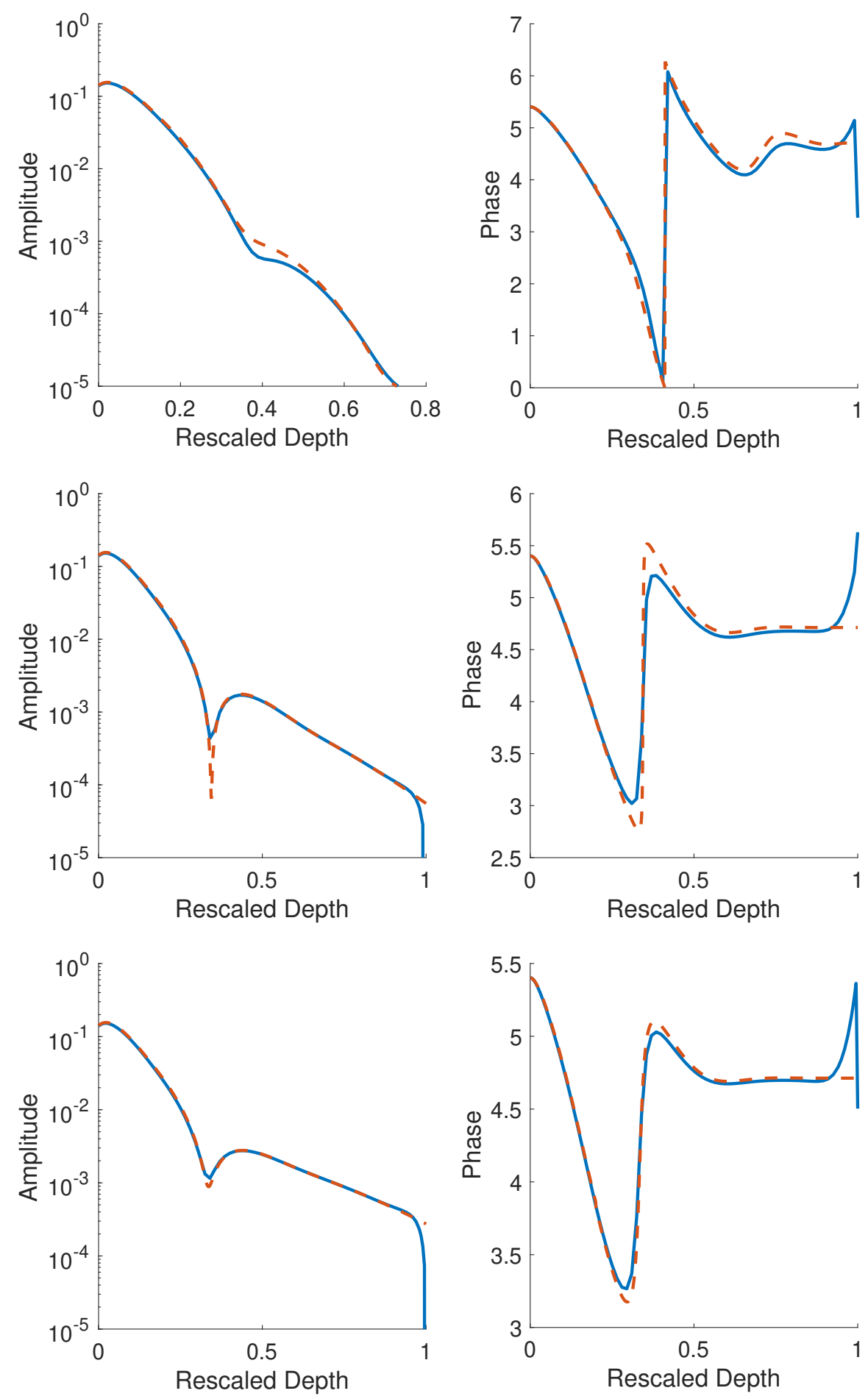

Figure 4.8: The approximation given in equations 4.35, 4.36, 4.37 and 4.38 (dashed, red) and numerical solutions to equation 3.13 (solid, blue) for the following parameter values: $\alpha=10, \delta=3 \times 10^{-3}, v_{1}=0.05, z_{c}=0.2, v_{2}=0.1$ (top) 0.25 (middle) 0.5 (bottom). 


\subsection{Extensions to any Time-Dependent Diurnal Source}

In the above analysis we took the time dependence of the solar radiation to be that of an oscillatory sine wave. This is a reasonably good assumption in McMurdo Sound, Antarctica in mid-November (see Figure 3.4) but would fail to capture the time dependence of early season light as the sun just barely begins to emerge. Inspired by the discussion presented by [37], the above formulation may be extended easily to analyse thermal waves of different time dependencies. This extension makes use of the key assumption that on a short time scale of a few days, the time-parameterization of the solar irradiance does not change significantly, meaning that it can be approximated by a Fourier series.

$$
Q(z, t)=Q(z) \times\left(a_{0}+\sum_{n \neq 0} a_{n} e^{i n \omega_{0} t^{+}}\right),
$$

where we assume that locally the function has period one day. Applying the two time analysis method as before we arrive at equation 3.7 with the source term containing the sum of frequencies. Its solution may be written as a sum of components, each taking into account a different term in the Fourier series.

$$
\begin{aligned}
& \theta_{1}(z, t)=\sum_{n \neq 0} \theta_{1}^{n}(z) e^{i n \omega_{0} t^{+}} \\
& i n \omega_{0} \theta_{1}^{n}=\frac{1}{\rho c} \frac{\partial}{\partial z}\left(k \frac{\partial \theta_{1}^{n}}{\partial z}\right)+\frac{a_{n}}{2} Q(z) e^{i n \omega_{0} t^{+}}
\end{aligned}
$$

As before this induces the oscillatory time dependence of $e^{i n \omega_{0} t^{+}}$on $\theta_{1}^{n}$ and hence we eventually get to equation 3.13 but with $\delta=\frac{1}{n \omega_{0}} D(0)$ and $V=\frac{a_{n}}{n \omega_{0}} Q(0) W(z)$, analogous to the parameterizations after equation 3.12. In most cases $a_{n}$ decreases rapidly enough that only a few terms are needed to represent the solution sufficiently.

For higher harmonics with increasing $n$, the value of $\delta$ gets smaller meaning that the outer solution becomes a good approximation of $\theta_{n}$ for $n>n_{0}$ for some $n_{0}$. The prefactor $\frac{a_{n}}{n}$ of the solution indicates that for most cases the contributions from harmonics of higher frequency becomes negligible. For example, the outer solution (the stationary waves) is given by

$$
\theta_{1, \text { outer }}\left(z, t^{+}\right)=-i Q(z) \sum_{n \neq 0} \frac{a_{n}}{n} e^{i \omega_{0} n t^{+}}
$$

with faster oscillating components contributing less to the solution. 


\subsection{Summary}

The equation for the leading behaviour of the thermal waves induced by the solar heating and oscillating air temperatures as derived in the previous chapter have been analysed using matched asymptotic techniques as well as the WKB method. The leading order WKB behaviour has been approximated in terms of elementary functions and shows clear evidence of the travelling and stationary wave behaviour seen in measurements. Comparisons with numerical solutions and numerical simulations are in good agreement with the asymptotic analysis.

The first two terms of the WKB approximation to equations 3.13 and 3.18 are seen to re-create numerical solutions for parameter values of physical interest accurately. Further, we approximate the WKB approximation (written in terms of integrals that may not be evaluated analytically) in terms of elementary functions by making use of the method of steepest descent. These also re-create the solutions/WKB approximation well for physically relevant parameters and converge to the exact (numerical) solution in the limit $\delta \rightarrow 0$. We show this with a number of numerical examples.

The approximated form of the WKB expansion has strong physical interpretations in terms of travelling and stationary waves and may be used to further study the analytical structure of these and the destructive interference that is sometimes present in the numerical solutions and data. Further investigations could investigate the changeover location between the stationary and travelling thermal waves or the solid-state greenhouse effect present near the surface. 


\section{Chapter 5}

\section{Soil Thermodynamics}

Soils are in-homogeneous materials and, similarly to sea ice, are commonly studied with thermistor string measurements $[30,62,76,80]$. They are porous media with complicated physical structures allowing water transport in liquid and/or vapour form. Advective heat transport is significant in soils, whereas solar radiative heating is only present at the surface of the soil.

There are two mathematical problems to solve, the forward problem: estimating soil temperatures based on measurements or knowledge of the soil parameters and water flows, and the inverse problem: estimating the soil parameters based on temperature measurements. These have been investigated numerically in some detail. Analytical analysis of these have been mainly limited to homogeneous soils with constant, uniform water flow [96]. Under these conditions the inverse problem [62] and the forward problem [33, 96] have been solved and implemented by various authors. The challenge still remains for development of analytical analysis for when the homogeneous soil with constant, uniform water flow assumptions are not met.

The WKB approximation for the temperature oscillations in sea ice suggests that similar approaches may be used in analysing the thermodynamics of soils. In this chapter we will briefly motivate studying thermodynamics in soils with reference to measurements presented by $[7]$ and common analysis tools. A similar method to Chapters 3 and 4 is used to derive the leading order behaviour of temperature oscillations due to the macroscale heterogeneity in soils. Using these results we demonstrate the effects of a depth varying thermal conductivity and the errors introduced by assuming a constant, average thermal conductivity. 


\subsection{Introduction}

In the past few decades using heat as a tracer for groundwater movement has aided studies of groundwater-surface water interactions; it has the benefit of being relatively cheap and easy to measure. Most methods developed for analysing temperature timeseries data and extracting estimates for soil diffusivity and seepage velocity are based on the theory of homogeneous soils. Amongst these include analytical [30,62, 65, 113], numerical [42,79, 85] and graphical [7, 93] tools, to give a few examples. Soils are inherently heterogeneous due to their composition and structure. The heterogeneity may be modelled on two length scales, the microscopic scale due to the presence of different materials and grain sizes, and the macroscopic scale, due to the composition of the materials varying with depth.

There has been a growing interest in determining the sensitivity and errors of the analysis methods developed for one dimensional, homogeneous soils with constant, uniform seepage velocity. For example, numerical studies have investigated the effects of time variations in the seepage velocity [81], heterogeneous soils [84, 86, 90] including heterogeneous hydraulic conductivity [41] and varying water flows [68]. Measurements of the thermal conductivity of soils by [86] and numerical simulations demonstrated that using in-stu measured thermal conductivity in modelling resulted in a seepage flux estimate up to $64 \%$ lower or $75 \%$ higher than when standard thermal conductivity values from the literature are adopted. Layered media were considered by [48, 89] where in each layer the homogeneous assumptions hold, and the resulting medium is shown to have interesting thermal wave propagation behaviour. In [37] it is demonstrated that the depth variation of the soil parameters introduces complicated structure to the amplitude and phase behaviour.

\subsubsection{Temperature Time Series Analysis Methods}

From measurements of the amplitude decay $(\ln (A))$ and phase shift $(\Delta \phi)$ of a particular harmonic signal between two thermistors at different depths, it is common to estimate the diffusivity and the seepage velocity. Most methods, with notable exceptions being that developed by [37] and some numerical studies such as [86], are based on homogeneous soils.

Based on the Stallman equation derived by [96], being the solution to the constant parameters advection-diffusion equation, Hatch et al. [30] derived transcendental formulae to estimate the thermal front velocity, and hence the seepage velocity based on measurements of the amplitude decay and phase shift of a har- 
monic temperature signal propagating into the soil. These are given by

$$
\begin{aligned}
v_{t, A} & =\frac{2 \kappa_{e}}{\Delta z} \ln (A)+\sqrt{\frac{\alpha+v_{t, A}^{2}}{2}}, \\
v_{t, \phi} & =\sqrt{\alpha-2\left(\frac{\Delta \phi 4 \pi \kappa_{e}}{P \Delta z}\right)^{2}},
\end{aligned}
$$

where $\alpha=\sqrt{v_{t}^{4}+\left(\frac{8 \pi \kappa_{e}}{P}\right)^{2}}$ with $v_{t}$ taking on the appropriate subscript for each equation, $\kappa_{e}$ is the effective thermal diffusivity and $A, \Delta \phi$ are the amplitude ratio and phase shift of the detected signal between the two thermistors of interest, spaced $z \mathrm{~m}$ apart. $v_{t}$ is the thermal front velocity defined as $v_{t}=q \frac{\rho_{w} c_{w}}{\rho c}$ with $q$ being the vertical seepage velocity, $\rho$ and $c$ are the density and specific heat capacity of the soil, and similarly $\rho_{w}$ and $c_{w}$ are the density and specific heat capacity of water (or the fluid flowing through the soil). It is important to note that the effective thermal diffusivity, $\kappa_{e}$, must be estimated prior to applying these formula. [65] and then [62] demonstrated that these expressions may be combined for a simultaneous estimation of the thermal diffusivity and the seepage velocity using a direct equation. The formula presented by [62] are as follows

$$
\begin{aligned}
\kappa_{e, \text { measured }} & =\frac{\eta z^{2} \omega}{\ln ^{2}(A)+\Delta \phi^{2}}, \\
v_{t, \text { measured }} & =\frac{\omega \Delta z}{\sqrt{\ln ^{2}(A)+\Delta \phi^{2}}} \frac{1-\eta^{2}}{\sqrt{1+\eta^{2}}},
\end{aligned}
$$

with $\eta=-\frac{\ln (A)}{\Delta \phi}$.

Graphical methods, such as finite difference schemes used by [76], fourier analysis methods [7] or thermal orbits [93], are also based on the solution to the homogeneous soil with constant uniform seepage velocity. Further, numerical tools such as [42,85] make use of the above mentioned analytical tools to estimate the soil parameters and seepage velocity. Other numerical tools such as presented by [79] use the constant parameter solution to estimate the average parameters between thermistors.

\subsubsection{Data Analysis}

Here we present some data analysis to illustrate some of the above mentioned methods. We use the data provided by [7], available at [6]. This is a thermistor string with thermistors at depths of $0.1 \mathrm{~m}, 0.3 \mathrm{~m}, 0.5 \mathrm{~m}, 0.7 \mathrm{~m}, 0.9 \mathrm{~m}$ and $1.1 \mathrm{~m}$, planted in the Carrapateena mine site in South Australia. Temperatures at these 
depths were recorded every 15 minutes from December 2012 until August 2013. The temperature record is presented in Figure 5.1. Using the methods described in [7], the timeseries of each thermistor is processed using a Fast Fourier Transform, with the results shown in Figure 5.1. We clearly see a diurnal signal at all depths.

The theory for homogeneous soils with no advective heat transport predicts exactly equal depth variations of $\ln (A)$ and $\Delta \phi[37]$, being the amplitude decay and phase shift of a harmonic temperature oscillation. The measured $\ln (A)$ and $\Delta \phi$ for the diurnal signal are shown in Figure 5.2. A slight non-linear behaviour over depth is observed for both $\ln (A)$ and $\Delta \phi$, as well as a discrepancy between the two indicating the presence of non-conductive heat transport and heterogeneous parameters. Figure 5.3 shows plots of $\ln (A)$ and $\Delta \phi$ between every consecutive pair of thermistors as a function of frequency. It is evident that the behaviours of the two quantities are not the same, and the greatest discrepancy occurs at the surface where we expect the most non-conductive heat transport to occur.

Further, one may apply equations 5.1 and 5.2 to each harmonic in the fourier series to estimate the diffusivity and seepage velocity. The results of this are presented in Figure 5.4. In a homogeneous soil with constant water flow, we expect the values plotted in Figure 5.4 to not depend on frequency, being the harmonic of the fourier decomposition of the temperature records that was used for analysis. However, it is evident that there is a frequency dependence of these measurements, especially in the seepage flux estimates. This indicates a significant violation of the model assumptions, a time variation in the water flux and/or depth dependent parameters. The diffusivity estimates are within agreement of the estimates described in [7], with an expected increase in thermal diffusivity between the last pair of thermistors.

\subsubsection{Non-Uniform Soils}

Attention to the effects of the non-uniformity of soil parameters with only conductive effects was considered by [37] and has been applied by numerous authors, for example [76]. A one dimensional model is considered, neglecting horizontal heat transport. That is, we assume that there are no macroscopic horizontal variations in the soil properties and water flow, hence no heat flows horizontally. With depth dependent thermal conductivity and heat capacity, the heat equation is assumed to have a solution of the form $T(z, t)=T_{0}(z)+\sum r_{L}(z) \sin \left(L \omega t+\phi_{L}(z)\right)$, interpreted as $r_{L}$ and $\phi_{L}$ being the amplitude and phase of the harmonic with an- 

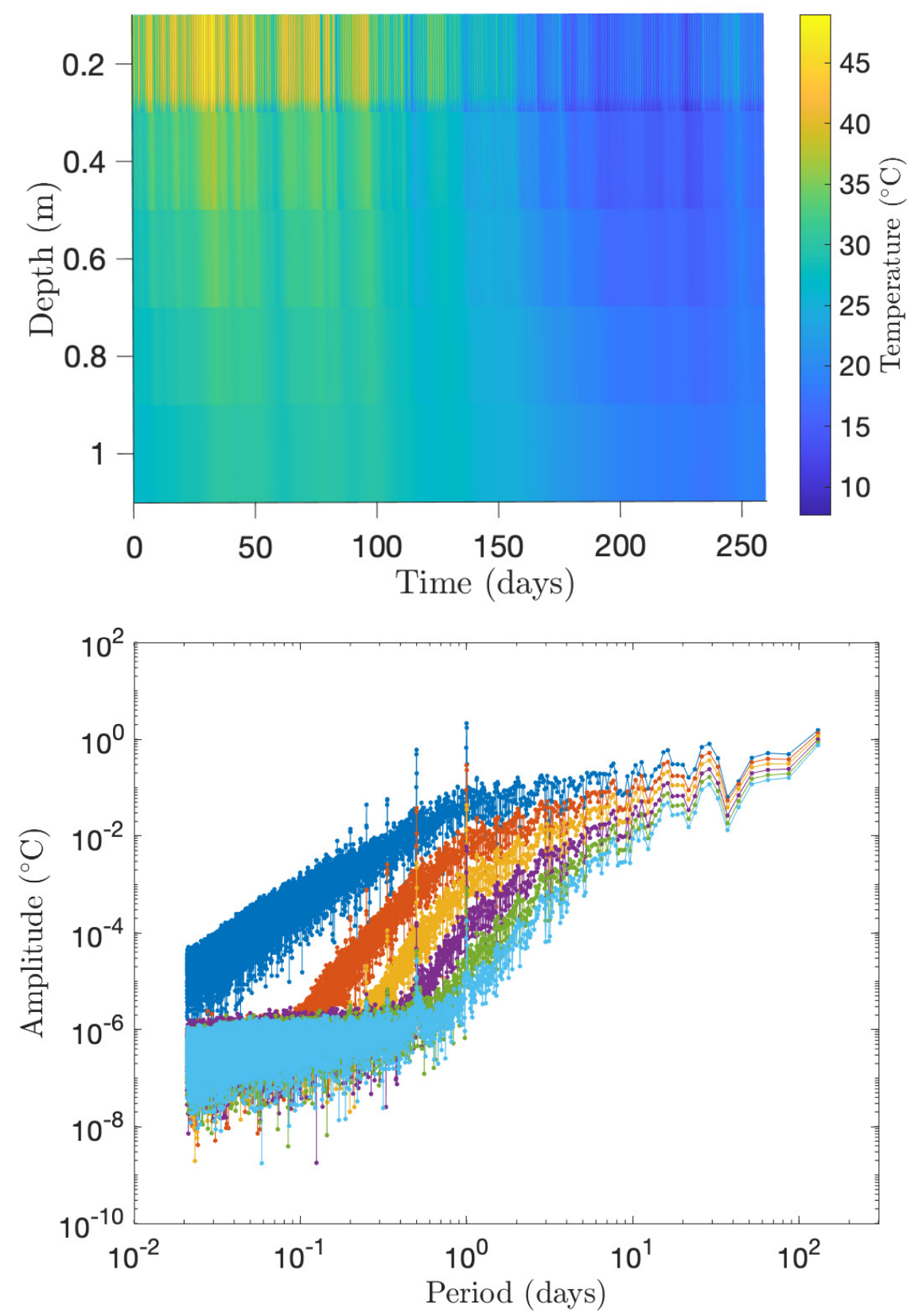

Figure 5.1: The Carrapateena site temperature record presented by [7]. Top: Temperature is plotted as the vertical axis and shown via the colourmap $\left({ }^{\circ} \mathrm{C}\right)$. Bottom: The Fast Fourier Transform of the timeseries of each thermistor plotted against the period of oscillation. The thermistor depths correspond to the colours: Dark blue - $0.1 \mathrm{~m}$. Red - $0.3 \mathrm{~m}$. Orange 0.5 m. Purple - 0.7 m. Green - 0.9 m. Cyan - 1.1 m. 


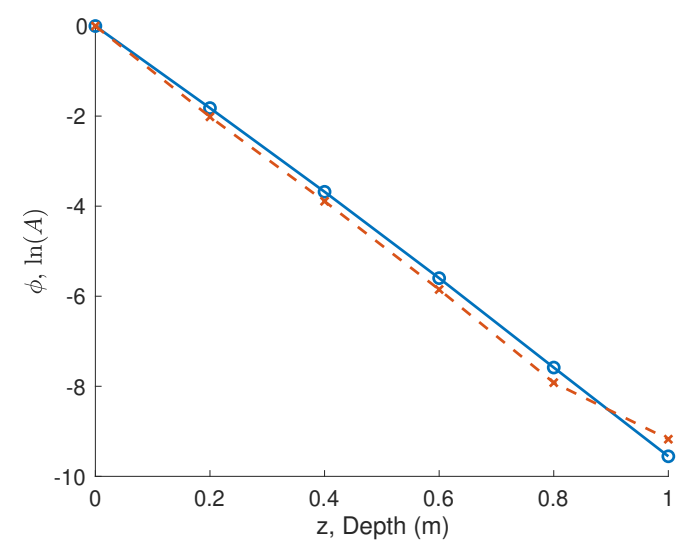

Figure 5.2: $\ln (A)$ (dashed red line, $x^{\prime}$ s) and $\Delta \phi$ (solid blue, o's) of the diurnal signal vs depth in the Carrapateena site temperature record.

gular frequency $\omega L$. By plugging into the heat equation we arrive at the following formulae for the thermal properties

$$
\begin{aligned}
\frac{k^{\prime}}{k} & =\frac{r_{L} \phi_{L}^{\prime 2}-r_{L}^{\prime \prime}}{r_{L}^{\prime}}, \\
\frac{\omega L}{\kappa} & =\frac{2 r_{L}^{\prime} \phi_{L}^{\prime}}{r_{L}}+\phi_{L}^{\prime \prime}+\frac{k^{\prime} \phi_{L}^{\prime}}{k},
\end{aligned}
$$

where primes denote differentiation with respect to depth. For a uniform soil the first of the above equations will equal zero, whereas the second of the above equations will only include the first term on the right hand side. For a uniform soil these may be solved together to get $r_{L}=e^{-\alpha z}$ and $\phi_{L}=-\alpha z$ for a constant $\alpha$ which depends on the thermal diffusivity and $\omega L$. These formulae predict non-linear in depth behaviours for $\ln \left(r_{L}\right)$ and $\phi$, as seen in Figure 5.2. for depth depended soil parameters, as discussed by [37]. However the formula do not account for non-conductive heat transport such as advection due to vertical water movement.

\subsection{Differential Equation for Thermal Waves in Soils}

If we assume the soil has only depth dependent parameters, as in [37], one can immediately write down a linear advection-diffusion equation [2],

$$
\rho c \frac{\partial T}{\partial t}=\frac{\partial}{\partial z}\left(k \frac{\partial T}{\partial z}\right)-\frac{\partial}{\partial z}\left(\rho_{w} c_{w} q T\right),
$$

with $k$ being the effective thermal conductivity, $\rho c$ the volumetric heat capacity of the soil-water system, $\rho_{w} c_{w}$ is the volumetric heat capacity of the water and 


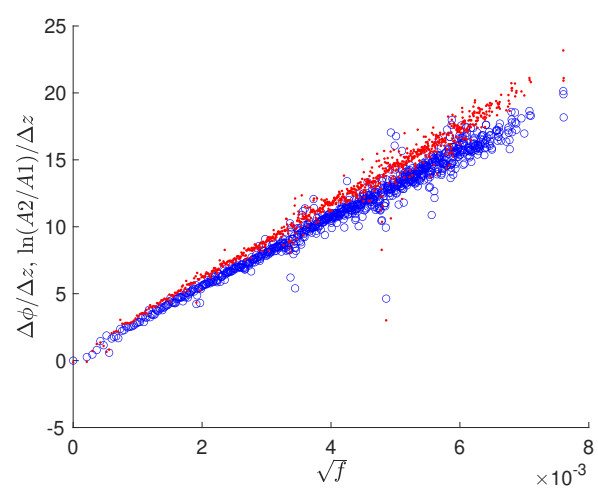

(a)

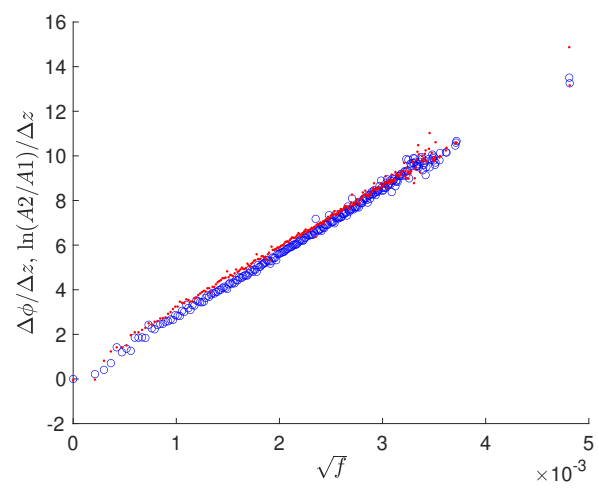

(c)

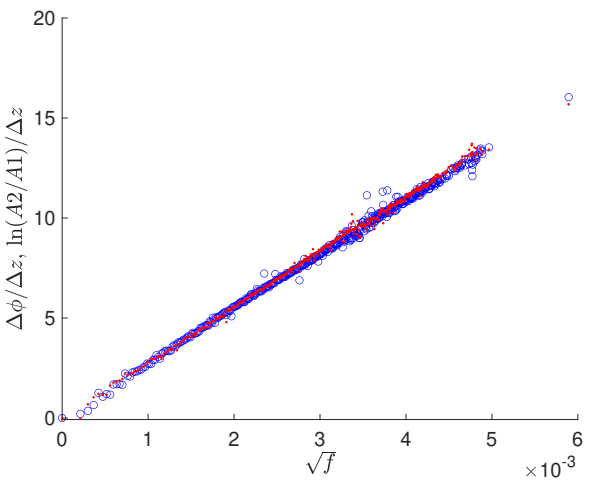

(b)

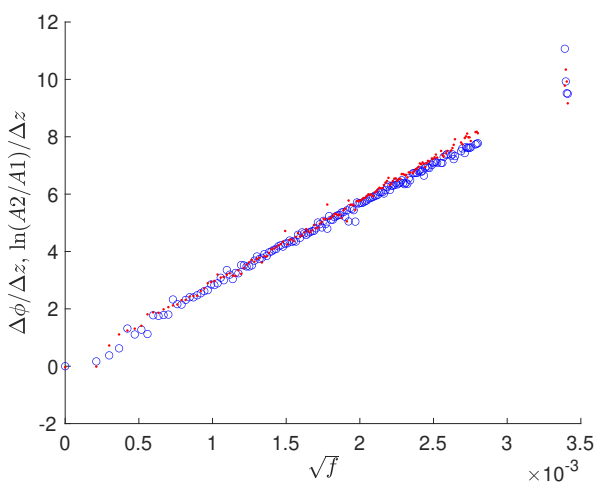

(d)

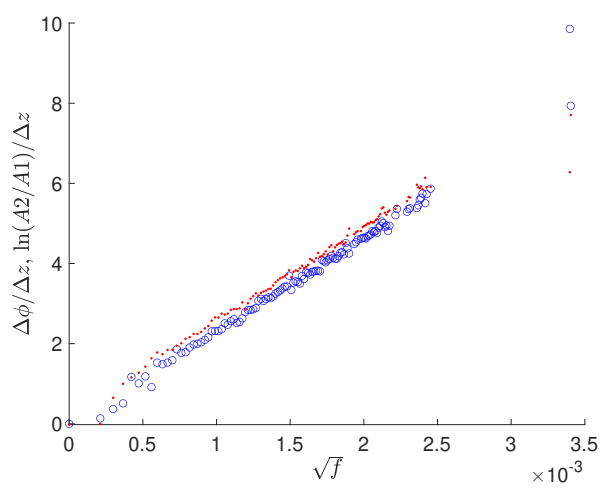

(e)

Figure 5.3: $\ln \left(A_{2} / A_{1}\right) / \Delta z$ (red dots) and $\Delta \phi / \Delta z$ (blue o's), between every set of consecutive thermistors, os square root of frequency for the Carrapateena site temperature record. The thermistors are: (a) $0.1 m-0.3 m$, (b) $0.3 m-0.5 m$, (c) $0.5 m-0.7 m,(d) 0.7 m$ $0.9 \mathrm{~m}$ and (e) $0.9 \mathrm{~m}-1.1 \mathrm{~m}$. 

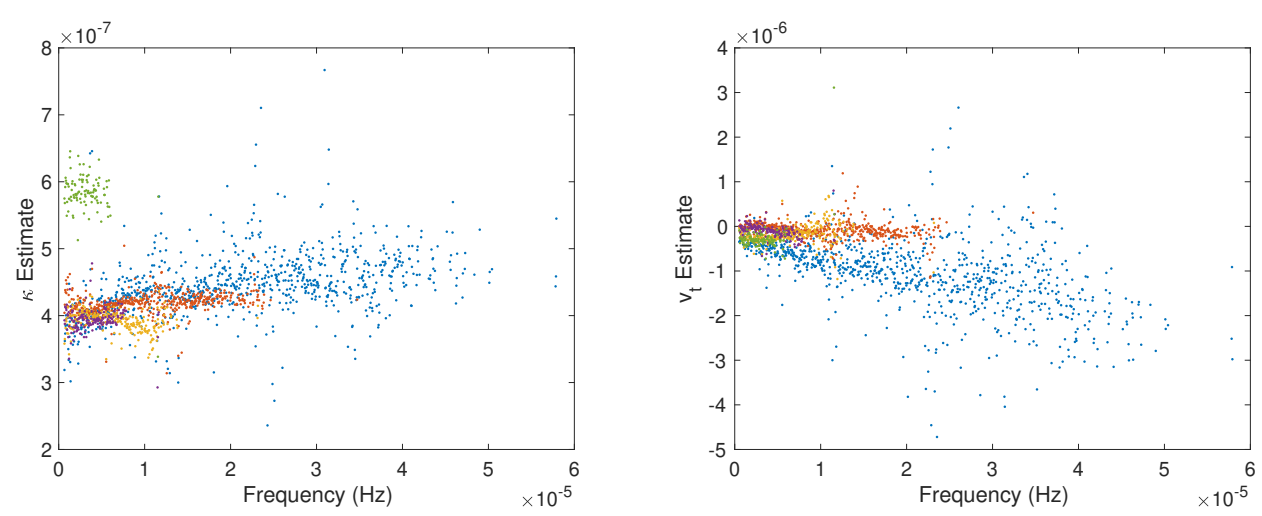

Figure 5.4: Applying equations 5.1 and 5.2 to the fourier decomposition of the Carrapateena site temperature record. The colours correspond to the following thermistors analysed: Blue - 0.1-0.3 m. Red - 0.3-0.5 m. Orange - 0.5-0.7 m. Purple-0.7-0.9 m. Green - 0.9-1.1 m.

$q$ is the vertical Darcian fluid velocity or seepage flux (positive is downwards). Standard boundary conditions from the literature are applied, a forced function of time at the upper surface, and a fixed temperature at infinity.

$$
\begin{array}{r}
T(0, t)=T_{\text {top }}(t), \\
\lim _{L \rightarrow \infty} T(L, t)=T_{\text {Earth }} .
\end{array}
$$

However, it is known that a temperature dependence is present in the thermal properties of soils, as measured for example by [60,76]. This makes the heat equation above practically impossible to solve. Having homogenized the equation over the microscale, a similar approach to that taken in the sea ice thermodynamics is used to find the leading order behaviour of the thermal waves of small amplitude and fast frequency.

We begin by non-dimensionalising the equation. Let $T=T_{0} \bar{T}, z=z_{0} \bar{z}, t=t_{0} \bar{t}$, $\rho c=\rho_{0} c_{0} \bar{\rho} \bar{c}, k=k_{0} \bar{k}$ and $\rho_{w} c_{w} q=\rho_{w, 0} c_{w, 0} q_{0} \bar{\rho}_{w} \bar{c}_{w} \bar{q}_{w}$, with subscript 0 indicating a constant. For common measurements, we let $T_{0}=40{ }^{\circ} \mathrm{C}, z_{0}=1 \mathrm{~m}, \rho_{0} c_{0}=$ $5 \times 10^{6} \mathrm{~J} /\left(\mathrm{C} \mathrm{m}^{3}\right), k_{e, 0}=1 \mathrm{~W} /(\mathrm{m}-\mathrm{C}), \rho_{w, 0} c_{w, 0} q_{0}=1 \mathrm{~J} /\left(\mathrm{C} \mathrm{m}^{2} \mathrm{~s}\right)$ [12, 17, 86]. We drop the bars on the variables and the advection-diffusion equation thus reads

$$
\rho c \frac{\partial T}{\partial t}=\frac{\partial}{\partial z}\left(k \frac{\partial T}{\partial z}\right)-\frac{\partial}{\partial z}\left(\rho_{w} c_{w} q T\right),
$$

where we have selected $t_{0}=\rho_{0} c_{0}=5 \times 10^{6}$. We apply a two time analysis following [45] by defining fast time $t^{+}=t+\mathcal{O}\left(\epsilon^{2}\right)$ and slow time $\tilde{t}=\epsilon t$. We set the upper boundary condition to have a sum of a slow time function and a fast time function, specifically assuming that the fast time dependence is of order $\epsilon$, while 
the slow time dependence is of order $1 . T_{\text {top }}=F_{1}(\tilde{t})+\epsilon F_{2}\left(t^{+}\right)$. Via the chain rule we have

$$
\frac{\partial}{\partial t}=\frac{\partial}{\partial t^{+}}+\epsilon \frac{\partial}{\partial \tilde{t}}+\mathcal{O}\left(\epsilon^{2}\right) .
$$

We apply an asymptotic expansion with $T\left(z, t^{+}, \tilde{t}\right)=T_{0}(z, \tilde{t})+\epsilon T_{1}\left(z, t^{+}, \tilde{t}\right)+$ $\mathcal{O}\left(\epsilon^{2}\right)$ and motivated by the data assume the background temperature, $T_{0}$, is independent of fast time. This allows us to expand the physical parameters (that depend on temperature and depth) around $T=T_{0}$, giving

$$
\begin{aligned}
k(z, T) & =k\left(z, T_{0}\right)+\epsilon T_{1} k_{T}\left(z, T_{0}\right)+\mathcal{O}\left(\epsilon^{2}\right), \\
\rho(z, T) & =\rho\left(z, T_{0}\right)+\epsilon T_{2} \rho_{T}\left(z, T_{0}\right)+\mathcal{O}\left(\epsilon^{2}\right), \\
c(z, T) & =c\left(z, T_{0}\right)+\epsilon T_{1} c_{T}\left(z, T_{0}\right)+\mathcal{O}\left(\epsilon^{2}\right),
\end{aligned}
$$

where subscript $T$ indicates partial differentiation with respect to $T$. From now on we drop the brackets on the physical parameters. We plug these expressions into the partial differential equation and collect terms of powers of $\epsilon$.

$$
\mathcal{O}\left(\epsilon^{0}\right): \quad \begin{aligned}
0 & =D \frac{\partial^{2} T_{0}}{\partial z^{2}}+\frac{\partial T_{0}}{\partial z}\left(-\gamma q+\frac{k^{\prime}}{\rho c}\right)-T_{0} \gamma q^{\prime} \\
\mathcal{O}\left(\epsilon^{1}\right): \quad \frac{\partial T_{0}}{\partial \tilde{t}}+\frac{\partial T_{1}}{\partial t^{+}} & =D \frac{\partial^{2} T_{1}}{\partial z^{2}}+\frac{\partial T_{1}}{\partial z}\left(-\gamma q+\frac{k^{\prime}}{\rho c}\right)-T_{1} \gamma q^{\prime} \\
& +T_{1}\left[\frac{\partial D}{\partial T} \frac{\partial^{2} T_{0}}{\partial z^{2}}+\frac{\partial T_{0}}{\partial z} \frac{\partial}{\partial T}\left(-\gamma q+\frac{k^{\prime}}{\rho c}\right)-T_{0} \frac{\partial \gamma}{\partial T} q^{\prime}\right],
\end{aligned}
$$

where' denotes partial differentiation with respect to $z$, and we have denoted $\gamma=\rho_{w} c_{w} /(\rho c)$ and $D=k /(\rho c)$. Notice that the temperature derivatives of the physical parameters are all present in the coefficient of the term $T_{1}$. Measurements by [60,76] indicate that this coefficient is at least two orders of magnitude smaller than the other coefficients of equation 5.9, so similarly to the sea ice, we omit the term with square brackets in equation 5.9. The powers of $\epsilon$ are matched in the boundary conditions.

$$
\begin{aligned}
\left.T_{0}\right|_{z=0} & =F_{1}(\tilde{t}), \\
\left.\lim _{L \rightarrow \infty} T_{0}\right|_{z=L} & =T_{\text {Earth }}, \\
\left.T_{1}\right|_{z=0} & =F_{2}\left(t^{+}\right), \\
\left.\lim _{L \rightarrow \infty} T_{1}\right|_{z=L} & =0 .
\end{aligned}
$$

Equation 5.8 may be solved for $T_{0}$ independently of $T_{1}$, although the non-linearity of equation 5.8 means that a numerical solution is generally required. For equation 5.9. we are particularly interested in the effects of the boundary conditions, 
i.e. the thermal waves induced by the fast time boundary conditions. We write the solution as a sum of two components, $T_{1}=\tilde{T}_{1}+\bar{T}_{1}$ where $\tilde{T}$ satisfies equation 5.9 including the term $\frac{\partial T_{0}}{\partial \tilde{t}}$ with a homogeneous boundary condition at $z=0$, and $\bar{T}$ satisfies the equation without the term $\frac{\partial T_{0}}{\partial \bar{t}}$ and with the non-homogeneous boundary condition at $z=0$.

$$
\begin{aligned}
\frac{\partial \bar{T}_{1}}{\partial t^{+}} & =D \frac{\partial^{2} \bar{T}_{1}}{\partial z^{2}}+\frac{\partial \bar{T}_{1}}{\partial z}\left(-\gamma q+\frac{k^{\prime}}{\rho c}\right)-\bar{T}_{1} \gamma q^{\prime}, \\
\left.\bar{T}_{1}\right|_{z=0} & =F_{2}\left(t^{+}\right), \\
\left.\lim _{L \rightarrow \infty} \bar{T}_{1}\right|_{z=L} & =0 . \\
\frac{\partial T_{0}}{\partial \tilde{t}}+\frac{\partial \tilde{T}_{1}}{\partial t^{+}} & =D \frac{\partial^{2} \tilde{T}_{1}}{\partial z^{2}}+\frac{\partial \tilde{T}_{1}}{\partial z}\left(-\gamma q+\frac{k^{\prime}}{\rho c}\right)-\tilde{T}_{1} \gamma q^{\prime}, \\
\left.\tilde{T}_{1}\right|_{z=0} & =0, \\
\left.\lim _{L \rightarrow \infty} \tilde{T}_{1}\right|_{z=L} & =0 .
\end{aligned}
$$

We interpret the term $\frac{\partial T_{0}}{\partial \tilde{t}}$ as a distributed source term with $\tilde{T}_{1}$ being the thermal waves generated by it and $\bar{T}_{1}$ as the thermal waves generated by the fast time boundary condition. $\tilde{T}_{1}$ has secular terms due to the source term being fast time independent, indicating that the expansion's validity is restricted to some point in fast time. We focus on $\bar{T}_{1}$ and drop the bar. It satisfies a linear partial differential equation, where the coefficients all depend on slow time and depth giving $T_{1}$ an implicit dependence on slow time. Equation 5.10 is separable and so we write the solution as

$$
T_{1}=\sum \Gamma_{n}\left(t^{+}\right) \theta_{n}(z)
$$

with

$$
\begin{aligned}
& \Gamma_{n}\left(t^{+}\right)=A_{n} e^{i \omega_{n} t^{+}}, \\
& D(z) \theta_{n}^{\prime \prime}+\left(\frac{k^{\prime}(z)}{\rho(z) c(z)}-\gamma(z) q(z)\right) \theta_{n}^{\prime}-\left(\gamma(z) q^{\prime}(z)+i \omega_{n}\right) \theta_{n}=0,
\end{aligned}
$$

with the boundary conditions

$$
\begin{aligned}
\sum_{n} \Gamma_{n}\left(t^{+}\right) & =F_{2}\left(t^{+}\right), \\
\theta_{n}(0) & =1, \\
\lim _{L \rightarrow \infty} \theta_{n}(L) & =0 .
\end{aligned}
$$

We see that the $\Gamma_{n}$ 's are exactly the components of the fourier expansion series of $F_{2}\left(t^{+}\right)$. The magnitude of $\theta_{n}(z)$ corresponds to the amplitude of the frequency 
$\omega_{n}$ whereas the phase corresponds to the lag of the frequency as a function of depth. We apply a change of variables in order to eliminate the first derivative term by letting $\theta_{n}(z)=U(z) V(z)$ with

$$
\begin{gathered}
U(z)=\exp \left(-\int_{0}^{z} \frac{k^{\prime}(x)-q(x) \rho_{w} c_{w}}{2 k(x)} d x\right) \\
D(z) V^{\prime \prime}(z)+V(z)\left(-i \omega_{n}-D(z)\left[\left(\frac{k^{\prime}(z)-q(z) \rho_{w} c_{w}}{2 k(z)}\right)^{\prime}\right.\right. \\
\left.\left.+\frac{q^{\prime}(z) \rho_{w} c_{w}}{k(z)}+\left(\frac{k^{\prime}(z)-q(z) \rho_{w} c_{w}}{2 k(z)}\right)^{2}\right]\right)=0 .
\end{gathered}
$$

Most researchers define the thermal front velocity as $v_{t}=\gamma(z) q(z)$ and report the solution in terms of this parameter. However, it is rather more convenient to define the parameter $\xi(z)=\left(q(z) \rho_{w} c_{w}-k^{\prime}(z)\right) /(2 k(z))$ being a reparameterization of the seepage velocity with reference to the effective thermal conductivity similar to that used by [44]. We also reparameterize the diffusivity using $\delta=D(0) / \omega_{n}$, $f(z)=D(z) / D(0)$ and denote $Q(z)=\xi^{2}-\xi^{\prime}+q^{\prime}(z) \rho_{w} c_{w} / k(z)$. The above equation re-arranges to the following

$$
\begin{aligned}
& U(z)=\exp \left(\int_{0}^{z} \xi(x) d x\right) \\
& \delta V^{\prime \prime}(z)-V(z)\left(\frac{i}{f(z)}+\delta Q(z)\right)=0 .
\end{aligned}
$$

We note here the effects of a varying thermal conductivity and the seepage velocity come into the equation in the same form, real components of $\xi$ and $Q$ that do not depend on the frequency of the thermal wave. From temperature measurements alone we cannot determine both the thermal properties and the seepage velocity.

We may dimensionalise this equation by reversing the re-parameterizations performed to non-dimensionalize equation 5.5. $D \rightarrow D /\left(\rho_{0} c_{0}\right), t \rightarrow t_{0} t$ and hence $\omega_{n} \rightarrow \omega_{n} / t_{0}$. These only appear in equation 5.15 in the term $\delta=D(0) t_{0} /\left(\omega_{n} \rho_{0} c_{0}\right)$ and cancel as the choice $\rho_{0} c_{0}=t_{0}$ was made. Hence equation 5.15 may be used with SI units for the parameters.

\subsubsection{General Approximate Solutions}

Equation 5.15 is a second order, complex valued differential equation with depth dependent coefficients, so just like with the sea ice thermodynamics, no closed 
form solution exists. However, asymptotic approximations such as the WKB expansion may be computed, as will be done moving forwards.

We make use of the small numerical value of the parameter $\delta$ in equation 5.15 to apply a singular perturbation. Here, one needs to quantify the relative sizes of the terms present in the above equation in order to proceed with a perturbation expansion. That is, one needs to specify the 'size' (being the order of $\delta$ ) of $Q(z)$ in equation 5.15. Measurements by [12,17] in riverbeds show common seepage velocities are on the scale of $q \approx 10^{-5} \mathrm{~m} / \mathrm{s}$. Using measurements of the diurnal cycle $\left(\omega \approx 7 \times 10^{-5} \mathrm{~s}^{-1}\right)$ and soil diffusivities of $D \approx 10^{-7} \mathrm{~m}^{2} / \mathrm{s}$, we get $\delta Q \approx$ $0.5 \sim i / f(z)$. We assume the dominant balance $Q(z) \sim 1 / \delta$ and specifically take $Q(z)=\tilde{Q}(z) / \delta$. We note that the WKB approximation is asymptotically still valid to leading order even when this assumption fails, for example, for a very low seepage velocity.

The leading order WKB behaviour of the solution is written as

$$
V(z) \sim \exp \left(\frac{1}{\sqrt{\delta}} S_{0}+S_{1}+\mathcal{O}(\sqrt{\delta})\right)
$$

with

$$
\begin{aligned}
& S_{0}= \pm \int_{0}^{z} \sqrt{\frac{i}{f(x)}+\tilde{Q}(x)} d x \\
& S_{1}=-\frac{1}{4} \ln \left(\frac{i}{f(z)}+\tilde{Q}(z)\right)+\frac{1}{4} \ln \left(\frac{i}{f(0)}+\tilde{Q}(0)\right) .
\end{aligned}
$$

As with the sea ice, this is a valid approximation if the next term, $\sqrt{\delta} S_{2}(z)$, is much smaller than 1 . This is the case if $f(z)$ does not have a zero.

The general approximate solution is written as a linear combination of the above expression, each with a different sign for $S_{0}$, however, the boundary condition at infinity requires that only the decaying solution is present. We note that for relevant parameters, the term $i / f(z)+\tilde{Q}(z)$ does not pass zero, making the above WKB approximation valid [9]. Combining with the solution for $U(z)$ via $\theta_{n}=V(z) U(z)$ and ensuring that the boundary condition at $z=0$ is met we get

$$
\begin{aligned}
\theta_{n}(z) \sim \exp [ & -\int_{0}^{z}\left(\frac{1}{\sqrt{\delta}} \sqrt{\frac{i}{f(x)}+\tilde{Q}(x)}-\xi(x)\right) d x \\
& \left.-\frac{1}{4} \ln \left(\frac{i}{f(z)}+\tilde{Q}(z)\right)+\frac{1}{4} \ln \left(\frac{i}{f(0)}+\tilde{Q}(0)\right)\right], \delta \rightarrow 0 .
\end{aligned}
$$

We note that for most functional forms of $f(x)$ and $\tilde{Q}(x)$, analytical expressions of such an integral do not exist or are written in terms of special functions. 


\subsubsection{Reduction to Homogeneous Soils with Constant, Uniform Seepage Velocity}

With $f_{\text {hom }}(z)=1, \xi_{\text {hom }}=q \rho_{w} c_{w} /(2 k)$ and $Q_{\text {hom }}(z)=\left(q \rho_{w} c_{w} /(2 k)\right)^{2}$, the solution to equation 5.16 is as follows

$$
\theta_{n, \mathrm{hom}}(z) \sim \exp \left(-\left(\sqrt{\frac{i}{\delta}+\xi^{2}}-\xi\right) z\right)
$$

Using trigonometric identities to separate the real and imaginary components one arrives at the equation

$\theta_{n, \text { hom }}(z) \sim \exp \left[-\frac{1}{\sqrt{2}}\left(\sqrt{\sqrt{\xi^{4}+\frac{1}{\delta^{2}}}+\xi^{2}}-\sqrt{2} \xi\right) z-\frac{i}{\sqrt{2}}\left(\sqrt{\sqrt{\xi^{4}+\frac{1}{\delta^{2}}}}-\xi^{2}\right) z\right]$.

These are exactly the Stallman equations [96] being the exact solution to the homogeneous soil with uniform water flow. The real part of the exponent dictates the amplitude decay of the temperature signal, whereas the imaginary component dictates its phase lag.

From these equations, one can set the real component of the exponent to $\ln (A)$ and the imaginary component to $\Delta \phi$ and get similar formulae for $\delta$ and $\xi$ to those in [62]. Doing so, we arrive at the following expressions.

$$
\begin{aligned}
\delta \omega=D & = \pm \frac{\ln \left(A_{r}\right) \omega z^{2}}{\Delta \phi\left(\Delta \phi^{2}+\ln \left(A_{r}\right)^{2}\right)} . \\
\xi & =\frac{\ln \left(A_{r}\right)^{2}-\Delta \phi^{2}}{2 \ln \left(A_{r}\right) z} .
\end{aligned}
$$

By the definition of $\xi=q \rho_{w} c_{w} /(2 k)$ and the variable $v_{t}=q \rho_{w} c_{w} /(\rho c)$ as defined in [62], we have $\xi=v_{t} /(2 \delta)$ and it is simple to show the equivalence of equations 5.19, 5.20 to equations $5.1,5.2$

\subsection{Numerical Validation}

Numerical simulations were set up using MATLAB's one dimensional PDE solver pdepe in search for solutions to equation 5.5, following the numerical modeling by [81]. A constant depth mesh of $1 \mathrm{~mm}$ was used. The upper boundary condition was set as will be discussed, and a lower boundary condition of constant temperature was set at $z=10 \mathrm{~m}$, a large enough distance to not disturb the behaviour of the solution where the temperature measurements were analysed. An 
absolute tolerance of $10^{-6}{ }^{\circ} \mathrm{C}$ and a relative tolerance of $10^{-9}$ were used and an initial isothermal condition.

We ensure the simulation with the above mesh parameters yields reliable results by simulating soil thermodynamics with constant parameters and ensuring the simulation matches the exact solution to within $0.1 \%$. An example of the temperature field of such a simulation is given in Figure 5.5 .

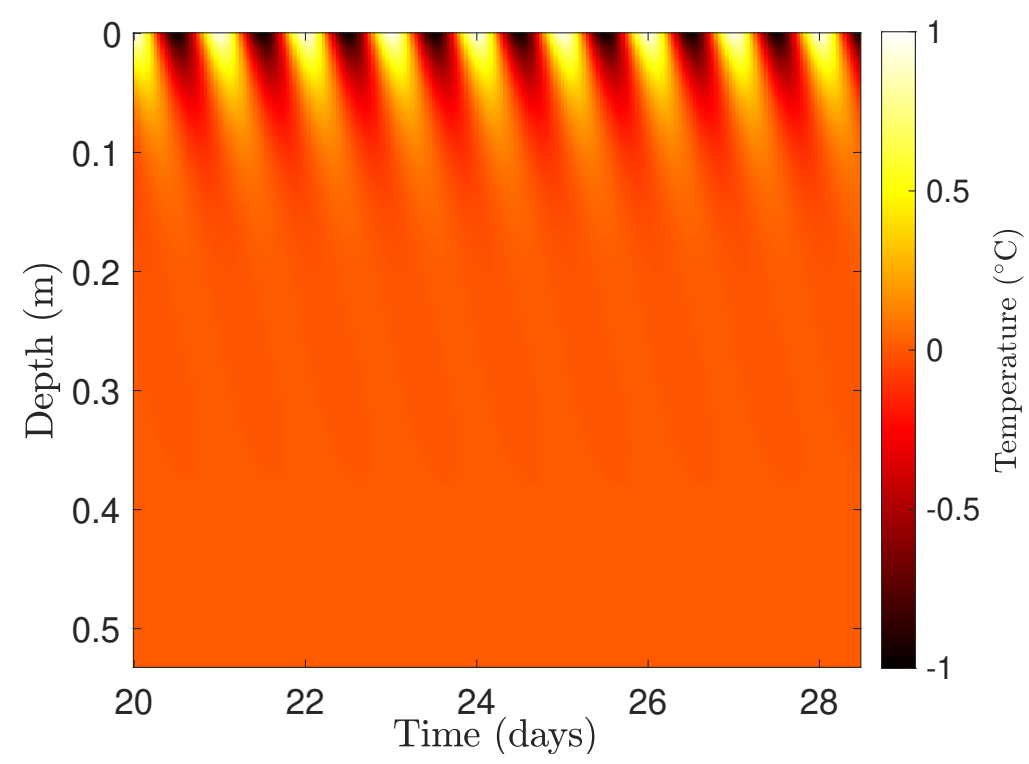

Figure 5.5: The oscillatory temperature field, shown in colour via the colourbar from -1 ${ }^{\circ} \mathrm{C}$ to $+1{ }^{\circ} \mathrm{C}$, simulated with a surface oscillation of amplitude $1{ }^{\circ} \mathrm{C}$ and periodicity of $1 /$ day.

We present two examples of numerical comparisons in Figure 5.6, one of a varying thermal conductivity and one for a varying seepage velocity. Soil temperatures were simulated with an oscillatory boundary condition of amplitude 1 ${ }^{\circ} \mathrm{C}$. The resulting thermal waves (for example, as seen in Figure 5.5) were analysed with sine waves being fit to the temperature series windowed to intervals of 1 oscillation for each mesh depth between $z=0$ and $z=0.1$ meters. The leading order WKB approximation is calculated from equation 5.16, with numerical integration being calculated using MATLAB's cumtrapz function over the selected mesh.

In Figure 5.6 we see very good agreement between the thermal wave propagation (both amplitude and phase) between the numerical simulations and the solutions to equation 5.16 in both comparisons. The largest errors occur for the lowest frequency wave of the varying thermal conductivity. We interpret this being due to the WKB approximation being an asymptotic relation valid in the 

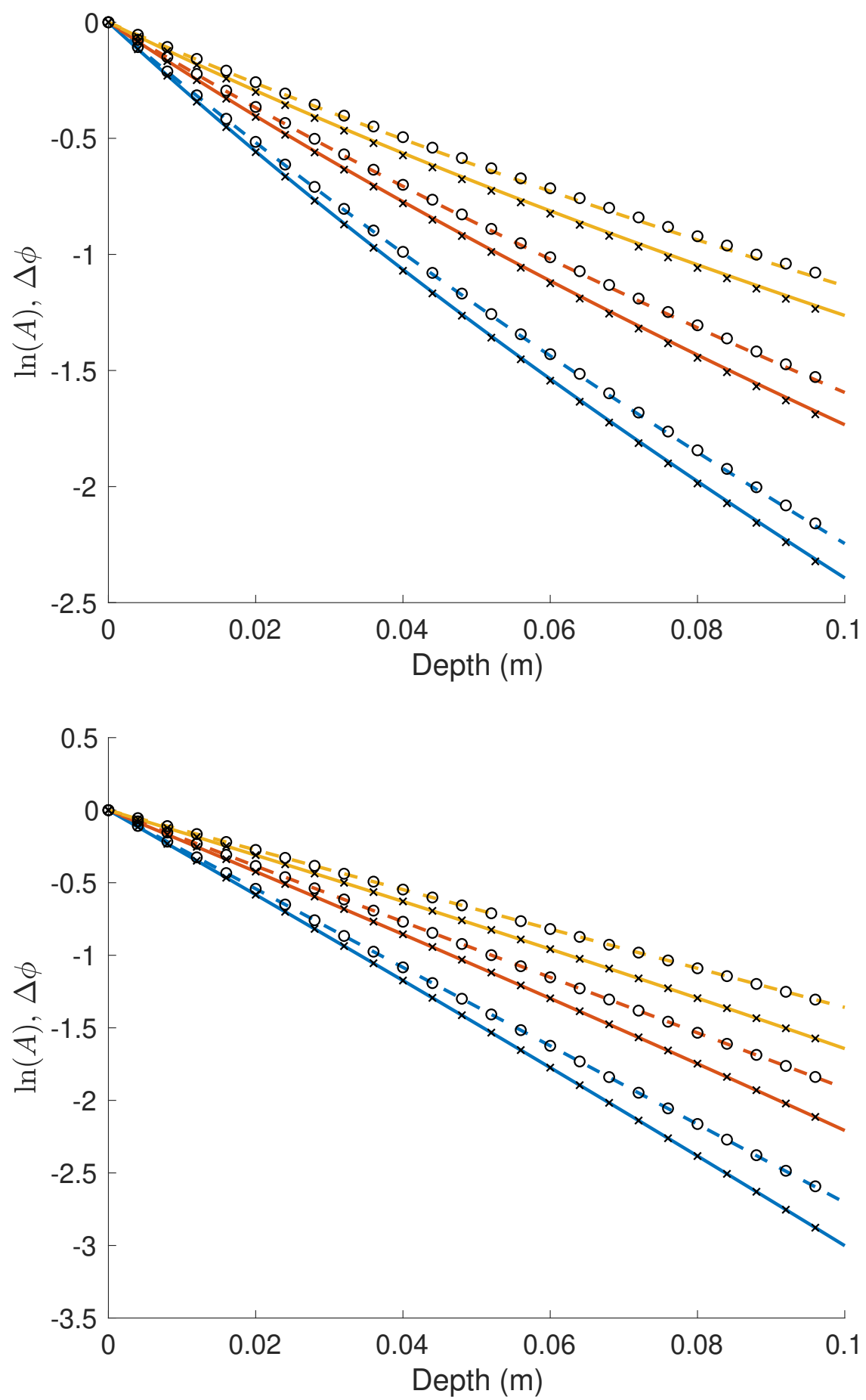

Figure 5.6: Amplitude decay (solid lines, $x$ 's) and phase shifts (dashed lines, o's) with a forced thermal wave at the upper boundary of frequency 0.5/day (yellow), 1/day (red) and 2/day (blue). The leading order WKB approximation (equation 5.16) is shown with the lines, overlaid with the numerical simulation results, as described in section 5.3, as symbols. A seepage velocity of $-10^{-6} \mathrm{~m} / \mathrm{s}$ and diffusivity of $10^{-7} \mathrm{~m}^{2} / \mathrm{s}$ were used at the upper boundary. Top: Thermal conductivity varies linearly and doubles over the range $z=0$ to $z=0.1 \mathrm{~m}$ with all other parameters held constant. Bottom: seepage velocity varies linearly with depth and doubles over the range $z=0$ to $z=0.1 \mathrm{~m}$ with all other parameter held constant. 
limit $\delta=D(0) / \omega \rightarrow 0$, and hence we expect larger errors to be present for lower thermal wave frequencies. 


\subsection{In-Homogeneous Soils with No Seepage}

In the case of no advective heat transport, we set the seepage velocity to zero to get $\xi=-k^{\prime}(z) / k(z)$ and $Q(z)=\xi^{2}-\xi^{\prime}$. For an analysis of diurnal signals $\left(\omega \approx 7 \times 10^{-5} \mathrm{~s}^{-1}\right)$ and a soil diffusivity of $D \approx 10^{-7} \mathrm{~m}^{2} / \mathrm{s}$ we have $\delta \approx 10^{-3}$. Assuming that $Q(z) \approx 1$ we see that $Q(z) \ll 1 / \delta \approx 100$ and so $Q(z)$ enters only in the third order WKB term. The leading order WKB terms of equation 5.15 are calculated as

$$
\begin{aligned}
& S_{0}=-\int_{0}^{z} \sqrt{\frac{i}{f(x)}} d x, \\
& S_{1}=-\frac{1}{4} \ln \left(\frac{i}{f(z)}\right)+\frac{1}{4} \ln \left(\frac{i}{f(0)}\right) .
\end{aligned}
$$

On the other hand, for a frequency of 1 /year (the annual cycle), such as in [76, 116 , we have $\omega \approx 2 \times 10^{-7} \mathrm{~s}^{-1}$ and $\delta \approx 0.5$. The term $\delta Q(z)$ may become comparable to the term $i / f(z)$ and the above approximation fails significantly. This would be evident if the measured behaviours conflict with the theory presented here or of [37]. For example, one might notice the amplitude decay and phase shift between two thermistors as a function of $\omega$ differing for low $\omega$. These are expected to be equal for a uniform soil or in the limit of high frequency thermal waves but expected to differ for lower frequency waves where $\delta Q(z)$ becomes comparable to $i / f(z)$.

Solving the associated equation for $U(z)$ (equation 5.14 gives $U(z)=\sqrt{k(0) / k(z)}$. Combining with the leading order WKB approximation of equation 5.15 gives the leading order behaviour of thermal waves in a soil with no advective heat transport as

$$
\theta_{n}(z) \sim \sqrt{\frac{k(0)}{k(z)}} f^{\frac{1}{4}}(z) \exp \left(-\frac{1}{\sqrt{\delta}} \int_{0}^{z} \sqrt{\frac{i}{f(x)}} d x\right), \delta \rightarrow 0
$$

The next term in the WKB series may be calculated and shown to be sufficiently small for $\delta<10^{-2}$. We compare this result to that of [37]. The leading order phase $(\phi)$ and amplitude $(r)$ are

$$
\begin{aligned}
& \phi=-\int_{0}^{z} \sqrt{\frac{1}{2 \delta f(x)}} d x, \\
& r=\sqrt[4]{f(z)} \sqrt{\frac{k(0)}{k(z)}} e^{\phi(z)} .
\end{aligned}
$$


Plugging these in to the RHS of the equations 5.3 and 5.4 respectively we get the results of [37] to within terms of order $\sqrt{\delta}$.

$$
\begin{aligned}
\mathrm{RHS}_{1} \sim \frac{k^{\prime}(z)}{k(z)}+\sqrt{\delta} \frac{k(z)^{2}\left(4 f(z) f^{\prime \prime}(z)-3 f^{\prime}(z)^{2}\right)+4 f(z)^{2} k^{\prime}(z)^{2}-8 f(z)^{2} k(z) k^{\prime \prime}(z)}{8 \sqrt{2} f(z)^{3 / 2} k(z)^{2}}+O\left(\delta^{1}\right) \cdot(5.24) \\
\mathrm{RHS}_{2}=\frac{1}{\delta f(z)}=\frac{\omega}{D_{0} f(z)} .
\end{aligned}
$$

This is expected as the WKB approximation used is valid up to terms of order $\sqrt{\delta}$. Note that the second equation is satisfied exactly.

\subsection{In-Homogeneous Soils with Seepage}

Following the methods in the constant parameter case above, the amplitude decay (real component of exponent) and phase shift (imaginary component of the exponent) may be written explicitly from equation 5.16 using trigonometric identities. Writing the solution as $\theta_{n} \sim e^{a(z)+i b(z)}$, where $a(z)$ denotes the amplitude decay and $b(z)$ denotes the phase shift with

$$
\begin{aligned}
& a(z)=-\int_{0}^{z}\left[\frac{1}{\sqrt{2}}\left(\sqrt{\sqrt{Q(x)^{2}+\left(\frac{\omega}{f(x) D_{0}}\right)^{2}}+Q(x)}-\sqrt{2} \xi(x)\right)\right] d x \\
& -\frac{1}{4} \ln \sqrt{\left(\frac{\omega}{D_{0} f(z)}\right)^{2}+Q(z)^{2}}+\frac{1}{4} \ln \sqrt{\left(\frac{\omega}{D_{0}}\right)^{2}+Q(0)^{2}},
\end{aligned}
$$

and

$$
\begin{aligned}
b(z) & =-\int_{0}^{z}\left[\frac{1}{\sqrt{2}}\left(\sqrt{Q(x)^{2}+\left(\frac{\omega}{f(x) D_{0}}\right)^{2}}-Q(z)\right)\right] d x \\
& -\frac{1}{4} \tan ^{-1}\left(\frac{\omega}{D_{0} f(z) Q(z)}\right)+\frac{1}{4} \tan ^{-1}\left(\frac{\omega}{D_{0} Q(0)}\right) .
\end{aligned}
$$

For most functional forms of $f(z)$ and $Q(z)$ these integrals do not admit analytical solutions. We further note that writing the above leading order in terms of the complex square root allows for easier integral evaluation and analysis.

\subsubsection{Numerical Examples}

Solutions to equation 5.16 with various parameter functions are presented in Figure 5.7, along with solutions using constant, average parameter values. The wave 
propagation behaviour, especially the amplitude decay, is relatively sensitive to changes in $k$. Variations in the specific heat capacity also induce relatively significant wave propagation behaviour, similarly to the varying $k$ case, with larger discrepancies in the amplitude decay. The difference between a varying seepage velocity and an average seepage velocity appear to be negligible in the example presented.

\subsection{Case Study - Linear Thermal Conductivity}

Here we analyse the errors introduced by methods assuming a constant, average thermal conductivity for a soil compared to a linear in depth variation of thermal conductivity (the simplest depth variation possible) to develop tools for studies such as [86]. Consider the case of a linear in depth thermal conductivity profile: $k(z)=k_{0}(1+a z)$, with all other parameters assumed constant. The parameter functions are therefore given by: $f(z)=1+a z, \xi(z)=\frac{q c_{w} \rho_{w}-a k_{0}}{2 k_{0}(a z+1)}$ and $Q(z)=$ $\frac{q^{2} c_{w}^{2} \rho_{w}^{2}-a^{2} k_{0}^{2}}{4 k_{0}^{2}(a z+1)^{2}}$.

In the leading order behaviour, equation 5.16, we denote the integral the ' $S_{0}$ term' and the remainder of the terms the ' $S_{1}$ terms'. We consider the following for comparison: $k_{\text {ave }}=k_{0}\left(1+\frac{1}{2} a z\right), f_{\text {ave }}=1+\frac{1}{2} a z, \xi_{\text {ave }}=\frac{q c_{w} \rho_{w}}{2 k_{0}\left(1+\frac{1}{2} a z\right)}$ and $Q_{\text {ave }}=\left(\frac{q c_{w} \rho_{w}}{2 k_{0}\left(1+\frac{1}{2} a z\right)}\right)^{2}$ being the average, constant parameter approximation, modelling the soil as homogeneous. These are attained by averaging the parameters between $0 \mathrm{~m}$ and $z \mathrm{~m}$. Under this approximation thermal waves of the form of equation 5.17 are present, with the appropriate terms substituted in.

\subsection{1 $S_{1}$ terms}

The $S_{1}$ terms reduce to 0 for the average, constant parameter approximation, $S_{1, \text { ave }}=0$. Defining the quantity $\xi_{0}=\left(q c_{w} \rho_{w}\right) /\left(2 k_{0}\right)$, we get the following form for the $S_{1}$ terms.

$$
S_{1}=\frac{1}{4}\left(\ln \left(\xi_{0}^{2}-\frac{a^{2}}{4}+\frac{i \omega}{D_{0}}\right)-\ln \left(\frac{4 \xi_{0}^{2}-a^{2}+\frac{4 i \omega(a z+1)}{D_{0}}}{4(a z+1)^{2}}\right)\right) .
$$



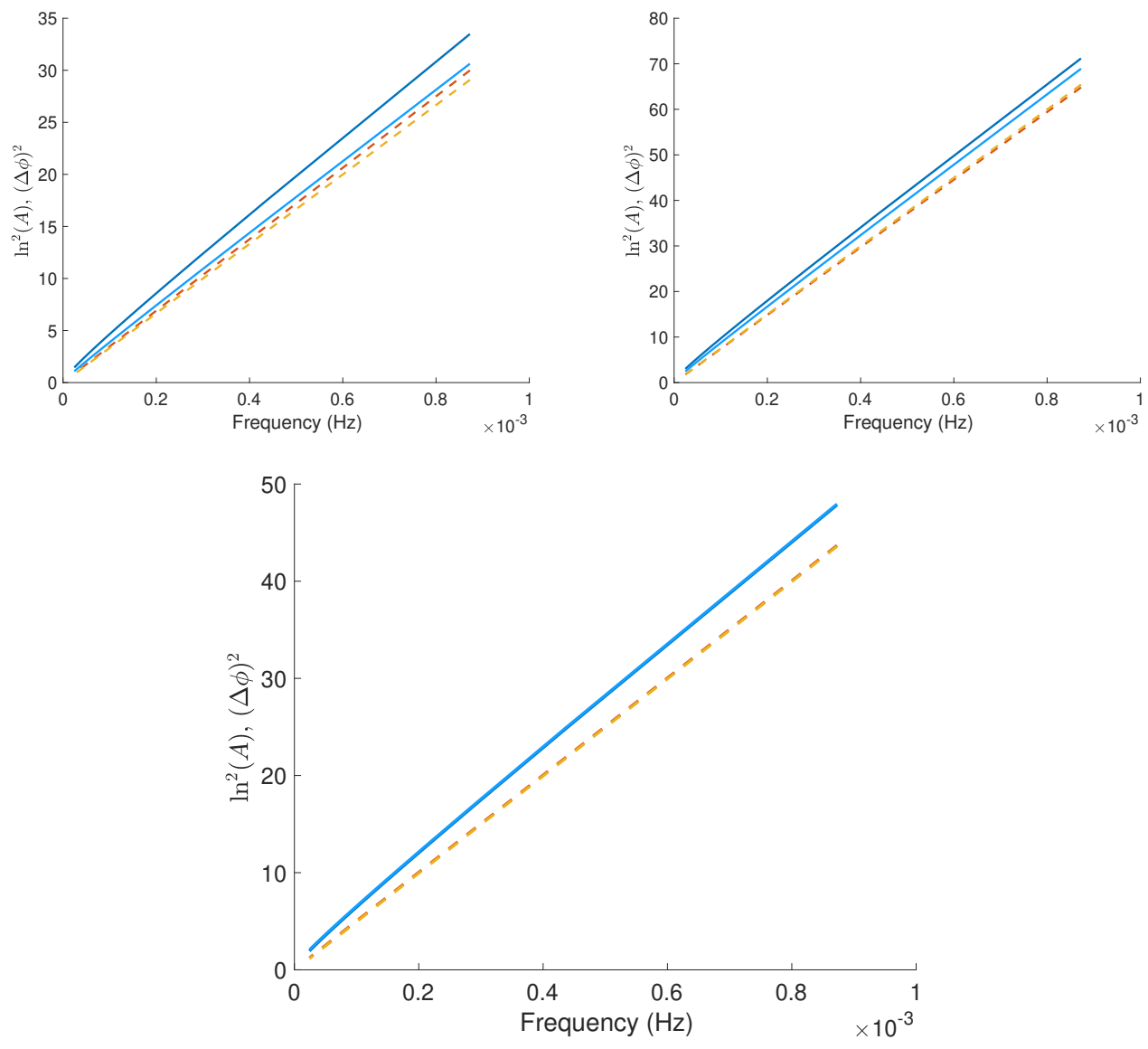

Figure 5.7: Amplitude decay (solid lines) and phase shift (dashed lines) of various thermal waves as a function of frequency, between a $0.1 \mathrm{~m}$ slab of soil. For each plot, two solutions to equation 5.16 are shown, the darker colours correspond to a soil with a linearly varying parameter and the lighter colours correspond to a soil with a constant, average value for that parameter. The varying parameters double over the $0.1 \mathrm{~m}$ depth and are Top Left: thermal conductivity, Top Right: specific heat capacity and Bottom: seepage velocity (the lines are so close they are practically indistinguishable for this plot). 
We consider three limits for instructive purposes: $\omega \rightarrow 0, \omega \rightarrow \infty$ and $a \rightarrow 0$. Each is given below

$$
\begin{array}{rlrl}
S_{1} & \sim \frac{1}{2} \ln (a z+1)+\frac{i a z \omega}{D_{0}\left(a^{2}-4 \xi_{0}^{2}\right)}+\mathcal{O}\left(\omega^{2}\right) & , \omega \rightarrow 0, \\
S_{1} \sim \frac{1}{4} \ln (a z+1)+\frac{i a z D_{0}\left(a^{2}-4 \xi_{0}^{2}\right)}{16 \omega(a z+1)}+\mathcal{O}\left(\frac{1}{\omega^{2}}\right) & , \omega \rightarrow \infty \\
S_{1} \sim \frac{a z\left(2 D_{0} \xi_{0}^{2}+i \omega\right)}{4 D_{0} \xi_{0}^{2}+4 i \omega}+\mathcal{O}\left(a^{2}\right) & , a \rightarrow 0 .
\end{array}
$$

We see that in both high and low frequencies, the term $\ln (1+a z)$ is present, with divergent behaviour as $1+a z$ tends to 0 . The term $D_{0}(1+a z)$ is the diffusivity at a depth $z$ which cannot reach zero, however, for a negative value of $a$, neglecting the term $\ln (a z+1)$ would introduce significant errors at depths. The first corrective terms in the high and low frequency limits are real and hence only affect the amplitude decay of the thermal wave. The second terms, proportional to $\omega$ and $\omega^{-1}$, are imaginary and so only affect the phase shift behaviour of the thermal waves. These are plotted along with the exact values of $S_{1}$ in Figure 5.8. This demonstrates that the amplitude and phase behaviour of the thermal waves are affected in different forms due to the depth dependence of the thermal conductivity.

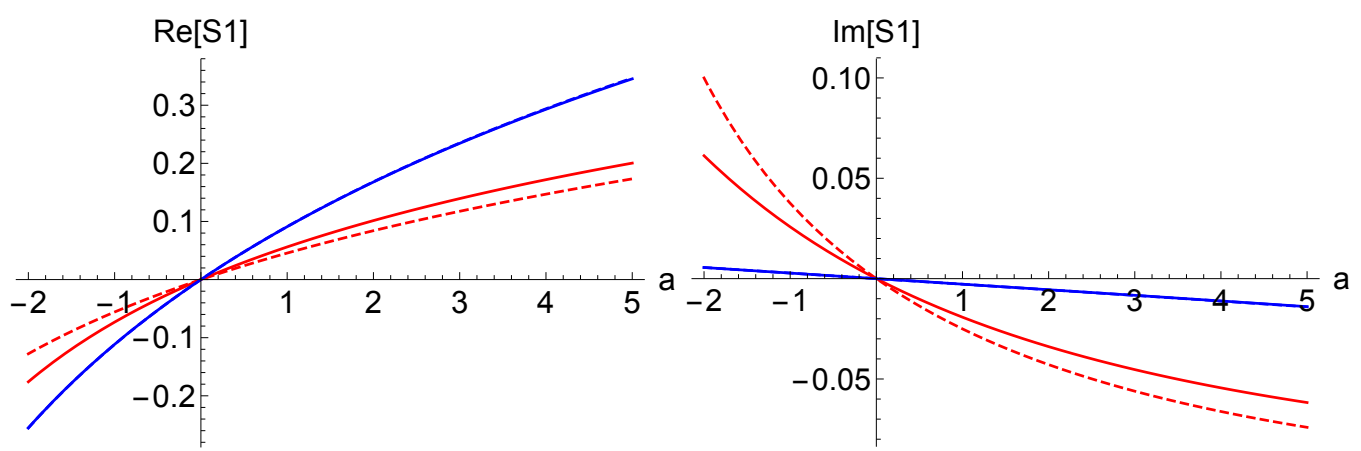

Figure 5.8: The real (left) and imaginary (right) parts of the term $S_{1}$ shown for a low frequency (1/month, solid blue line) and high frequency (1/day, soil red line) thermal wave. The corresponding low and high frequency limits given by equations 5.29 and 5.30 are plotted as dashed lines. The low frequency limit is in very good agreement with the exact value and can only just be distinguished in both plots. Parameters used are: $D_{0}=10^{-7} \mathrm{~m}^{2} / \mathrm{s}, q=-10^{-5} \mathrm{~m} / \mathrm{s}$ and $z=0.2 \mathrm{~m}$. 


\subsection{2 $S_{0}$ term}

For a thermal conductivity linearly varying in depth, the solution to $S_{0}$ may be written in terms of ln functions as below

$$
\begin{aligned}
S_{0}= & \frac{1}{2 a}\left(-2 \sqrt{\frac{\mathcal{C}}{D_{0}}}-\left(\sqrt{4 \xi_{0}^{2}-a^{2}}+a-2 \xi_{0}\right) \ln \left(\sqrt{-D_{0}\left(a^{2}-4 \xi_{0}^{2}\right)}-\sqrt{\mathcal{C}}\right)\right. \\
& \left.+\left(\sqrt{4 \xi_{0}^{2}-a^{2}}-a+2 \xi_{0}\right) \ln \left(\sqrt{-D_{0}\left(a^{2}-4 \xi_{0}^{2}\right)}+\sqrt{\mathcal{C}}\right)\right)\left.\right|_{x=0} ^{x=z},
\end{aligned}
$$

with $\xi_{0}=q c_{w} \rho_{w} /(2 k(0))$ and $\mathcal{C}=-a^{2} D_{0}+4 i a \omega x+4 D_{0} \xi_{0}^{2}+4 i \omega$. Using the average, constant parameters we get the following

$$
S_{0, \mathrm{ave}}=-\frac{z\left(\sqrt{4 D_{0} \xi_{0}^{2}+2 i \omega(a z+2)}-2 \sqrt{D_{0}} \xi_{0}\right)}{\sqrt{D_{0}}(a z+2)} .
$$

In order to gain further insight into the errors of the constant parameter assumption, we expand the error ratio of the average solution approximation below to leading order in $a$.

$$
\frac{S_{0}-S_{0, \text { ave }}}{S_{0}} \sim \frac{a}{2\left(\sqrt{\xi_{0}^{2}+i \frac{\omega}{D_{0}}}-\xi_{0}\right)}+\mathcal{O}\left(a^{2}\right) .
$$

It is evident that the error introduced is frequency dependent, and the complex values indicate that the amplitude and phase propagation behaviour are non-trivial. We expand the error in large and small frequencies to get the following.

$$
\begin{aligned}
& \frac{S_{0}-S_{0, \text { ave }}}{S_{0}} \sim 1+\frac{4 a z\left(\xi_{0}-\left|\xi_{0}\right|\right)}{\left(\sqrt{4 \xi_{0}^{2}-a^{2}}+a-2 \xi_{0}\right)(a z+2) \log (a z+1)}+\mathcal{O}(\omega) . \\
& \frac{S_{0}-S_{0, \text { ave }}}{S_{0}} \sim 1-\frac{a z}{\sqrt{2 a z+4}(\sqrt{a z+1}-1)}+\mathcal{O}\left(\frac{1}{\sqrt{\omega}}\right) .
\end{aligned}
$$

The two terms are plotted for a particular set of parameters against the value of $a$ in Figure 5.9. There is a trend of increasing error for larger absolute value of $a$, however, the complex structure of the error yields a curious dip around $a \approx 2 \xi_{0}$ in the low frequency limit. Further study of this structure is beyond the scope of our work. From this and the above equations it is evident that assuming a homogeneous thermal conductivity incurs significant wave propagation behaviour errors that depend on the amount of macroscale heterogeneity and the frequency of the thermal wave of interest. 


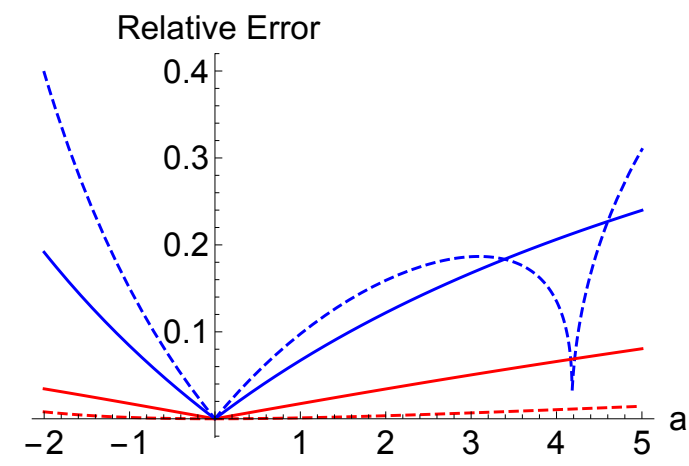

Figure 5.9: Absolute value of $\frac{S_{0}-S_{0, \text { ave }}}{S_{0}}$, being the relative error associated with an average thermal conductivity for a linear thermal conductivity for the $S_{0}$ term. Low and high frequency limits (equations 5.35 and 5.36) are shown in dashed lines (blue - high frequency limit, red - Low frequency limit). The exact error ratio is shown in solid lines for thermal waves of frequency $1 /$ day (red) and $1 /$ month (blue). Parameters used are: $D_{0}=10^{-7}$ $\mathrm{m}^{2} / \mathrm{s}, q=-10^{-6} \mathrm{~m} / \mathrm{s}$ and $z=0.2 \mathrm{~m}$.

\subsubsection{Errors in $\kappa_{e \text {,measured }}$ and $\xi_{\text {measured }}$}

Applying equations 5.19 and 5.20 onto a thermal wave of the form of equation 5.18, $\theta_{\text {hom }}$ (assumed to be a homogeneous soil with constant, uniform seepage velocity), with an average thermal conductivity, we get

$$
\begin{aligned}
\kappa_{\text {ave,measured }} & =D_{0}\left(1+\frac{a z}{2}\right), \\
\xi_{\text {ave,measured }} & =\frac{\xi_{0}}{1+\frac{a z}{2}}=\xi_{0}\left(1-\frac{a z}{2}\right)+\mathcal{O}\left(a^{2}\right) .
\end{aligned}
$$

These are exactly the average diffusivity and $\xi$ as we would expect. However, using the above WKB leading order behaviours we get the following, taken in the limits $a \rightarrow 0$ and $\frac{D_{0}}{\omega} \rightarrow 0$.

$$
\begin{aligned}
& \kappa_{\text {measured }}=D_{0}\left(1+\frac{a z}{2}\right)+a \frac{D_{0}^{2} \xi_{0}}{2 \omega}+\mathcal{O}\left(a^{2}+a \frac{D_{0}^{\frac{5}{2}} \xi_{0}^{2}}{\omega^{\frac{3}{2}}}\right) . \\
& \xi_{\text {measured }}=\xi_{0}\left(1-\frac{a z}{2}\right)-\frac{a}{4}-a \frac{\sqrt{D_{0}} \xi_{0}}{2 \sqrt{2 \omega}}+\mathcal{O}\left(a^{2}+\frac{D_{0} \xi_{0}^{2}}{\omega}\right) .
\end{aligned}
$$

The difference between $\kappa_{\text {measured }}$ and $D(z)$ is small as for most soil parameters of interest, we have $\frac{D_{0} \xi_{0}}{\omega} \ll D_{0}$. However the term $\frac{D_{0} \xi_{0}^{2}}{\omega}$ may not be smaller than $\xi_{0}$ 
for parameters of interest but only for relatively low seepage velocities. Expanding the full solution for $\xi_{\text {measured }}$, using the WKB approximation, to first order in $a$ we obtain

$$
\begin{aligned}
\xi_{\text {measured }}= & \xi_{0}\left(1-\frac{1}{2} a z\right)-\frac{1}{2} a-\frac{a \psi}{\sqrt{2}(\chi \sqrt{\psi-1}+\sqrt{\psi+1}) \operatorname{sgn}\left(\xi_{0}\right)-2 \psi} \\
- & \frac{1}{2} a\left(\psi^{2}\left(\sqrt{2}\left|\xi_{0}\right| \sqrt{\psi+1}-2 \xi_{0}\right)^{2}\right)^{-1} \\
\times & {\left[\sqrt{2} \xi_{0}\left|\xi_{0}\right|(2 \sqrt{\psi+1}+\chi(\sqrt{\psi-1}+\chi \sqrt{\psi+1}))\right.} \\
& \left.-\xi_{0}^{2}\left((\psi+2) \chi^{2}+2(\psi+1)\right)\right]
\end{aligned}
$$

with $\chi=\omega /\left(D_{0} \xi_{0}^{2}\right)$ and $\psi=\sqrt{\chi^{2}+1}$. The average system (equations 5.37 and 5.38 captures only the first correction terms from equations 5.39 and 5.40, omitting terms of first order in $a$. The omitted term in the diffusivity is a $D_{0}^{2} \xi_{0} /(2 \omega)$ which, for most applications, would be relatively small compared with the term $D_{0}(1+a z / 2)$. The omitted term for the variable $\xi_{\text {measured }}$ is $-a / 4-a \sqrt{D_{0}} \xi_{0} /(2 \sqrt{2 \omega})$ which is not small, specifically the term $\frac{a}{4}$.

We verify these numerically. Figure 5.10 shows the application of equations 5.19 and 5.20 onto thermal waves with a varying thermal conductivity as a function of depth. Shown are the parameters for a constant, average thermal wave, the leading order WKB form of the thermal wave and numerical simulations (Section 5.3). Equations 5.41 and 5.39 were used for calculations of the leading order WKB thermal wave. The greater heterogeneity in the simulated thermal conductivity resulted in a greater departure from the numerical simulation results under a homogeneous thermal wave assumption, whereas the leading order WKB remained in good agreement with the numerical simulations.

The example shown in Figure 5.10 shows the error of the average, constant thermal conductivity assumption to be about $6 \%$ and $2.5 \%$ for a thermal conductivity that increases by $5 \%$ and $2.5 \%$ over a depth of $0.1 \mathrm{~m}$, respectively. Measurements of thermal conductivity by [86] indicate that the thermal conductivity of various soils and sediments can vary between 0.55 and $2.96 \mathrm{~W} /(\mathrm{m} \mathrm{K})$ over a depth of $0.5 \mathrm{~m}$, corresponding to an average value of $a=1.08$. Hence we would expect much larger errors to be introduced in the constant thermal conductivity assumption for such a soil. This indicates that more careful attention should be given to the depth variation of the thermal conductivity when estimating low seepage velocities. 

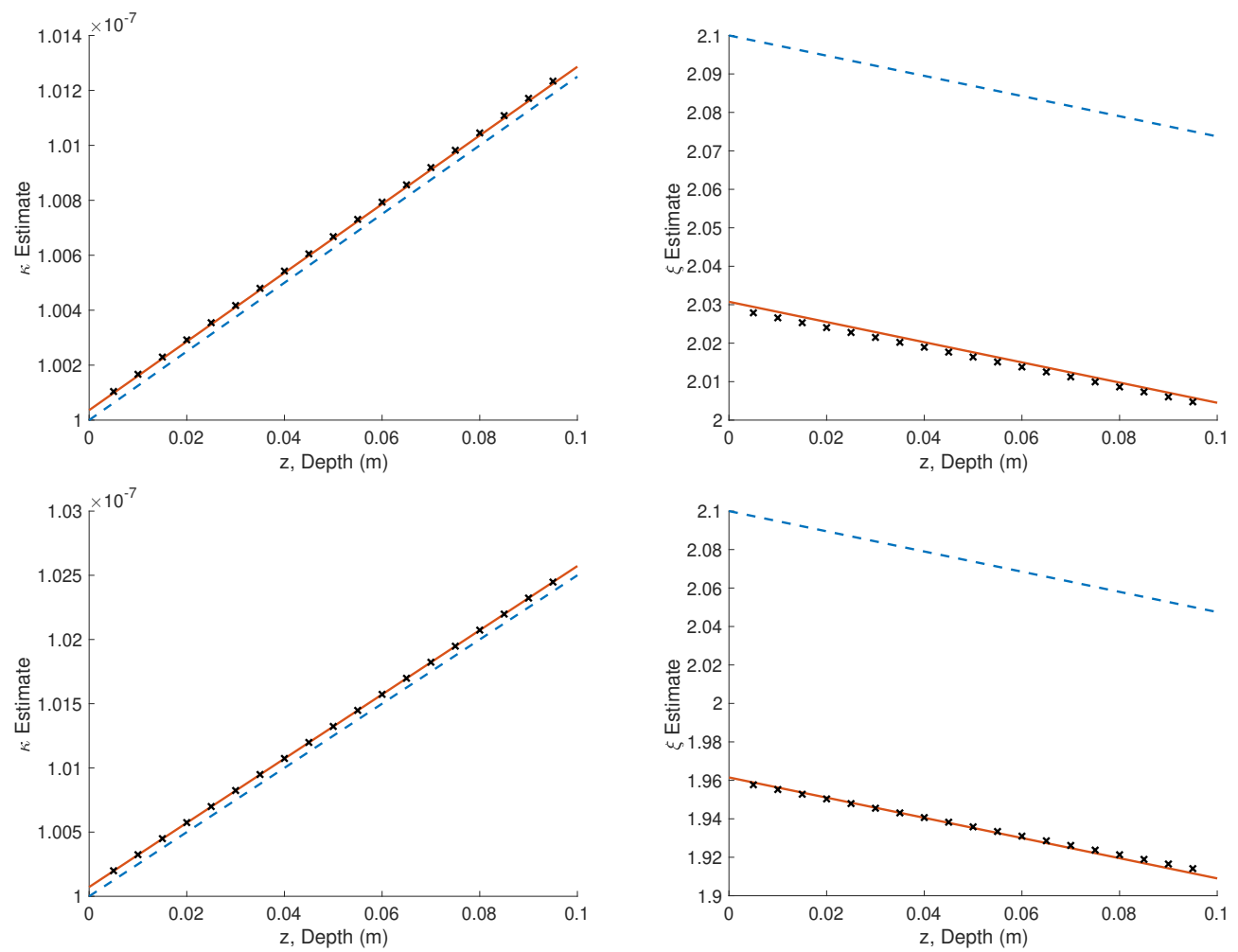

Figure 5.10: The estimate for $\kappa_{\text {measured }}$ (left) and $\xi_{\text {measured }}$ (right) from equations 5.19 and 5.20 for various approximations of a thermal waves with a linear thermal conductivity with depth gradient $a=0.25$ (top) and $a=0.5$ (bottom). The lines shown are for $a$ thermal wave with average, constant parameters (dashed blue), the leading order WKB estimate (solid red) and numerical simulations (black $x$ 's). Simulation parameters were: $k(0)=1 \mathrm{~W} /(\mathrm{m} \mathrm{K}), D_{0}=10^{-7} \mathrm{~m}^{2} / \mathrm{s}$ and $q=10^{-6} \mathrm{~m} / \mathrm{s}$. 


\section{Chapter 6}

\section{Conclusions}

Thermal wave behaviour was studied in depth dependent media with applications to sea ice and soils. We derive new and more accurate formulae for the amplitude and phase of thermal waves in sea ice and soils. These formulae are potentially useful for predicting sea ice and soil temperatures, inferring macroscale thermal properties of sea ice and soils, or for inferring vertical water velocities in soils.

A one-dimensional numerical solver was developed for sea ice thermodynamics. The sea ice growth rate and temperature field are solved for simultaneously using the latest parameterizations in the literature of the atmospheric heat fluxes, solar radiation absorption and thermal parameters of sea ice. Comparisons with numerous thermistor string measurements from the Arctic and Antarctic indicate simulations are accurate to within $1{ }^{\circ} \mathrm{C}$ during times of conduction-dominated heat transport. Periods of non-conductive heat transport (sea ice melt), which are not modelled, were seen to produce large discrepancies between the model and the measurements and hence may be easily identified. The numerical tool is designed to be easy to apply and the code and documentation are available on GitHub [8].

Following [66] we study the thermal waves generated by the absorption of solar radiation in sea ice. We further consider thermal waves due to oscillating air temperatures. Travelling thermal waves near the surface are attributed to the oscillating air temperatures and the quick decay of the solar heating term, whereas stationary thermal waves deeper in the sea ice are associated with solar heating. A differential equation is derived in the limit of high frequency, small amplitude thermal waves. Numerical solutions are shown to posses the same structure as the thermal waves present in thermistor string measurements. We use matched asymptotics and the WKB expansion to derive analytical expressions for the ther- 
mal waves. A criterion for travelling thermal waves to be present is derived using the inner and outer solutions. The leading order WKB approximation for the thermal waves is derived. The method of steepest descent is used to further approximate this in terms of elementary functions. Comparisons with numerical solutions of the differential equation and thermal waves from the numerical simulator indicate excellent agreement. Use of analytical expressions in determining the temperature field within sea ice would be of interest in developing lightweight simulators for sea ice temperature, an essential component of global climate simulations.

Using the same methods as for sea ice, soil thermodynamics are studied with emphasis on analysis of the diurnal thermal waves. As with sea ice, two time analysis is used to derive the leading order behaviour of high frequency, small amplitude thermal waves. A WKB approximation is used to leading order, giving a uniform approximation for the temperature oscillations, valid for depthvarying soils. This is shown to reduce to the Stallman equations under the appropriate conditions and agrees with the implicit results of [37]. The WKB approximation is compared with numerical simulations for a variety of parameters. The close agreement indicates the computationally cheap WKB approximation could serve as a tool for predicting soil temperatures in large scale simulations for depth-dependent soils without compromising significant accuracy.

Using the leading order WKB expansion we find that for soils with a depth dependent thermal conductivity, the tools in the literature give reliable estimates of the thermal diffusivity of the soil but significant systematic error in the seepage velocity. This is supported with a number of numerical examples. The analytical expressions developed would be of interest both in developing new tools for soil thermodynamics analysis and in understanding the limitations of the tools commonly used in the literature. We highlight the need for a variety of measurements to be undertaken in order for robust conclusions to be drawn.

Recent developments in soil thermodynamics include multi-frequency analysis [7]. The presented theory predicts frequency dependent thermal wave behaviour that may be scrutinised with accurate, high temporal resolution measurements. With further comparison between measurements, simulations and the presented theory, multi-frequency techniques could prove to give insight into the heterogeneity of the soil.

A significant assumption in the presented methods is the constant seepage velocity. It has been shown that such an assumption introduces significant errors in transient seepage velocity scenarios [81]. Further temperature measurements 
complemented by seepage measurements will give insight into the effects of transient seepage on the heat balance. Analytical studies of the effects of a transient seepage velocity on the thermal wave behaviour would be of great interest and applicability to better interpret such measurements, bound errors on seepage estimates and gain a deeper understanding of the heat transfer in transient seepage scenarios. 


\section{Bibliography}

[1] Ackley, S. F., Lewis, M. J., Fritsen, C. H., AND XIE, H. Internal melting in antarctic sea ice: Development of "gap layers". Geophysical Research Letters 35, 11 (2008).

[2] ANDERson, M. P. Heat as a ground water tracer. Groundwater 43, 6 (2005), 951-968.

[3] Andreas, E. L., Horst, T. W., Grachev, A. A., Persson, P. O. G., FAIRALL, C. W., GUEST, P. S., AND JORDAN, R. E. Parametrizing turbulent exchange over summer sea ice and the marginal ice zone. Quarterly Journal of the Royal Meteorological Society 136, 649 (2010), 927-943.

[4] Andreas, E. L., Persson, P. O. G., Grachev, A. A., Jordan, R. E., Horst, T. W., Guest, P. S., AND FAIRALL, C. W. Parameterizing Turbulent Exchange over Sea Ice in Winter. Journal of Hydrometeorology 11, 1 (02 2010), 87-104.

[5] Asllanaj, F., Jeandel, G., Roche, J. R., and Lacroix, D. Transient combined radiation and conduction heat transfer in fibrous media with temperature and flux boundary conditions. International Journal of Thermal Sciences 43, 10 (2004), 939 - 950.

[6] BEARDSMORE, G. Carrapateena TLOG.csv. https://melbourne. figshare.com/articles/dataset/Carrapateena_TLOG_CSV/ 6917351, 82018.

[7] Beardsmore, G., Egan, S., AND SANDiford, M. A fourier spectral method to measure the thermal diffusivity of soil. Geotechnical Testing Journal 43, 3 (Jul 2019), 565-587.

[8] Ben-Shachar, N. Sea ice growth modelling, matlab code. https:// doi.org/10.5281/zenodo.3883164. 
[9] Bender, C., And Orszag, S. Advanced Mathematical Methods for Scientists and Engineers: Asymptotic Methods and Perturbation Theory, vol. 1. Springer, New York, 011999.

[10] Bennett, Theodore J., J. A Coupled Atmosphere-Sea Ice Model Study of the Role of Sea Ice in Climatic Predictability. Journal of the Atmospheric Sciences 39, 7 (07 1982), 1456-1465.

[11] BRANDT, R. E., AND WARREN, S. G. Solar-heating rates and temperature profiles in antarctic snow and ice. Journal of Glaciology 39, 131 (1993), 99-110.

[12] Briggs, M. A., Lautz, L. K., McKenzie, J. M., Gordon, R. P., And HARE, D. K. Using high-resolution distributed temperature sensing to quantify spatial and temporal variability in vertical hyporheic flux. Water Resources Research 48, 2 (2012).

[13] Buntebarth, G., AND SCHOPPER, J. Experimental and theoretical investigations on the influence of fluids, solids and interactions between them on thermal properties of porous rocks. Physics and Chemistry of the Earth 23, 9 (1998), 1141 - 1146.

[14] Carslaw, H. S., And JAeger, J. C. Conduction of heat in solids, 2d ed. ed. Clarendon Press, Oxford, 1959.

[15] Cheng, B., Vihma, T., Rontu, L., Kontu, A., Pour, H. K., Duguay, C., AND PUlliainen, J. Evolution of snow and ice temperature, thickness and energy balance in lake orajärvi, northern finland. Tellus A: Dynamic Meteorology and Oceanography 66, 1 (2014), 21564.

[16] Comiso, J. C., AND Nishio, F. Trends in the sea ice cover using enhanced and compatible amsr-e, ssm/i, and smmr data. Journal of Geophysical Research: Oceans 113, C2 (2008).

[17] CRAnswick, R. H., COOK, P. G., Shanafield, M., And LAMOnTAGne, $S$. The vertical variability of hyporheic fluxes inferred from riverbed temperature data. Water Resources Research 50, 5 (2014), 3994-4010.

[18] DABbeRDT, W. F. A new model of thermal diffusion in a translucent medium. Boundary-Layer Meteorology 1, 3 (Jan 1971), 290-299.

[19] Dombrovsky, L. A., And Kokhanovsky, A. A. Solar heating of ice sheets containing gas bubbles. Journal of Quantitative Spectroscopy and $R a-$ diative Transfer 250 (2020), 106991. 
[20] EBERT, E. E., AND CURRY, J. A. An intermediate one-dimensional thermodynamic sea ice model for investigating ice-atmosphere interactions. Journal of Geophysical Research: Oceans 98, C6 (1993), 10085-10109.

[21] Feltham, D. L., Untersteiner, N., Wettlaufer, J. S., AND Worster, M. G. Sea ice is a mushy layer. Geophysical Research Letters 33, 14 (2006).

[22] Freitag, E., AND Busam, R. Complex Analysis. Universitext (1979). Springer, 2005.

[23] Golden, K. M., Ackley, S. F., And Lytle, V. I. The percolation phase transition in sea ice. Science 282, 5397 (1998), 2238-2241.

[24] Gough, A. J., Mahoney, A. R., Langhorne, P. J., Williams, M. J., ROBINSON, N. J., AND HASKELL, T. G. Signatures of supercooling: Mcmurdo sound platelet ice. Journal of Glaciology 58, 207 (2012), 38-50.

[25] Gryanik, V. M., LÜpkes, C., GracheV, A., And Sidorenko, D. New Modified and Extended Stability Functions for the Stable Boundary Layer based on SHEBA and Parametrizations of Bulk Transfer Coefficients for Climate Models. Journal of the Atmospheric Sciences 77, 8 (07 2020), 2687-2716.

[26] Haines, E. M., Buckley, R. G., And Trodahl, H. J. Determination of the depth dependent scattering coefficient in sea ice. Journal of Geophysical Research: Oceans 102, C1 (1997), 1141-1151.

[27] Hanesiak, J. M., Barber, D. G., And Flato, G. M. Role of diurnal processes in the seasonal evolution of sea ice and its snow cover. Journal of Geophysical Research: Oceans 104, C6 (1999), 13593-13603.

[28] Hartnett, J. P., And LUIKOV, A., Eds. Analytical Heat Diffusion Theory. Academic Press, 1968.

[29] HASHIN, Z., AND SHTRIKMAN, S. A variational approach to the theory of the effective magnetic permeability of multiphase materials. Journal of Applied Physics 33, 10 (1962), 3125-3131.

[30] Hatch, C. E., Fisher, A. T., Revenaugh, J. S., Constantz, J., And RUEHL, C. Quantifying surface water-groundwater interactions using time series analysis of streambed thermal records: Method development. Water Resources Research 42, 10 (2006). 
[31] Holmes, M. H. Introduction to Perturbation Methods. Springer New York, 2013.

[32] Hoppmann, M., Nicolaus, M., Hunkeler, P. A., Heil, P., Behrens, L.-K., KÖNIG-LANGLO, G., AND GERDES, R. Seasonal evolution of an iceshelf influenced fast-ice regime, derived from an autonomous thermistor chain. Journal of Geophysical Research: Oceans 120, 3 (2015), 1703-1724.

[33] Hu, G., ZhaO, L., Wu, X., Li, R., Wu, T., XIE, C., QIAO, Y., SHI, J., AND CHENG, G. An analytical model for estimating soil temperature profiles on the qinghai-tibet plateau of china. Journal of Arid Land 8, 2 (Apr 2016), 232-240.

[34] HuAng, W., Cheng, B., Zhang, J., ZhANG, Z., Vihma, T., LI, Z., AND NIU, F. Modeling experiments on seasonal lake ice mass and energy balance in the qinghai-tibet plateau: a case study. Hydrology and Earth System Sciences 23, 4 (2019), 2173-2186.

[35] Hunke, E. C., Lipscomb, W. H., And Turner, A. K. Sea-ice models for climate study: retrospective and new directions. Journal of Glaciology 56, 200 (2010), 1162-1172.

[36] Hunke, E. C., Notz, D., Turner, A. K., And Vancoppenolle, M. The multiphase physics of sea ice: a review for model developers. The Cryosphere 5, 4 (2011), 989-1009.

[37] Hurley, S., AND WiltshiRe, R. Computing thermal diffusivity from soil temperature measurements. Computers E Geosciences 19, 3 (1993), 475 - 477.

[38] III, A. W., AND CostA, G. B. Solving second-order differential equations with variable coefficients. International Journal of Mathematical Education in Science and Technology 39, 2 (2008), 238-243.

[39] Ingebritsen, S. E., SANFORd, W. E., AND Neuzil, C. E. Groundwater in geologic processes, 2nd edition. Cambridge University Press, 2006.

[40] Ingersoll, L., Zobel, O., AND Ingersoll, A. Heat Conduction, with Engineering and Geological Applications. International series in pure and applied physics. McGraw-Hill Book Company, 1948. 
[41] Irvine, D. J., CRanswick, R. H., Simmons, C. T., Shanafield, M. A., AND LAUTZ, L. K. The effect of streambed heterogeneity on groundwatersurface water exchange fluxes inferred from temperature time series. Water Resources Research 51, 1 (2015), 198-212.

[42] Irvine, D. J., LAutz, L. K., Briggs, M. A., Gordon, R. P., ANd MCKenZIE, J. M. Experimental evaluation of the applicability of phase, amplitude, and combined methods to determine water flux and thermal diffusivity from temperature time series using vflux 2. Journal of Hydrology 531 (2015), $728-737$.

[43] ISHIKAWA, N., AND KOBAYASHI, S. On the internal melting phenomenon (puddle formation) in fast sea ice. east antarctica. Annals of Glaciology 6 (1985), 138-141.

[44] KeEry, J., Binley, A., CROOK, N., And Smith, J. W. Temporal and spatial variability of groundwater-surface water fluxes: Development and application of an analytical method using temperature time series. Journal of Hydrology 336, 1 (2007), 1 - 16.

[45] Kevorkian, J., AND COLE, J. D. Multiple Scale and Singular Perturbation Methods. Springer New York, 1996.

[46] Key, J. R., LiU, Y., AND STONE, R. S. Development and evaluation of surface shortwave flux parameterizations for use in sea-ice models. Annals of Glaciology 25 (1997), 33-37.

[47] Koblick, D. Vectorized solar azimuth and elevation estimation v1.1.0.0. https://www.mathworks.com/matlabcentral/fileexchange/ 23051-vectorized-solar-azimuth-and-elevation-estimation.

[48] Kurylyk, B. L., Irvine, D. J., Carey, S. K., Briggs, M. A., Werkema, D. D., AND BONHAM, M. Heat as a groundwater tracer in shallow and deep heterogeneous media: Analytical solution, spreadsheet tool, and field applications. Hydrological Processes 31, 14 (2017), 2648-2661.

[49] LANDAU, H. Heat conduction in a melting solid. Quarterly of Applied Mathematics 8 (1950), $81-94$.

[50] Langhorne, P. J., SQuire, V. A., Fox, C., And Haskell, T. G. Lifetime estimation for a land-fast ice sheet subjected to ocean swell. Annals of Glaciology 33 (2001), 333-338. 
[51] Launiainen, J., AND CHeng, B. Modelling of ice thermodynamics in natural water bodies. Cold Regions Science and Technology 27, 3 (1998), 153 178.

[52] LAUniAinen, J., AND CHENG, B. Modelling of ice thermodynamics in natural water bodies. Cold Regions Science and Technology 27, 3 (1998), 153-178.

[53] LetTAu, H. Improved models of thermal diffusion in the soil. Eos, Transactions American Geophysical Union 35, 1 (1954), 121-132.

[54] LiaO, Z., Cheng, B., ZhaO, J., Vihma, T., Jackson, K., YANG, Q., YANG, Y., ZHANG, L., LI, Z., QIU, Y., AND CHENG, X. Snow depth and ice thickness derived from simba ice mass balance buoy data using an automated algorithm. International Journal of Digital Earth 12, 8 (2019), 962-979.

[55] Light, B., Grenfell, T. C., And Perovich, D. K. Transmission and absorption of solar radiation by arctic sea ice during the melt season. Journal of Geophysical Research: Oceans 113, C3 (2008).

[56] Light, B., Maykut, G. A., And Grenfell, T. C. Effects of temperature on the microstructure of first-year arctic sea ice. Journal of Geophysical Research: Oceans 108, C2 (2003).

[57] Light, B., Maykut, G. A., And Grenfell, T. C. A two-dimensional monte carlo model of radiative transfer in sea ice. Journal of Geophysical Research: Oceans 108, C7 (2003).

[58] Light, B., Perovich, D. K., Webster, M. A., Polashenski, C., And DADIC, R. Optical properties of melting first-year arctic sea ice. Journal of Geophysical Research: Oceans 120, 11 (2015), 7657-7675.

[59] Liu, J., Zhang, Z., InOue, J., AND Horton, R. M. Evaluation of snow/ice albedo parameterizations and their impacts on sea ice simulations. International Journal of Climatology 27, 1 (2007), 81-91.

[60] LU, S., REN, T., AND HORTON, R. Estimating the components of apparent thermal conductivity of soils at various water contents and temperatures. Geoderma 376 (2020), 114530.

[61] LU, Y., ZHOU, M., AND WU, T. Validation of parameterizations for the surface turbulent fluxes over sea ice with chinare 2010 and sheba data. Polar Research 32 (Sep. 2013). 
[62] Luce, C. H., Tonina, D., Gariglio, F., And Applebee, R. Solutions for the diurnally forced advection-diffusion equation to estimate bulk fluid velocity and diffusivity in streambeds from temperature time series. Water Resources Research 49, 1 (2013), 488-506.

[63] Maykut, G. A., And Untersteiner, N. Some results from a timedependent thermodynamic model of sea ice. Journal of Geophysical Research (1896-1977) 76, 6 (1971), 1550-1575.

[64] Maykut, G. A., AND Untersteiner, N. Some results from a timedependent thermodynamic model of sea ice. Journal of Geophysical Research (1896-1977) 76, 6 (1971), 1550-1575.

[65] McCallum, A. M., Andersen, M. S., Rau, G. C., And Acworth, R. I. A 1-d analytical method for estimating surface water-groundwater interactions and effective thermal diffusivity using temperature time series. Water Resources Research 48, 11 (2012).

[66] McGuinness, M. J., LAndman, K. A., Trodahl, H. J., And PantojA, A. E. Solar radiative heating in first-year sea ice. Annals of Glaciology 33 (2001), 261-266.

[67] Montiel, F., AND SQuire, V. A. Modelling wave-induced sea ice breakup in the marginal ice zone. Proceedings. Mathematical, physical, and engineering sciences 473, 2206 (Oct 2017), 20170258-20170258.

[68] Munz, M., Oswald, S. E., AND SCHMidT, C. Analysis of riverbed temperatures to determine the geometry of subsurface water flow around instream geomorphological structures. Journal of Hydrology 539 (2016), 74 87.

[69] NiCKERSON, A. Modelling solar heating of sea ice. Master's thesis, Victoria University of Wellington, 2007.

[70] NIWA. Cliflo. https://cliflo.niwa.co.nz/.

[71] Perovich, D., Richter-Menge, J., And Polashenski, C. Observing and understanding climate change: Monitoring the mass balance, motion, and thickness of arctic sea ice, 2020.

[72] Perovich, D. K. Ultraviolet Radiation and the Optical Properties of Sea Ice and Snow. Springer Berlin Heidelberg, Berlin, Heidelberg, 2002, pp. 73-89. 
[73] Perovich, D. K. Light reflection and transmission by a temperate snow cover. Journal of Glaciology 53, 181 (2007), 201-210.

[74] Perovich, D. K., Richter-Menge, J. A., Jones, K. F., Light, B., Elder, B. C., Polashenski, C., Laroche, D., Markus, T., and Lindsay, R. Arctic sea-ice melt in 2008 and the role of solar heating. Annals of Glaciology 52, 57 (2011), 355-359.

[75] PRIngle, D. J. Thermal Conductivity of Sea Ice and Antarctic Permafrost. PhD thesis, Victoria University of Wellington, 2004.

[76] Pringle, D. J., Dickinson, W. W., Trodahl, H. J., And Pyne, A. R. Depth and seasonal variations in the thermal properties of antarctic dry valley permafrost from temperature time series analysis. Journal of Geophysical Research: Solid Earth 108, B10 (2003).

[77] Pringle, D. J., Eicken, H., Trodahl, H. J., And Backstrom, L. G. E. Thermal conductivity of landfast antarctic and arctic sea ice. Journal of Geophysical Research: Oceans 112, C4 (2007).

[78] Pringle, D. J., Trodahl, H. J., AND Haskell, T. G. Direct measurement of sea ice thermal conductivity: No surface reduction. Journal of Geophysical Research: Oceans 111, C5 (2006).

[79] RAJEEV, P., AND KODIKARA, J. Estimating apparent thermal diffusivity of soil using field temperature time series. Geomechanics and Geoengineering 11, 1 (2016), 28-46.

[80] Rau, G. C., Andersen, M. S., McCallum, A. M., Roshan, H., And ACWORTH, R. I. Heat as a tracer to quantify water flow in near-surface sediments. Earth-Science Reviews 129 (2014), 40 - 58.

[81] Rau, G. C., Cuthbert, M. O., McCallum, A. M., Halloran, L. J. S., AND ANDERSEN, M. S. Assessing the accuracy of 1-d analytical heat tracing for estimating near-surface sediment thermal diffusivity and water flux under transient conditions. Journal of Geophysical Research: Earth Surface 120, 8 (2015), 1551-1573.

[82] Rayner, N. A., Parker, D. E., Horton, E. B., Folland, C. K., Alexander, L. V., Rowell, D. P., Kent, E. C., And Kaplan, A. Global 
analyses of sea surface temperature, sea ice, and night marine air temperature since the late nineteenth century. Journal of Geophysical Research: Atmospheres 108, D14 (2003).

[83] SAEnZ, B. T., AND ARRIGO, K. R. Simulation of a sea ice ecosystem using a hybrid model for slush layer desalination. Journal of Geophysical Research: Oceans 117, C5 (2012).

[84] SAlehin, M., PACKMAN, A. I., AND PARAdis, M. Hyporheic exchange with heterogeneous streambeds: Laboratory experiments and modeling. Water Resources Research 40, 11 (2004).

[85] Schneidewind, U., VAn Berkel, M., Anibas, C., Vandersteen, G., Schmidt, C., Joris, I., Seuntjens, P., BAtelaAn, O., AND ZWART, H. J. Lpmle3: A novel 1-d approach to study water flow in streambeds using heat as a tracer. Water Resources Research 52, 8 (2016), 6596-6610.

[86] SEBOK, E., AND MÜLLER, S. The effect of sediment thermal conductivity on vertical groundwater flux estimates. Hydrology and Earth System Sciences 23, 8 (2019), 3305-3317.

[87] Shampine, L. F. Ode solvers and the method of lines. Numerical Methods for Partial Differential Equations 10, 6 (1994), 739-755.

[88] Shampine, L. F., And Reichelt, M. W. The matlab ode suite. SIAM Journal on Scientific Computing 18, 1 (1997), 1-22.

[89] SHAN, C., AND Bodvarsson, G. An analytical solution for estimating percolation rate by fitting temperature profiles in the vadose zone. Journal of Contaminant Hydrology 68, 1 (2004), 83 - 95.

[90] Shanafield, M., Hatch, C., AND POHLl, G. Uncertainty in thermal time series analysis estimates of streambed water flux. Water Resources Research 47, 3 (2011).

[91] SHINE, K. P. Parametrization of the shortwave flux over high albedo surfaces as a function of cloud thickness and surface albedo. Quarterly Journal of the Royal Meteorological Society 110, 465 (1984), 747-764.

[92] SladeK, J., SlADEK, V., AND ZHANG, C. Transient heat conduction analysis in functionally graded materials by the meshless local boundary integral equation method. Computational Materials Science 28, 3 (2003), 494 - 504. Twelfth International Workshop on Computational Mechanics of Materials. 
[93] Smerdon, J. E., Beltrami, H., Creelman, C., And Stevens, M. B. Characterizing land surface processes: A quantitative analysis using airground thermal orbits. Journal of Geophysical Research: Atmospheres 114, D15 (2009).

[94] Smith, I., Langhorne, P., Frew, R., Vennell, R., And Haskell, T. Sea ice growth rates near ice shelves. Cold Regions Science and Technology 83-84 (2012), $57-70$.

[95] Smith, I. J., LAnghorne, P. J., Haskell, T. G., Joe Trodahl, H., FreW, R., AND Ross Vennell, M. Platelet ice and the land-fast sea ice of mcmurdo sound, antarctica. Annals of Glaciology 33 (2001), 21-27.

[96] Stallman, R. W. Steady one-dimensional fluid flow in a semi-infinite porous medium with sinusoidal surface temperature. Journal of Geophysical Research (1896-1977) 70, 12 (1965), 2821-2827.

[97] Stocker, T., Qin, D., Plattner, G.-K., Tignor, M., Allen, S., Boschung, J., Nauels, A., XiA, Y., BeX, V., And (EDS.), P. M. Climate change 2013: The physical science basis. contribution of working group $i$ to the fifth assessment report of the intergovernmental panel on climate change. IPCC (2013).

[98] Sutradhar, A., Paulino, G. H., and Gray, L. Transient heat conduction in homogeneous and non-homogeneous materials by the laplace transform galerkin boundary element method. Engineering Analysis with Boundary Elements 26, 2 (2002), 119 - 132.

[99] Tedesco, L., Vichi, M., HaApala, J., And StipA, T. A dynamic biologically active layer for numerical studies of the sea ice ecosystem. Ocean Modelling 35, 1 (2010), 89 - 104.

[100] Timco, G., AND Frederking, R. A review of sea ice density. Cold Regions Science and Technology 24, 1 (1996), 1 - 6.

[101] TimofeEV, A. M. Simulation of radiative heating of snow and ice coating. Thermophysics and Aeromechanics 25, 5 (Sep 2018), 765-772.

[102] Trodahl, H., Buckley, R., And Vignaux, M. Anisotropic light radiance in and under sea ice. Cold Regions Science and Technology 16, 3 (1989), $305-308$. 
[103] Trodahl, H. J., McGuinness, M. J., LAnghorne, P. J., Collins, K., PANTOJA, A. E., SMith, I. J., AND HASKell, T. G. Heat transport in mcmurdo sound first-year fast ice. Journal of Geophysical Research: Oceans 105, C5 (2000), 11347-11358.

[104] Turner, K. E., Smith, I. J., Tison, J.-L., VerbeKe, V., McGuinness, M., INGHAM, M., VENNELL, R., AND TRODAHL, J. Sea ice growth rates from tide-driven visible banding. Journal of Geophysical Research: Oceans 122, 6 (2017), 4675-4684.

[105] Uttal, T., Curry, J. A., McPhee, M. G., Perovich, D. K., Moritz, R. E., Maslanik, J. A., Guest, P. S., Stern, H. L., Moore, J. A., Turenne, R., Heiberg, A., Serreze, M. C., Wylie, D. P., Persson, O. G., Paulson, C. A., Halle, C., Morison, J. H., Wheeler, P. A., Makshtas, A., Welch, H., Shupe, M. D., Intrieri, J. M., Stamnes, K., Lindsey, R. W., Pinkel, R., Pegau, W. S., Stanton, T. P., And GrenFELD, T. C. Surface Heat Budget of the Arctic Ocean. Bulletin of the American Meteorological Society 83, 2 (02 2002), 255-276.

[106] Vaughan, D. G., Marshall, G. J., Connolley, W. M., Parkinson, C., Mulvaney, R., Hodgson, D. A., King, J. C., Pudsey, C. J., And TURNER, J. Recent rapid regional climate warming on the antarctic peninsula. Climatic Change 60, 3 (Oct 2003), 243-274.

[107] Wang, C., Granskog, M. A., Gerland, S., Hudson, S. R., Perovich, D. K., Nicolaus, M., IVAn Karlsen, T., Fossan, K., And Bratrein, M. Autonomous observations of solar energy partitioning in first-year sea ice in the arctic basin. Journal of Geophysical Research: Oceans 119, 3 (2014), 2066-2080.

[108] WELLER, G. The effect of absorbed solar radiation on the thermal diffusion in antarctic fresh-water ice and sea ice. Journal of Glaciology 6, 48 (1967), 859-878.

[109] Wendler, G., MoOre, B., Dissing, D., And Kelley, J. On the radiation characteristics of antarctic sea ice. Atmosphere-Ocean 38, 2 (2000), 349-366.

[110] Winton, M. A Reformulated Three-Layer Sea Ice Model. Journal of Atmospheric and Oceanic Technology 17, 4 (04 2000), 525-531. 
[111] Wongran, P., Langhorne, P. J., Dempsey, D. E., Hahn-Woernle, L., AND SUN, Z. Simulation of the crystal growth of platelet sea ice with diffusive heat and mass transfer. Annals of Glaciology 56, 69 (2015), 127-136.

[112] WORSter, M., AND WetTlaufer, J. Natural convection, solute trapping and channel formation during solidification of salt water. Journal of Physical Chemistry B101 (1997), 6132 - 6136.

[113] WÖRman, A., Riml, J., Schmadel, N., Neilson, B. T., BotTACiNBusolin, A., AND HEAvilin, J. E. Spectral scaling of heat fluxes in streambed sediments. Geophysical Research Letters 39, 23 (2012).

[114] YANG, Y., LEPPÄRANTA, M., CHENG, B., AND LI, Z. Numerical modelling of snow and ice thicknesses in lake vanajavesi, finland. Tellus A: Dynamic Meteorology and Oceanography 64, 1 (2012), 17202.

[115] YEN, Y.-C. Review of thermal properties of snow, ice and sea ice. https: //apps.dtic.mil/dtic/tr/fulltext/u2/a103734.pdf, 061981.

[116] Zhu, D., Ciais, P., Krinner, G., Maignan, F., Jornet Puig, A., And Hugelius, G. Controls of soil organic matter on soil thermal dynamics in the northern high latitudes. Nature Communications 10, 1 (Jul 2019), 3172. 


\section{Appendix A}

\section{Physical Parameters and Constants}

Here we summarise parameterization of sea ice and snow properties from the literature. Temperature, $T$, is measured in degrees Celsius, and salinity, $S$, in units of grams per gram (note that this is a factor of 1000 smaller than other standard units such as ppt, parts per thousand). All other quantities are given in SI units.

\section{A.1 Ice Properties}

- Thermal Conductivity [77] [W/(m C)]:

$$
k_{\text {ice }}=\frac{\rho}{\rho_{\text {ice }}}\left[2.11-0.011 T+0.09 \frac{S}{T}-\frac{\rho-\rho_{\text {ice }}}{1000}\right] .
$$

- Heat Capacity [78, 115] [J/(kg C)]:

$$
\begin{aligned}
c_{\text {ice }}=1000(2.113+0.0075 T-0.0034 S \times 1000 & +0.00008 S T \times 1000 \\
& \left.+18.04 \frac{S \times 1000}{T^{2}}\right) .
\end{aligned}
$$

- Density [115] $\left[\mathrm{kg} /\left(\mathrm{m}^{3}\right)\right]$

$$
\rho_{\text {ice }}=\left(1-V_{a}\right)\left(1-\frac{4.51 S}{T}\right) 917 .
$$

- Latent Heat of Fusion [115] [J/kg]:

$$
L_{\text {ice }}=4184\left(79.68-0.505 T-27.3 S+4311.5 \frac{S}{T}\right) .
$$




\section{A.2 Snow Properties}

- Density [64] [kg/m³ ]: $\rho_{\text {snow }}=330$.

- Thermal Conductivity [115] [W/(m C)]:

$$
k_{\text {snow }}=0.0688 \exp \left(0.0088 T+4.6682 \frac{\rho_{\text {snow }}}{1000}\right) \text {. }
$$

- Heat Capacity [115] [J/(kg C)]:

$$
c_{\text {snow }}=(2.7442+0.1282(T+273.15)) \times \frac{18.02}{1000} .
$$

\section{A.3 Common Soil Properties}

- Volumetric Heat Capacity [13] [J/( $\left.\left.\left.\mathrm{C} \mathrm{m}^{3}\right)\right]\right]: ~ \rho c \approx 2-4 \times 10^{6}$.

- Thermal Conductivity [86] [W/(m C)]: $k \approx 0.55-2.96$.

- Water Seepage Velocity [17] [m/s]: $q \approx 8 \times 10^{-6}$. 